\title{
Bioengineering Abstracts
}

\section{Session - Tissue Engineering and Artificial Organs Room 2, Thursday 3 May, 09.00 - 10.30}

\section{Artificial myocardial tissue (AMT) - A novel contractile engineered heart muscle}

T. Kofidis, P.Akhyari, U.Martin, J.Boublik, P.Theodorou, A.Ruhparwar, T.Kraft, HP.Kubis, B.Brenner, G.Gros, A. Haverich

Objectives: With the advent of tissue engineering a new era in cardiovascular research has begun. In our search for therapeutic alternatives to human organ donation and xenotransplantation we developed a novel artificial myocardial tissue with unique properties and multiple applications.

Methods: We have harvested ventricles from 2 to 3 day old neonatal rats. After trypsinisation the obtained cell population was preplated then embedded in a collagen-Matrigel mixture and left to consolidate at $37^{\circ} \mathrm{C}$. HE stains and electron microscopy were performed to evaluate the microscopical structure of the artificial myocardial tissue. Protein and RNA analysis was carried out by means of 2Delectrophoresis, resp. RT-PCR. Force development dynamics were measured in a unique apparatus which allows for sensitive registrations.

Results: We obtained high and vital cell populations. Embedding proved efficient at a concentration of $2 \times 10^{6}$ cells / tissue unit $(1 \mathrm{~cm} \times 1 \mathrm{~cm})$. We achieved a reproducible, vital and macroscopically contracting artificial myocardial tissue which continued spontaneous, synchronised and ubiquitary activity for more than 12 weeks with minimal culture requirements. The observed contractions were not limited to the rare of the tissue but also occurred in the center equally in contrast to reports elsewhere. The mechanical stability is sufficient to allow for transfer and manipulation of the cardiac tissue unit with the tip of a forceps.
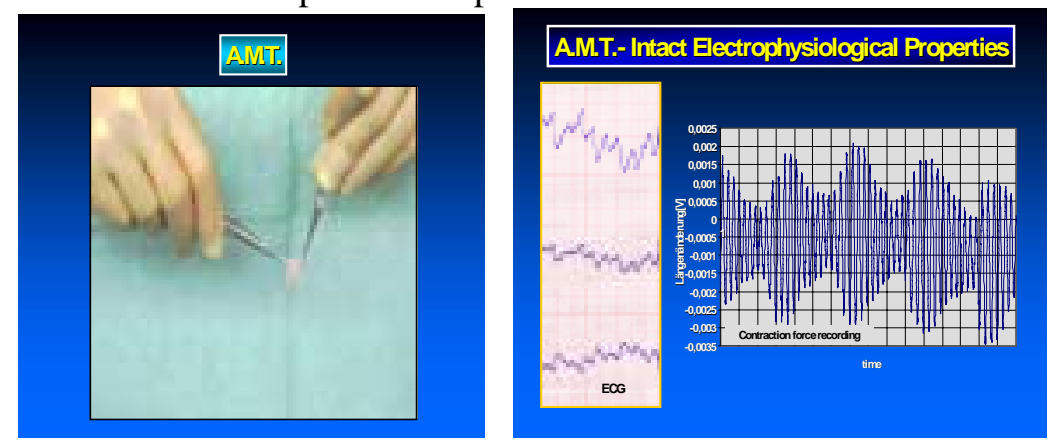
The microscopical structure shows intercalations between cells in a mesh like pattern. Protein analysis revealed actin, ventricular light chain 1 and 2 ( $\mathrm{vLC} 1$ and 2), and tropomyosin distribution equal to native cardiac tissue. When suspended in a force detector, artificial myocardial tissue developed measurable spontaneous actions but also responded to stretch, $\mathrm{Ca}$ and epinephrine administration with signal enhancement. Response on stretch resembled the Frank-Starling law of the Heart.

Conclusions: We were able to produce contractile myocardial tissue in vitro, using simple materials. Mechanical and biological properties of our engineered heart tissue resembles native cardiac tissue. We consider artificial myocardial tissue with these properties to be a powerfull tool for in vitro investigation (co-cultures, gene technological and pharmacological studies), cell transfer, tissue repair and organ fragment construction.

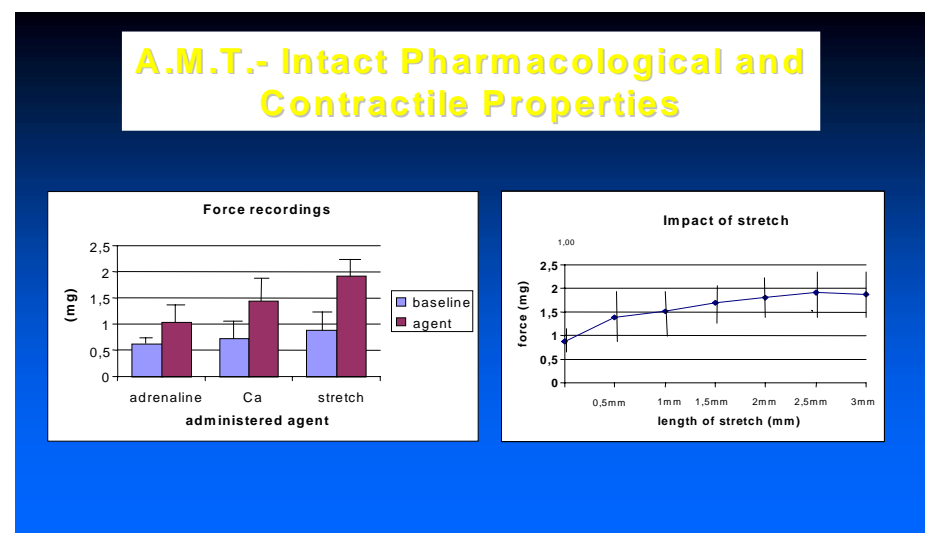

Development of pulsatile bioreactor for the in vitro formation of tissue engineered heart valves

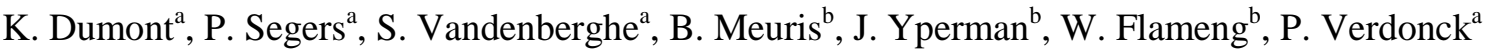 \\ ${ }^{a}$ IBITECH, Institute of Biomedical Technology, Ghent University, Belgium \\ ${ }^{b}$ CEHA, Centre for Experimental Heart Chirugy and Anaesthesiology, KUL, Belgium
}

Introduction:Valvular heart disease is an important cause of morbidity and mortality. Currently, homografts, mechanical valves and bioprostheses are used as substitutes for failing native valves. All of them have serious limitations including poor durability, partially due to calcification, thromboembolism and rejection. A new promising alternative to overcome these shortcomings is tissue engineering (TE) of heart valves. As tissue engineered constructs contain living cells, they have the potential for growth, repair and remodelling.

Basic Demands of the Bioreactor: The aim of this bioreactor is to create a physiological environment in which acellularised porcine and bovine aorta valves, seeded with human cells, can resident during a longer period (days, weeks). The bioreactor should be very compact to fit in an incubator and contain as little medium as possible. The use of a pump (heat production) may not disturb the climate in the incubator. The medium should be exposed to the controlled atmosphere (diffusion of $\mathrm{O}_{2}, \mathrm{CO}_{2}, \mathrm{~N}_{2}$ ) in the incubator and have good access to all sides of the valve. It should be 
possible to refresh (a part of) the medium in a simple way and to sterilise all parts. This way, a micro-environment is created with sufficient nutrients and without accumulation of metabolites. The bioreactor should allow testing entire valves as well as parts of it. The valve must be easily accessible for biopsy to check the progress of the cell growth. From haemodynamic point of view, the bioreactor should be flexible in controlling parameters such as pressure conditions, stroke volume and frequency. The whole system must be easy to start up and the parameters are required to remain stable for a period of a few days.

Bioreactor Design: The bioreactor consists of three major parts (Figure 1). The first main part (S) is the left ventricle (LV) made of silicone-rubber (Wilsor, the Netherlands). This LV is compressed and decompressed by the movement of a piston, which allows varying the stroke volume and the frequency. It is thus possible to create different pulsatile flow conditions. The second part is the compliance (C) that represents the elastic function of the large arteries. Between the LV (S) and the compliance (C) the TE aortic valve is placed (V). Distal to this compliance chamber is a resistance $(\mathrm{R})$, mimicking the resistance of the arterioles and capillaries. With controllable resistance, compliance, stroke volume and frequency, it is possible to change the hemodynamic conditions over a wide physiological range. The mechanical valve (M) ensures the flow in one direction.

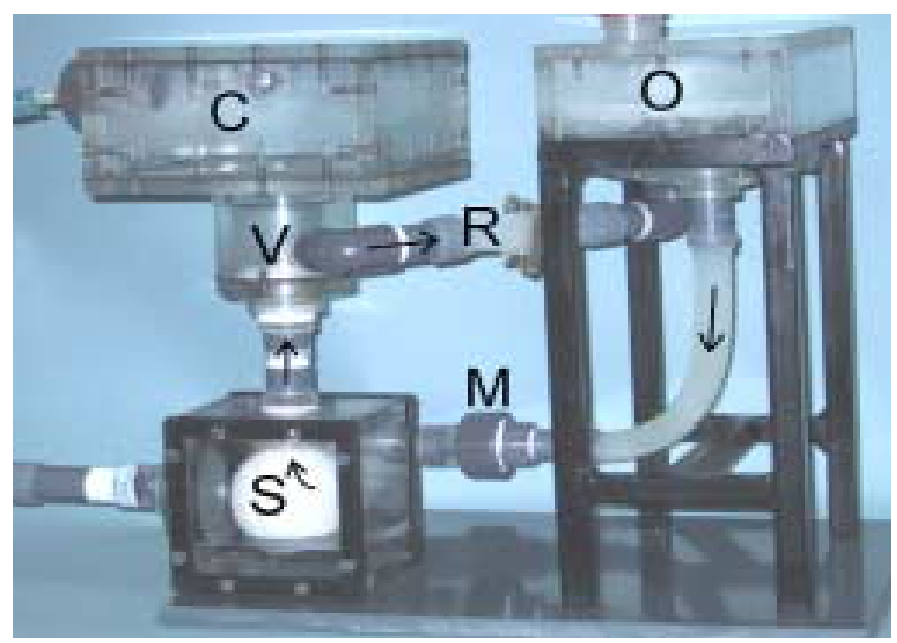

Fig. 1: Basic design of pulsatile bioreactor

C:Compliance; R:Resistance; O:Reservoir; V:TE valve;

M: Mechanical valve,A:Aerator; S:Left ventricle (silicone);

E:Extern circuit; P:Pressure transducer

Results and discussion: This study resulted in a prototype of a compact pulsatile flow system that fits in an incubator. Safe and easy handlng of the bioreactor is ensured by its simple design. Although the ideal conditions for the creation of tissue engineered heart valves are yet unknown, one can expect that exposure of devoloping tisue to physiological conditions might be beneficial [1]. Thus, the $\mathrm{n}$ vitro formation of a haemodynamically and mechanically competent tissue under simulated physiological conditions (Figure 2) can potentially be achieved with the bioreactor here described. Future research includes the numerical research of the pulsatile flow conditions, shear stress distribution and pressure distribution in the vicinity of the aortic valve. Thus, numerical 
simulation in combination with experimental studies in the bioreactor allows studying the impact of these mechanical aspects on the development of the seeded cells.

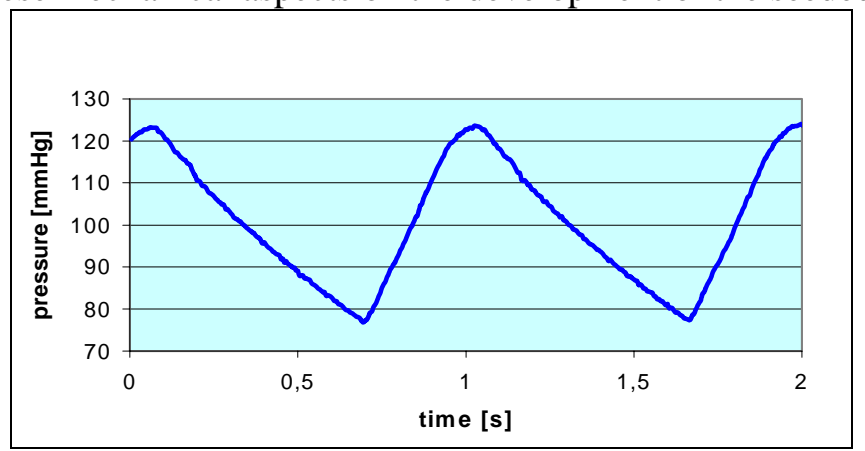

Fig. 2: Measured result of aortic pressure

\title{
Acknowledgement
}

This research is funded by a specialisation grant of the Flemish Institute for the Promotion of the Scientific-Technological Research in Industry (IWT).

\section{Reference}

[1] S. P. Hoerstrup, M.D., R. Sodian, M.D., J.S. Sperling, M.D., J.P. Vacanti, M.D., and J.E. Mayer,

Technical report: new pulsatile bioreactor for in vitro formation of tissue engineered heart valves. Tissue Engineering (Volume 6, Number 1, 2000)

\section{Endothelialization of cardiovascular grafts. Polydimethylsiloxane content affects adhesion and growth of endothelial cells onto polyurethane grafts}

\author{
G. Soldani, E. Briganti, P. Losi, I. Martinelli \\ Laboratory for Biomaterials \& Graft Technology, Institute of Clinical Physiology C.N.R. Massa, Italy
}

Introduction: It is well known that the replacement of arteries with purely synthetic vascular prostheses often leads to the failure of such reconstruction when small-diameter or low-flow configurations are concerned, due to the thombogenicity of the artificial blood contacting surface. One of the most promising approaches to improve long-term patency of these grafts is to coat the surface with endothelial cells (EC). This has been suggested because an endothelial cells monolayer provides a thromboresistant surface to circulating blood and because vascular grafts placed in humans do not or rarely spontaneously form an EC monolayer1.

The aim of this work was to develop new polyurethane (PU)-polydimethylsiloxane (PDMS) graft materials composition to enhance cell proliferation; in particular it was assessed the influence of PDMS content on the adherence and growth of seeded EC. It has been reported that EC don't adhere to bare materials so we pre-treated polymer grafts with fibronectin (Fn) to facilitate cellular adhesion onto polymer surfaces and to develop engineered vascular grafts with improved functionality in the long-term.

Materials and Methods: Sample preparation: The new elastomeric graft material (named Silcrothane) was prepared in our laboratory by reacting a standard PU with PDMS in different content $(10 \%, 20 \%$ and $30 \%)$. The Silcrothane material was used to realise vascular graft using a "spray, phase 
inversion" technique, that allows to control internal surface micro-porosity2. Low porosity surfaces were chosen because preliminary experiments showed that this kind of surfaces enhance cells adhesion and growth.

Grafts were sterilised by 20 ' of sonication in $\mathrm{HCl} 0.4 \mathrm{~N}$ and then they were rinsed in distilled sterile water to remove $\mathrm{HCl}$ solution.

Endothelial cell culture: Human umbilical vein endothelial cells (HUVEC) were harvested by treatment with $0,1 \%$ collagenase as described in literature3 and maintained in M-199 supplemented with $10 \%$ FBS. Experiments were carried out with cultures at passage earlier than fourth.

Adhesion and growth tests: The sterile grafts were cut into circular pieces of about $1 \mathrm{~cm} 2$ and they were fitted into 24-wells plates. The culture plastic served as control. Pieces and controls were incubated with Fn $(6 \mu \mathrm{g} /$ well $)$ for $1 \mathrm{~h}$ at $37^{\circ} \mathrm{C}$, other pieces and controls with PBS in the same conditions. Then $0.5 \mathrm{ml}$ HUVEC suspensions $(1 \times 105$ cells $/ \mathrm{ml})$ were seeded on them. After $18 \mathrm{~h}$ (adhesion test), 3, 5 and 7days (growth test) of incubation the number of attached cells was determined.

Qualitative evaluation of adhered EC was performed by light microscopy observation and compared with controls. The cells were stained with Giemsa ( $0.1 \%$ in methanol) after fixation with methanol $70 \%$. Metabolic activity of cells was tested using the tetrazolium-based colorimetric method (MTT test) to quantify cellular viability.

Results: Light microscopy observations of the bare and Fn-coated materials showed that cell adhesion was similar on every pieces and lesser than controls. Therefore treatment with Fn was found to not induce a significant improvement of cell adhesion on the polymer surfaces.

However, no growth of HUVEC was observed on every non-coated polymers. We observed Fncoating increased the HUVEC growth rate only on Silcrothane with 10\% PDMS (Fig.1); on this surface cells appeared to be completely spread, showing their characteristic morphology. On the contrary, on the other materials cells maintained a round shape. MTT test consolidated the microscopical observations.
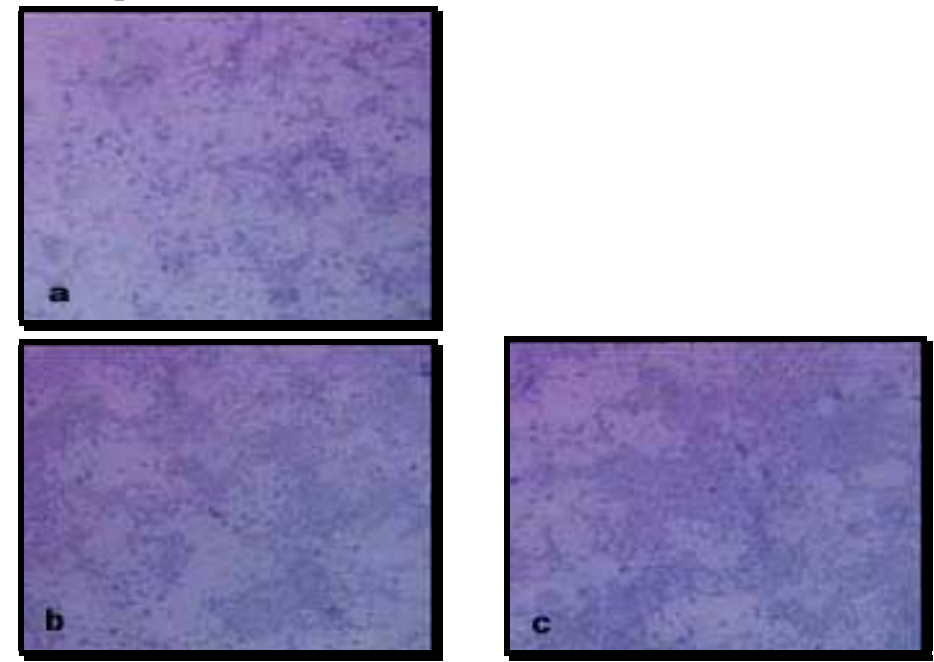

Fig.1: Distribution of EC adhering on Silcrothane with 10\% PDMS Fn-coated, after 3(a), 5(b), and 7 days (c). 
Discussion: We supposed that two mechanisms may explain the cells growth on the grafts. First, the Fn absorption is influenced by the surface free-energy or surface charge: hydrophilic surfaces accumulate less proteins then hydrophobic surfaces4. A second mechanism is the spatial arrangement of Fn, which is influenced by the substratum surface properties.

The EC growth rate increase may depend on Fn absorption and conformation arrangement on our polymers, so we are investigating the amount of Fn adsorption and the presence of cell binding sites by an ELISA.

This preliminary results are encouraging to develop an engineered vascular graft made by the new Silcrothane material and EC.

\author{
References \\ [1] Shi Q, Hong De Wu MH, Onuki Y, Ghali R and Hunter GC. Cardiovasc Surg 1997;25:736-42. \\ [2] Soldani G, Panol G, Goddard M, Sasken HF, Galletti PM. J Mater Sci: Mater Med 1992;3:106-113. \\ [3] Jaffe EA, Nacjman RL, Becker CG and Minick CR. J Clin Inv 1973;52:2745-56. \\ [4] Von Recum AF, Van Kooten TG. J Biomater Sci Polymer Edn 1995;2:181-98.
}

\title{
Characterisation of cardiac assist devices
}

\author{
S. Vandenberghe, P. Segers, D. De Wachter, P. Verdonck \\ Institute of Biomedical Technology, Ghent University, Belgium
}

Introduction: Patients suffering from chronic heart disease often evolve to a terminal stage where pharmacological treatment has no longer any effect. This is especially the case with patients suffering from cardiomyopathy, which is a disease of the heart muscle itself, not caused by valve failure or infarction [i]. Transplantation is the most common treatment, but since there is a major lack of donor organs, a lot of patients die while they are on the waiting list. A solution to this problem can be found in the use of Cardiac Assist Devices (CADs). CADs basically consist of a blood pump that is connected to the native heart and blood vessels of the patient in such a way that the heart is actively bypassed and the blood is forced through the body by the pump.

Such an active bypass gives the heart muscle the opportunity to rest since most of the work is performed by the pump. This unloading of the heart has already been reported to lead to recovery of the native heart function [ii]. This suggests that the device has potential to be used as a therapeutic instrument, rather than a temporarily bridge to transplantation.

An important issue is the selection of the best suited device and working mode for a specific clinical condition. Therefore, the aim of this study is to assess the hemodynamic characteristics of clinically used CADs to distinguish their most interesting working points.

Methods and results: A compact mock loop (fig. 1) was designed in which the systemic circulation is simulated by discrete components that each represent one characteristic property of the circulatory tree. The main components are a windkessel, mimicking the compliance (C) of large blood vessels, and a resistance $(\mathrm{R})$, mimicking the resistive effect of small arteries and capillaries. A small entrance resistance is placed before the windkessel, resulting in a 3-element windkessel afterload as defined by Westerhof et al.[iii]. A fourth element that can not be excluded is the inertia in the blood vessels, that 
is present in the mock loop mainly in the connection pieces between the components. Pressure and flow can be measured simultaneously at different locations.

The mock loop has been used to assess the characteristics of several rotary blood pumps and displacement pumps (Medos VAD, Novacor N100, Deltastream) with a water-glycerine mixture (60$40 \%$ ) and pig blood. For each test, different pump settings are combined with different settings of preload $(5-40 \mathrm{mmHg}), \mathrm{R}(0.3-6.8 \mathrm{mmHg} / \mathrm{ml} / \mathrm{s})$ and $\mathrm{C}(0.2-1.6 \mathrm{ml} / \mathrm{mmHg})$. This yields device specific pump function graphs (fig. 2) showing the relation between preload, afterload and pump flow. These graphs reveal how the different driving algorithms and working principles influence the hemodynamic properties.

An electrical analogous model is programmed in which the mock loop is reproduced by a modified 4-element windkessel model that has a series connection of an entrance resistance and an inertance. This model is matched to the mock loop measurements and to human data to evaluate the agreement between the discrete components and their physical counterparts.

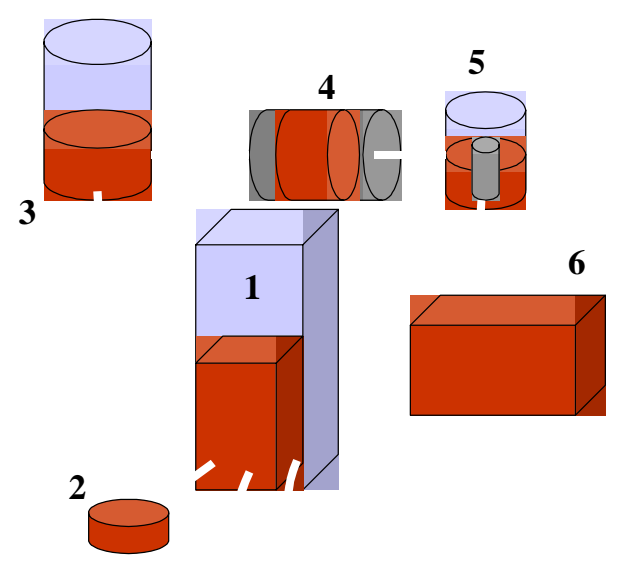

Fig. 1: Schematic representation of the mock loop: 1. preload reservoir 2. CAD 3. windkessel 4. resistance 5. afterload reservoir 6. buffer

The programmed model fits the mock loop data very well (mean RMSE $=1.22 \mathrm{mmHg}$, fig. 3), and the fitting of human data demonstrates that it is possible to link physical properties of the human body to the settings of the mock loop components.

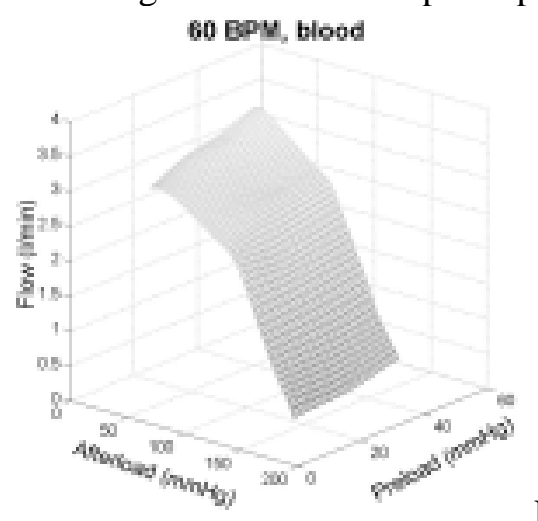

Fig.2: Flow in relation to preload and windkessel pressure (afterload) for the Medos VAD working at 60 Beats Per Minute (BPM) with pig blood 
Discussions and conclusions: A mock loop was developed that mimics the human arterial circulation. Physiologically relevant pressure and flow curves can be produced and the mock loop can be matched to a specific patient condition.

Initial tests have provided data on both rotary and displacement CADs and allow to discriminate between devices based on their maximum hydraulic power and preload and afterload sensitivity.

It was also demonstrated that a water-glycerine mixture is a good alternative for blood in hemodynamic CAD testing.

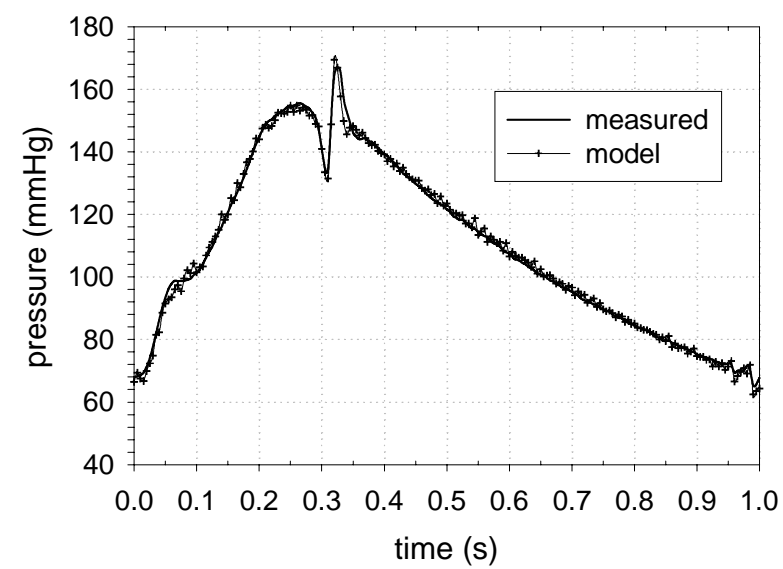

Fig. 2: Pressure-time curve in programmed model vs. mock loop measurements

\section{Acknowledgements}

This research is supported by a grant of the Flemish Institute for the Promotion of ScientificTechnological Research in Industry. (IWT-991171)

\section{References}

[1] Kühl U, Schultheiss HP. What is dilated cardiomyopathy. In: Hetzer R. HE, Loebe M., Mechanical Circulatory Support. Darmstadt: Springer; 1997:69-82.

[2] Müller J, Wallukat G, Weng YG, Dandel M, Spiegelsberger S, Loebe M, Hetzer R. Weaning from mechanical support after complete recovery in patients with idiopathic dilated cardiomyopathy. In: Hetzer R. HE, Loebe M., Mechanical Circulatory Support. Darmstadt: Springer; 1997:92-108.

[3] Westerhof N, Elzinga G, Sipkema P. An artificial arterial system for pumping hearts. J. Appl. Physiol. 1971;31:776781.

\section{Hemodynamic aspects of ventricle assist device}

I. Avrahami, S. Einav M. Rosenfeld ${ }^{\mathrm{a}}$ and K. Affeld ${ }^{\mathrm{b}}$

${ }^{a}$ Department of Biomedical Engineering, Tel Aviv University, Israel

${ }^{b}$ Humboldt University Berlin, Germany

Introduction: In many cases, a heart disease may be so advanced that there is no chance for patient to survive the wait for a donor heart. Therefore, the development of cardiac assist devices is of outmost importance to the survivability, and even to the rehabilitation of patients awaiting heart 
transplants. Temporary ventricle assist devices (VADs), which functionally replace the failing heart, are being used in increasing numbers.

Some of the most important factors that affect the longevity of the VADs are their hemodynamic properties. Interaction of blood with the moving components, stagnation regions or high shear stresses may promote thrombosis and hemolysis with detrimental effects on the operation of the VAD. The study of the flow field inside the VAD is, therefore, of importance both for optimising existing designs or developing new ones.

Methods: The presented research focuses on both experimental and numerical evaluation of the flow field inside an innovated VAD that is based on a new improved energy converter - the Berlin Left Heart Assist Device [1]. For this purpose, an experimental Continuous Digital Particle Imagining Velocimetry

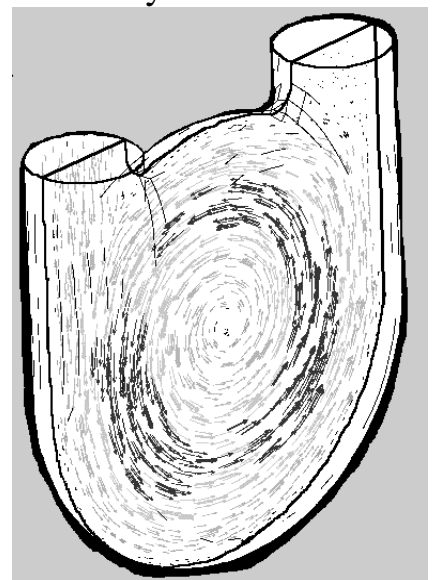

Fig. 1 Central cross-section of velocity vector plot at peak diastole in the numerical model of the VAD

(CDPIV) is combined with a computational fluid dynamics (CFD) analysis. These tools complement each other to result into a comprehensive description of the complex 3D, viscous and time-dependent flow field inside the artificial heart ventricle.

Numerical Analysis:A 3D numerical model was constructed to simulate the VAD pump and a timedepended CFD analysis with moving walls was performed to predict the flow behaviour in the VAD during the cardiac cycle. A commercial finite element package was used to solve the Navier-Stokes equations (FIDAP, Fluent Inc., Evanston) Fig. 1 shows a typical vector field inside the VAD.

Experimental Analysis: In the experimental analysis, an optically clear elastic model of the VAD was placed inside a 2D CDPIV system, see Fig. 2. The CDPIV system is capable of sampling 15 velocity vector fields per second based on image-pairs intervals lower than 0.5 millisecond, as described in reference [2].

Results: Continuous sequences of experimental images, followed by their calculated velocity and vorticity transient fields, are given as animated presentation of the distensible VAD. These results are used for validating the CFD simulations. Once validated, the CFD results provide a detailed 3D and 
time dependent description of the flow field, allowing the identification of stagnation or high shear stress regions.

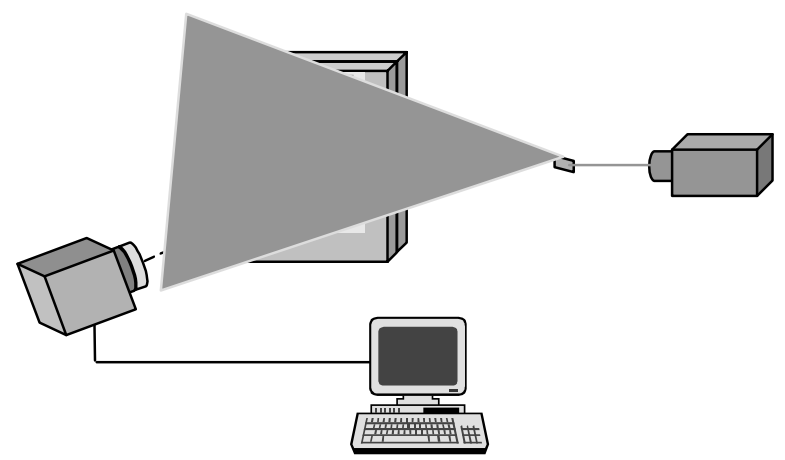

Fig 2: Experimental setup of the VAD for DPIV analysis

\section{Acknowledgements}

The authors wish to thank Mr. Sagi Raz, Dr. Uri Zaretsky and Dr. Ygael Grad for their assistance in the experimental analysis. The research is supported by a grant from G.I.F., the German-Israeli Foundation for scientific Research and Development.

\section{References}

[1] Affeld, K., A. Ziemann, and L. Goubergrits. Flow in Artificial Heart Valves and in Cardiac Assist Devices. in Ninth International Conference on Biomedical Engineering. 1997. Mandarin Hotel, Singapore: Biomedical Engineering Society.

[2] Y. Grad and S. Einav. "Spectral and instantaneous flow characteristics of vascular junctions using continuous DPIV". 9th International Symposium on Applications of Laser techniques to fluid Mechanics, Lisbon, Portugal, Jul 1998.

\section{Flow visualization. Experiments with a geometrically-optimized vortex blood pump}

C.E. Canal, J.R. Shanebrook, A.M. Anderson

Deparment of Mechanical Engineering, Union College, Schenectady, NY, USA

Introduction: One crucial objective in the design of an artificial heart device is the reduction of thromboembolic complications. Thus thrombus formation remains one of the largest challenges that face designers of these devices.

The Union College Vortex Blood Pump utilizes an airfoil-structured inlet valve, termed the hemofoil valve, to create a flow that mimics the vortex pattern found in the human heart. As presented by Lugt [1], Leonardo da Vinci was the first to discover the presence that the vortex has in nature and noted in particular the vortices generated by the leaflets of the human aortic valve. Furthermore, the vortical nature of blood flow through the left ventricle of the human heart contributes to its intrinsic self-cleansing mechanism.

In the Union College Pump the hemofoil valve promotes vortex formation upon fluid entry into the chamber during the diastolic phase. Recently the chamber of the pump was reduced in size in an attempt to increase the strength of the vortex and therefore its cleansing effectiveness. Experiments on 
velocities and flow patterns were conducted in order to measure the effects of this geometric optimization.

Methods: The in vitro testing apparatus consists of a Plexiglas pump model with a hemofoil inlet valve of $1.65 \mathrm{~mm}$ leading edge thickness, and $0.89 \mathrm{~mm}$ trailing edge thickness. A $29-\mathrm{mm}$ St. Jude bileaflet valve served as the outlet valve. Pulsatile test conditions were achieved with a mock circulatory system consisting of an atrial reservoir, steady flow reservoir, and flow capacitor. A motor-cam assembly provided for varying systolic/diastolic ratios. Three different cams were tested to find the most advantageous conditions for vortex formation.

Initial flow visualization testing was performed using water as the test fluid and neutrally buoyant Pliolite VT tracer particles. Tests were conducted at 60,90 and 120 BPM as well as with the three different cams (Sys/Dia of 1.00, 0.429 and 0.72). Results were recorded using a digital video camera and the MGI Video Wave III software package. Vortex formation was optimized at 60 BPM using cam \#1 with a systole/diastolic ratio of 1.00, as shown in Figure 1, and was also used for a more detailed analysis.

Through the use of correlation software, Particle Image Velocimetry (PIV) technology employs camera shots from successive frames of laser illuminated particles to map entire velocity fields. To enhance flow visualization and obtain quantitative as well as qualitative results a PIV system from TSI was used to make quantitative velocity measurements in the chamber. The PIV system consists of a dual mini Nd:YAG laser (30 mJ/pulse @532 nm), a computer controlled synchronizer, a PIVCAM 1030 digital CCD camera, which records $1 \mathrm{Kx} 1 \mathrm{~K}$ pixels at a rate of 30 frames per second, and image analysis software.

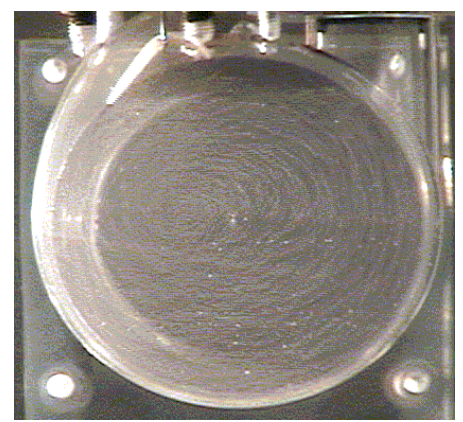

Fig. 1. Still frame obtained from Digital Camera shows the core of the shed vortex at about $2.0 \mathrm{~cm}$ below the edge of the hemofoil occluder.

Results: Decreasing the size of the chamber resulted in a significant increase in flow velocities. Previously, velocities within the chamber ranged from $0.22 \mathrm{~m} / \mathrm{s}$ to $1.20 \mathrm{~m} / \mathrm{s}$ [2] whereas with the new chamber, velocities can be found up to $3 \mathrm{~m} / \mathrm{s}$ averaging about $2 \mathrm{~m} / \mathrm{s}$. As can be seen from Figure 2, which shows instantaneous velocity vectors, the vortex forms at the inlet valve and continues to sweep the outside of the chamber, bringing with it the stronger velocities. PIV technology also allowed for an extensive study of the flow patterns and offered the advantage of pinpointing areas of high velocity.

Discussion: The correlation between vortex strength and size of the chamber is apparent and the decrease in the chamber size allowed for significantly higher velocities. However, there are limits dictated by a normal heart size as well as deleterious velocities resulting in hemolysis. The use of PIV techniques offers a new insight to the testing of the vortex and its flow pattern. As demonstrated, such 
technology as this can ultimately help model artificial heart devices more accurately after the human heart.

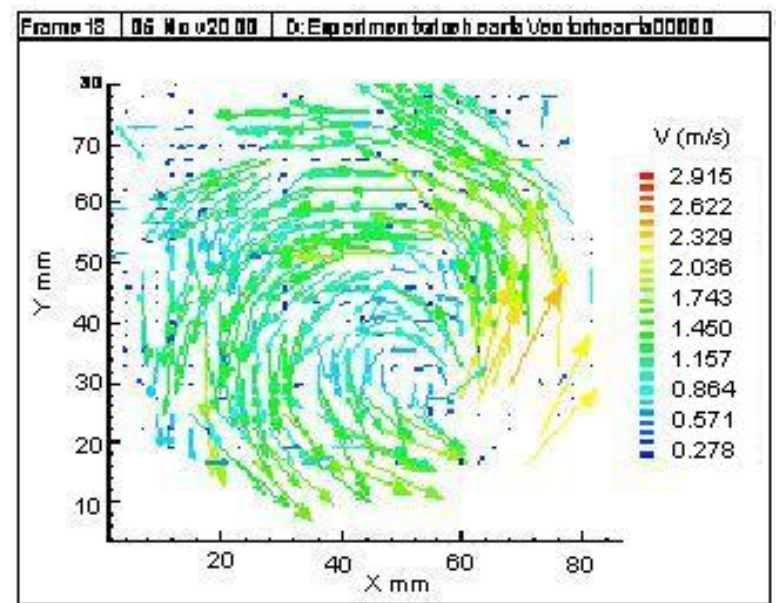

Fig.2. PIV results (an instantaneous image of the field using Tecplot) where vortex has made its way from valve in upper left to bottom of chamber

\section{References}

[1] Lugt H.J. (1983) Vortex flow in nature and technology. Wiley, NY, pp10-11

[2] Howarth C.B.(1998) Vortex Blood Pump. In: Akutsu T, Koyanagi H (eds) Heart Replacement - Artificial Heart 6: Springer-Verlag ,Tokyo, pp 74-77

\section{Session - Biomaterials Room 2, Thursday 3 May, 16.15 - 17.30}

\section{Determination of the quality of calcium phosphate bioceramics}

\section{A. Boyd, M. Akay and B.J. Meenan}

Northern Ireland Bio-Engineering Centre (NIBEC),School of Electrical and Mechanical Engineering, University of Ulster, Shore Road, Newtownabbey, Co. Antrim. BT37 OQB, Northern Ireland

Introduction: The intrinsic quality, i.e. the phase purity, percentage crystallinity, etc. of calcium phosphate $(\mathrm{CaP})$ materials is a key consideration for their effective utilisation in clinical applications. The effective bioactivity of a given system can be significantly influenced by the presence of secondary phases and other impurities. In particular, its ability to promote osteoconduction between bone and a CaP surface can be severely effected reduced.

Materials characterisation has obviously an important role to play in the provision of adequate quality assurance procedures which can be associated with the synthesis and subsequent processing of 
these materials. Various standards are currently being developed to provide for a definite bench mark for the properties of materials such as hydroxyapatite $(\mathrm{HA}, \mathrm{Ca} 10(\mathrm{PO} 4) 6(\mathrm{OH}) 2)$ and tricalcium phosphate (TCP, Ca3(PO4)2). Techniques such as X-ray diffraction (XRD) and Fourier transform infrared (FTIR) spectroscopy are commonly used to determine the composition and relative purity of $\mathrm{CaP}$ systems. However, in the "as-received" state, these data do not always provide a definitive assessment of these important materials properties due, in part, to poor signal-to-noise and peak broadening.

Thermal conditioning is often used to enhance the phase purity and crystallinity of $\mathrm{CaP}$ materials prior to their use. Hence, it is necessary to understand and control the effect that heating to elevated temperatures has on the properties of a given $\mathrm{CaP}$ system, particularly with regard to the onset of thermal decomposition. A systematic study of the effects of thermal processing on the chemical composition and structure could also be used to provide additional information about the nature and quality of the starting material.

Accordingly, in this study FTIR and XRD have been used in combination with X-Ray Fluorescence (XRF), Energy Dispersive X-ray analysis (EDX) and X-ray Photoelectron Spectroscopy (XPS) to determine the composition, crystallinity and phase purity of a range of $\mathrm{CaP}$ bioceramics before and after exposure to various thermal conditioning temperatures.

Methods: For the purposes of this study three commercially available and one laboratory synthesised $\mathrm{CaP}$ systems were used. The commercially available hydroxyapatite (HA) powders were obtained from Plasma Biotal Ltd. UK (CaP1), Merck KGaA, Germany (CaP2) and Sigma Aldrich, UK (CaP3). In addition, a laboratory synthesised HA type material (CaP4) was produced using a microwave irradiation assisted technique [2]. Samples of the powders were thermally conditioned in air for 2 hours at 400,800 and $1200{ }^{\circ} \mathrm{C}$ in a muffle furnace (Lenton, UK) with a heating ramp of $10^{\circ} \mathrm{C}$ per minute used to attain each soak temperature. The "as received" and thermally conditioned powders were analysed using FTIR (BioRad FTS 3000MX fitted with a Pike EasiDiff diffuse reflectance accessory), XRD (Siemens D5000), XRF (Spectro X-Lab 2000 ED-XRF), EDX (Oxford Instruments LINK ISIS fitted to a Hitachi S-3200N scanning electron microscope) and XPS (Kratos XSAM 800).

Results: From the XRD and FTIR results for the $\mathrm{CaP}$ powders, it is apparent that with the exception of $\mathrm{CaP} 1$, all the materials exhibit poor crystallinity in the as-received state. However, the poorly resolved diffraction data for $\mathrm{CP} 2$ and CP3 do show peaks similar to those expected for HA in accordance with the Joint Committee for Powder Diffraction Studies (JCPDS file 09-0432) database. However, peaks characteristic of $\mathrm{Ca}(\mathrm{OH}) 2$ are also observed in the XRD scan for CP4. This assignment is confirmed by the presence of a strong $\mathrm{O}-\mathrm{H}$ vibrational mode from at $3643 \mathrm{~cm}-1$ in the FTIR spectrum. Furthermore, the $\mathrm{Ca} / \mathrm{p}$ ratio in $\mathrm{CP} 1$ and $\mathrm{CP} 2$, as determined using EDX, are 1.70 ( 0.02 and 1.67 ( 0.02 , respectively. These values are very similar to that of stoichiometric HA (1.67). By comparison, the $\mathrm{Ca} / \mathrm{P}$ ratios of $\mathrm{CP} 3$ and $\mathrm{CP} 4$ are 1.40 ( 0.01 and 1.58 ( 0.02 , respectively.

As might be expected, heating the various materials to $400{ }^{\circ} \mathrm{C}$ has little or no effect on the various spectra. After heating to $800{ }^{\circ} \mathrm{C}, \mathrm{CP} 1$ shows little or no change in the spectral properties. However, $\mathrm{CaP} 2, \mathrm{CaP} 3$ and $\mathrm{CaP} 4$ all show FTIR spectra and XRD diffractograms with enhanced peak resolution. CP2 (Fig. 1) and CP4 (Fig.2) also show enhanced O-H vibrational modes at 3570 and $630 \mathrm{~cm}-1$. The XRD data for these materials suggests increased crystallinity for all of the materials on heating. However, in the case of the CP4, the XRD plot shows the presence of both HA and TCP. The FTIR spectrum for this material indicates the presence of additional PO43- bands, again evidence of the presence of TCP. The results for the $\mathrm{CP} 3$ material confirm that it is predominantly TCP. EDX analysis indicates no real changes in the $\mathrm{Ca} / \mathrm{P}$ ratios of the materials after thermal processing at $800{ }^{\circ} \mathrm{C}$. 
Heating the materials to $1200{ }^{\circ} \mathrm{C}$ enhances spectral resolution for all the systems studied. The FTIR results for CP3 suggest that significant dehydroxylation has occurred and confirms the presence of TCP along with HA as also indicated in the XRD data. Further evidence for the presence of both HA and TCP is observed in the XPS data. Similarly, the CP4 material contains both HA and TCP after heating to this temperature, however, FTIR and XPS analysis suggest the presence of additional $\mathrm{Ca}(\mathrm{OH}) 2$.

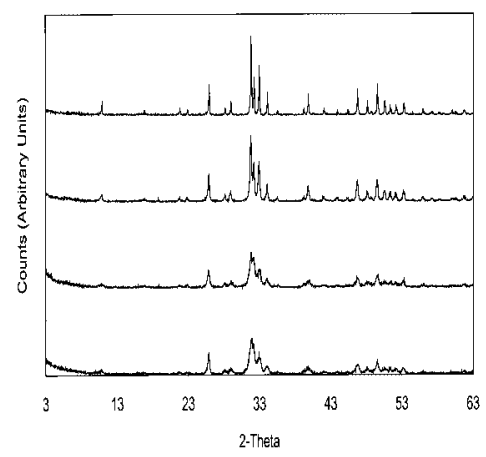

Fig. 1. XRD pattern for $\mathrm{CaP} 2$, (a) as- received, (b) $400{ }^{\circ} \mathrm{C}$, (c) $800{ }^{\circ} \mathrm{C}$ and (d) $1200{ }^{\circ} \mathrm{C}$.

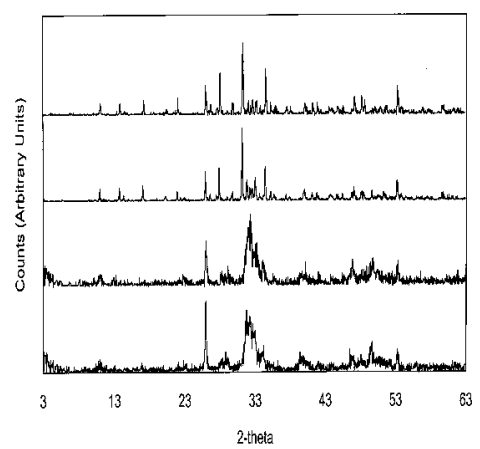

Fig. 2. XRD pattern for $\mathrm{CaP} 3$, (a) asreceived, (b) $400{ }^{\circ} \mathrm{C}$, (c) $800{ }^{\circ} \mathrm{C}$ and (d) $1200{ }^{\circ} \mathrm{C}$.

Discussion: This work highlights the importance of the full characterisation of Ca-P materials prior to their use. The commercially available and laboratory synthesised bioceramic systems studies here are not fully optimised for such purposes. With the exception of CP1, the as received materials are poorly hydroxylated forms of HA. The $\mathrm{CP} 4$ powder also contains significant levels of $\mathrm{Ca}(\mathrm{OH}) 2$.

Changes in the spectral data for the materials after heating clearly indicate that, whereas CP1 and $\mathrm{CP} 2$ are indeed HA, the other two specimens are not. The CP3 and CP4 samples contain TCP, the presence of which is only apparent after heating to at least $800{ }^{\circ} \mathrm{C}$. Hence, these results suggest that the $\mathrm{CP} 3$ and $\mathrm{CP} 4$ initially contain a non-stoichiometric and dehydroxylated $\mathrm{CaP}$, which decomposes to TCP on thermal processing. Furthermore, this decomposition begins at a temperature lower than expected for stoichiometric HA.

\section{References}

[1] C.J. Liao, F.H. Lin, K.S. Chen and J.S. Sun, Biomaterials 20: 1807-1813 (1999),

[2] B.Vaidhyanathan and J. Rao, Bul. Mat. Sci., 19: 1163-1165 (1996). 


\title{
Modification of medical implants by plasma immersion ion implantation
}

\author{
R. Sader ${ }^{\mathrm{a}}$, S. Mändl ${ }^{\mathrm{b}}$, D. Krause ${ }^{\mathrm{a}}$, H.F. Zeilhofer ${ }^{\mathrm{a}}$, G. Thorwarth ${ }^{\mathrm{b}}$ and B. Rauschenbach ${ }^{\mathrm{c}}$ \\ ${ }^{a}$ Department of Oral and Maxillofacial Surgery, University of Technology Munich, Germany \\ ${ }^{b}$ Institute for Physics, University of Augsburg, Germany \\ ${ }^{c}$ Institute for Surface Modification Leipzig and Institute for Experimental Physics II, University of \\ Leipzig, Germany
}

Introduction: It is well known that dense surface layers without pinholes and with excellent adhesion properties can be obtained using plasma immersion ion implantation (PIII). Another advantage of the method is the ability to treat the surface of complex three-dimensional objects simultaneously at a high dose rate, thus making it a fast and cost-effective process. When treating titanium or titanium based alloys like superelastic NiTi with oxygen PIII a rutile layer with its known good biocompatibility is formed over a broad temperature range starting near $200^{\circ} \mathrm{C}$. Using oxygenplasma immersion ion implantation (PIII) into titanium it is possible to form hard, dense and thick rutile layers with an excellent biocompatibility in a temperature region down to $200^{\circ} \mathrm{C}$. The thickness and crystallite size can be independently adjusted by changing the process temperature and the treatment time.

Methods: In this work the biocompatibility of PIII treated medical implants was tested. As a first step towards the application for osteosynthesis plates and dental implants used in jaw surgery the biocompatibility of rutile surface layers was tested using a rat model, which is well established for the testing of the biocompatibility of metal surfaces. Small rods with a diameter of $0.9 \mathrm{~mm}$ and a length of $10 \mathrm{~mm}$, prepared from titanium (grade 2) and two different NiTi alloys (NiTi SE 508, NiTi SM496), were

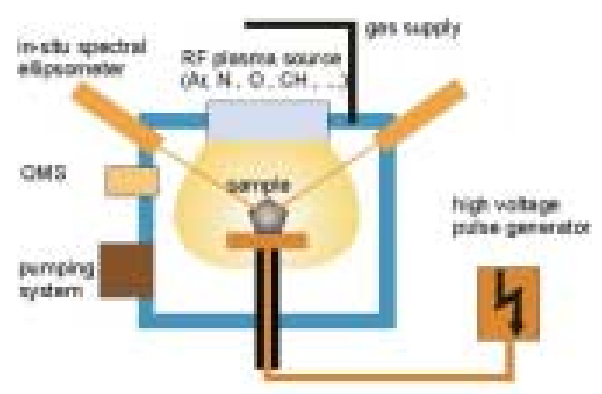

implanted with oxygen at temperatures between $200^{\circ}$ and $600^{\circ}$ with an incident dose of $5(1017 \mathrm{O}$ atoms $/ \mathrm{cm} 2$. Afterwards they were inserted into 96 rat femurs, together with untreated samples as a control group. An intravenous fluorochrome marking was performed to investigate the bone healing process. After 3 months the rats were sacrificed and pull-out test, histological and fluorescensemicroscopical evaluation were performed.

Results: The results of the biomechanical tests correlated to the histological results and proved, that plasma-immersions-ion-implantation leads to an increase of biocompatibility and osseointegration of titanium and titanium alloys. The following table shows the pull-out-forces (in $\mathrm{N}$ ) during extraction of the specimen from the rat femurs. 


\begin{tabular}{|l|c|c|c|c|}
\hline & Titanium & anodised Titanium & SM495 & SE508 \\
\hline Uncoated & 43.3 & Not measurable & 42.3 & 24.0 \\
\hline PIII-coated & 55.3 & Not measurable & 42.2 & 33.9 \\
\hline average & 49.3 & & 42.2 & 30.5 \\
\hline
\end{tabular}

Using oxygen PIII the formation of dense rutile layers with rather good adhesion on titanium was possible. The grain size and layer thickness could be independently controlled by varying the process time and temperature (see following figure).

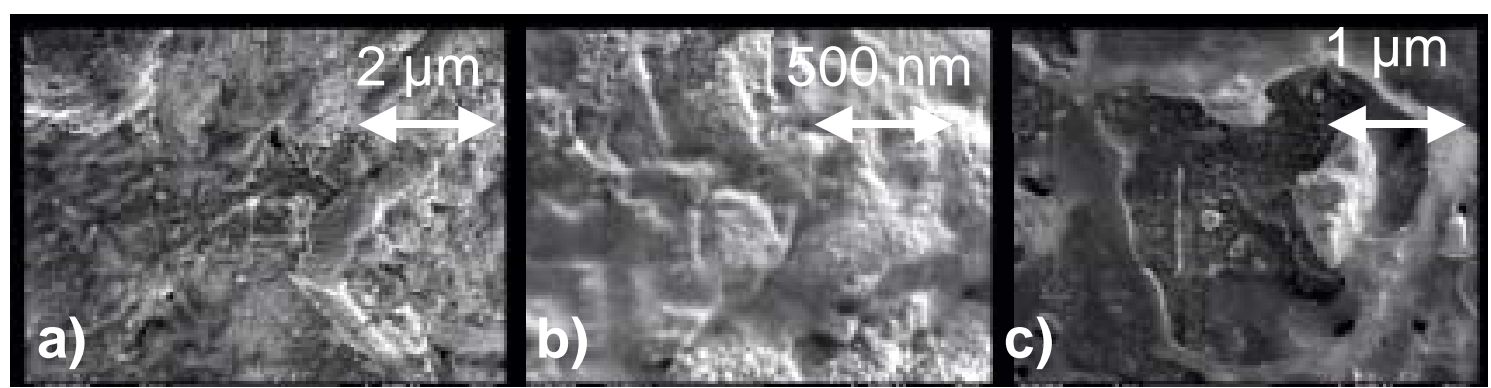

Fig.: Scanning electron micrographs of titanium samples after pull-out-test a) uncoated, b) $400{ }^{\circ} \mathrm{C}$ PIII, c) $600{ }^{\circ} \mathrm{C}$ PIII.

Comparing the osseointegration of different materials into rat femurs it was shown that still the main factor was the base material, however PIII treatment could improve the osseointegration. For further evaluation elastomechanical investigations of the bone-implant interface by scanning acoustic microscopy are presently in progress.

Discussion: The parallel between metabolite specific regional heterogeneity and respective normal standard deviations suggests that the relatively large signal variabilities found in normal population are indeed due to the regionally inhomogeneous metabolite distribution. While in the sequentially acquired spectra motional effects may dominate, slight VOI displacement by the operator is likely to influence reproducibility of separately acquired spectra.

A highly significant reduction of NAA concentrations found in the epileptogenic hippocampus allowed to lateralize the focus in accordance with invasive EEG monitoring in all TLE patients. The correlation with histopathology confirms the usefulness of in vivo 1H MRS as a non invasive clinical investigation tool.

\section{References}

[1] S. Mändl, G. Thorwarth, M. Schreck, B. Stritzker, B. Rauschenbach, Surf. Coat. Technol. 125, 84 (2000).

[2] R. Thull, K. Trautner, E.J. Karle, Biomed. Tech. 37 (1992) 162.

Address for correspondence:

Priv.-Doz.Dr.med.Dr.med.dent.Dr.med.habil. Robert Sader, Department of Oral and Maxillofacial Surgery, University of Technology Munich/Germany, Ismaninger Str. 22, D-81675 Munich/Germany. Tel.: 49-89-4140-2932; Fax: +49-89-4140-6233; Email: sader@mkg.med.tu-muenchen.de 


\section{The effects of sub optimal doses of mitomycin $\mathrm{C}$ on transitional cell carcinoma}

Godbole HC, Lynch TH, Anderson NH, Johnston SR, Keane PF, Hamilton PW, Arthur K, Williamson KE Department of Urology ,Belfast City Hospital \& Pathology Queen's University, Belfast, Northern Ireland

Introduction: To maximise the benefits of mitomycin $\mathrm{C}$ (MMC) and to minimise toxic and mutational side effects against transitional cell a better insight into its effects at a nuclear level is required. Previous studies demonstrated differential sensitivity to MMC induced apoptosis in bladder cancer cell lines with RT4 being most sensitive followed by T24/83 and HT1376 respectively. This study compared nuclear textural features, morphology and DNA flow cytometry in these 3 cell lines at day3 and day12 post incubation across a range of concentrations of MMC.

Materials and Methods: MMC was added to 50\% confluent cells at concentrations of 25, $2.5,0.25,0.025,0.0025,0.00025,0.000025 \& 0 \mathrm{ug} / \mathrm{ml}$ growth medium. Nuclear texture analysis was performed on Feulgen stained slides. Apoptosis was assessed on H \& E stained coverslips. DNA flow cytometry determined cell cycle characteristics. Statistical comparisons were made using SPSS software.

Result: Nuclear textural features were significantly different, apoptosis significantly higher and mitoses minimal at 25 and $2.5 \mathrm{ug} / \mathrm{ml}$ of MMC at day 3 when compared to the other concentrations in all three cell lines (Kruskal-Wallis p <0.001). By day 12 the cells exposed to 25 and $2.5 \mathrm{ug} / \mathrm{ml}$ were dead and $0.25 \mathrm{ug} / \mathrm{ml}$ showed textural features similar to $25 \mathrm{and} 2.5 \mathrm{ug} / \mathrm{ml}$ at day 3 . The nuclear textural patterns were very similar in RT4 and T24/83 cell lines, both being significantly different from HT1376.

DNA flow cytometry demonstrated significant cell cycle perturbation 25 and $2.5 \mathrm{ug} / \mathrm{ml}$ at day 3 in all three-cell lines.

Conclusion: The effect of MMC continued 12 days post instillation. There was a threshold dose below which cells were grossly unaffected by MMC. By day 12 the sensitive cell lines showed textural features similar to the resistant cell line. Nuclear texture feature differences may be associated with perturbations in the cell cycle. MMC in suboptimal doses induced sustained nuclear damage insufficient to destroy tumour cells while converting them into more resistant variants in vitro. This may have important implications in clinical practice.

The role of electrical properties and surface energy on the biocompatibility and haemocompatibility of si-dlc films

T. Okpalugo, A. Ogwu, P. Maguire and J. McLaughlin

NIBEC, School of Electrical and Mechanical Engineering,University of Ulster, Shore Road, BT37 $O Q B, N . I, U K$

Introduction: This paper presents the results of our preliminary investigations of a possible relationship suggested in the literature $[1,2,3]$, between surface energy, electrical properties and 
biocompatibility of Si-DLC. We have carried out extensive characterisation of chemically and physically modified Si-DLC films prepared by PECVD, using Raman and X-ray photoelectron spectroscopies, surface energy and electrical measurements. This is to be followed by biocompatibility and haemo-compatibility tests.

Materials and Methods: PECVD (RF: $13.56 \mathrm{MHz}$ ) DLC was deposited on silicon wafers and doped with silicon, using tetramethylsilane (TMS) vapour. The films were examined as obtained (both DLC and Si-DLC) and thermally annealed. AFM was used to obtain the films surface topography and the film thickness. The Raman and x-ray photoelectron (XPS) spectroscopies were carried out. A metalsemiconductor-metal (MSM) sandwich was prepared with Nordiko magnetron sputtering system and the bulk electrical property examined with a Keithley picoammeter. The contact potential difference (CPD)/work function (WF) studies were carried out with the Kelvin probe. The CAHN dynamic contact analyser (DCA) using the Wilhelmy plate technique and the KSV CAM 200 optical contact angle meter were used to measure contact angles and surface energy.

Results and Discussion: Raman spectroscopy shows an increase in ID/IG ratio with annealing temperature [4,5], but a decrease in ID/IG ratio with increasing amounts of silicon doping, a decrease in D- and G-linewidth with annealing temperature for DLC [4], and for lightly doped Si-DLC. There is a decrease in D-linewidth with annealing temperature for Si-DLC. The G-peak position increased and the D-peak positions decreased with increase annealing temperature for DLC, but for Si-DLC both the G-peak and the D-peak positions tend to increase with an increase in annealing temperature and decrease with increasing amounts of silicon. Raman Intensity Photoluminiscence was evident in SiDLC with annealing, and graphitization occurred at higher annealing temperatures $(>400 \mathrm{oC})$. Sidoping seems to lower the degree of graphitization.

The XPS analysis shows an increase in sp3/sp2 ratio with increasing silicon doping [6] but no obvious change in the binding energy except for the lowering of the B.E to $\sim 284 \mathrm{eV}$ where C-Si bonds are detected. Sp3/sp2 ratios of high silicon doped DLC seems to remain at about 0.5 on annealing.

The electrical resistivity measurements gave values of the order of ( $\sim 10 \mathrm{E}+11(. \mathrm{cm}$. Si-doping reduces the resistivity only slightly, less than one order of magnitude. The resistivity behaviour is nonOhmic and depends on the strength of the electric field. The resistivity simply decreases with increase in the strength of the electric field for DLC and with small amounts of silicon doping, but with higher amounts of silicon doping the resistivity seems to first increase with increasing strength of electric field up to a peak and then falls with further increase in the strength of the electric field. With the annealed films the resistivity drops in magnitude for both DLC and Si-DLC. At high silicon doping and higher annealing temperature the resistivity seems to vary less rapidly with increasing electric field strength. The resistivity of the annealed films falls in a simple fashion with increasing electric field strength. The WF and the CPD obtained from the Kelvin probe measurements falls with increased silicon doping. The WF decreases with an increase annealing temperature for DLC. There seems to be little change in the WF with increase annealing temperature of Si-DLC.

Si-DLC has a lower surface energy (higher contact angle) compared to DLC. The surface energy falls (contact angle rises) to a level where there seem to be no further fall with further increase in the TMS gas flow rate.

We are now commencing biocompatibility and haemocompatibility tests using various cell lines of both man and animal for in-vitro tests. This study is to find the optimal properties of these materials that yield optimal biocompatibility and haemocompatibility for implants and medical devices in any particular application [7]. 
Acknowledgement

T. O. acknowledges a University scholarship.

\title{
References
}

[1] S.D. Bruck, Nature, 243 (1973), 416

[2] C J Van Oss, Ann. Rev. Microbiol.,32 (1978), 19.

[3] M McLaughlin et al,Diamond and Rel. Mater. 5 (1996), 486

[4] Jin-Ting Jiu et al, J. Mater. Sci. 34 (1999) 5205

[5] Ogwu A et al, J.Phys.D: Appl. Phys. 32 (1999), 981

[6] Demichelis F et al, Mater. Sci. Eng. B 11 (1992), 313

[7] DF William, J. Biomed. Eng., 11 (1989) 185

\section{Control of surface morphology in calcium phosphate thin-films deposited by sputter deposition}

\author{
B.J. Meenan, A. Boyd and M. Akay \\ Northern Ireland Bio-Engineering Centre (NIBEC), School of Electrical and Mechanical Engineering, \\ University of Ulster,Shore Road, Newtownabbey, Co. Antrim, BT37 OQB, Northern Ireland
}

Introduction: Hydroxyapatite [HA, Ca10(PO4)6(OH)2], is one of a number of important bioactive calcium phosphate (Ca-P) materials which are used for the fabrication of medical and dental implant devices. However, their widespread clinical application is limited by poor mechanical performance in the bulk form. Many of these problems can be overcome, at least in part, by the deposition of $\mathrm{CaP}$ coatings onto biomaterials with appropriate mechanical properties. RF magnetron sputtering is one of the techniques that can be used for the production of such films and the technique gives a high degree of process control, thereby offering a significant advantage over other deposition methods, such as plasma spraying. The sputtering method produces thin film $(<1(\mathrm{~m})$ coatings that tend to follow the topography of the substrate during deposition. Hence, it is suggested that a number of key properties, particularly the resultant bioactivity, may be influenced and ultimately controlled by appropriate choice of sputtering conditions and deliberate manipulation of the substrate surface.

In this study, CaP thin films have been deposited from hydroxyapatite powder targets onto titanium substrate surfaces by RF magnetron sputtering. The substrates used were coupons of medical grade titanium metal, Ti-6Al-4V alloy and titanium surfaces prepared by sputtering from a metal target onto freshly cleaved mica and clean glass slides. Selected samples were subsequently annealed at 400 and 800(C in air for 2 hours.

All Ca-P coatings were examined by Atomic Force Microscopy (AFM) and further analysed by Xray Photoelectron Spectroscopy (XPS) and Energy Dispersive X-ray (EDX) analysis before and after HA deposition. Hence, the influence of substrate morphology on the chemical composition and surface structure of the films were determined before and after thermal conditioning.

Materials and methods: RF magnetron sputtering was performed in a high vacuum chamber equipped with a Torus 3M magnetron sputtering source (Kurt J. Lesker Ltd.) operating with a 13.56 $\mathrm{MHz}$ RF generator and an impedance driven matching network (Advanced Energy, UK). Sputter targets $(76 \mathrm{~mm}$ diameter and $5 \mathrm{~mm}$ thick) prepared from HA powder (Merck KGaA, Germany) were sputtered in argon at $10 \mathrm{mTorr}, 5 \mathrm{Sccm}$ and $150 \mathrm{~W}$ for 3 hours. The substrates $(1 \mathrm{~cm} \mathrm{x} 1 \mathrm{~cm})$ used were, as-received medical grade titanium foil, abraded (1200 grit SiC) Ti-6Al-4V alloy and titanium surfaces prepared by sputtering from a titanium metal target (99.999\%, Kurt J. Lesker Ltd., USA) onto freshly cleaved mica and rigorously clean soda-lime glass surfaces. The HA coatings deposited on titanium 
foil were subsequently annealed in air at $400^{\circ} \mathrm{C}$ and $800(\mathrm{C}$ for 2 hours. The various thin film coatings were evaluated by AFM (Digital Instruments Dimension 3000) operating in tapping mode; EDX (Oxford Instruments LINK ISIS system connected to a variable pressure Hitachi S-3200N SEM) and XPS (Scienta ESCA300 operating with Al K( X-rays).

Results: AFM images of the native titanium substrate surface show it to be comprised of irregular pitted features ( 10-20 (m across and several microns deep. A sputter deposited $\mathrm{CaP}$ thin film on this surface is also found to non-uniform and discontinuous, as indicated by the retention of the sharp edges associated with the flaws on the metal surface and the detection of spectral peaks assigned to titanium (Ti2p) in the corresponding XPS scan. The polished Ti-6Al-4V sample (Fig. 1(a)) has an irregular semi-crystalline surface topography with pronounced polishing scratches clearly visible. After HA coating of this material (Fig. 1(b)), pronounced columnar structures in the 200-800 nm range are observed. Deposition has therefore clearly occurred at surface defects, where the nucleation energy is lower. These HA layers have also been characterised after annealing, in air. After heating to $400{ }^{\circ} \mathrm{C}$, the surface becomes smoother and more continuous, retaining fewer of the features of the substrate. This is attributed to coalescence of the amorphous discontinuous calcium phosphate deposits. Further heating to $800{ }^{\circ} \mathrm{C}$ results in the formation of a $\mathrm{TiO} 2$ layer which grows through the $\mathrm{CaP}$ film.

The sputtered titanium layer on mica (Fig. 1(c)) shows crystallites ( $50 \mathrm{~nm}$ in size that form into distinct islands of clustered crystals ( $200 \mathrm{~nm}$ across. The tendency to form these crystal clusters is found to be more pronounced on the glass surface. The HA on the titanium coated mica surface (Fig. 1(d)), shows regular columnar microcrystallites of ( $100 \mathrm{~nm}$ in diameter. Higher magnification images indicate that these crystallites are inter-linked and lie in preferred, orientations.

XPS of the HA films on the alloy and titanium on mica/glass samples indicate that they are continuous with no evidence for signals from the substrate.

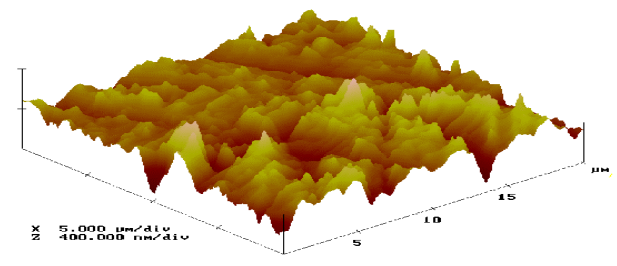

(A)

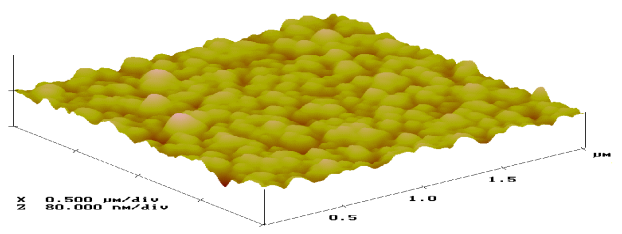

(C

Fig 1.AFM images of (a) polished Ti-6Al-4V, (c) HA on Ti-6Al-4V, (e) titanium sputtered onto mica,

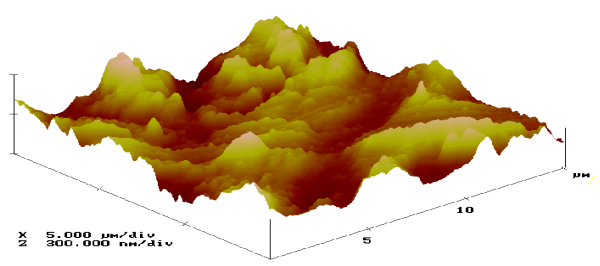

(B)

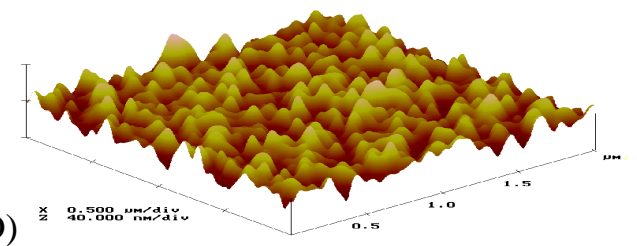

(f) HA on titanium-coated mica.

Discussion: AFM studies indicate that the morphology of HA thin films, deposited by RF magnetron sputtering on titanium is greatly influenced by the nature of the substrate surface. In the case of titanium metal, the film is deposited as a relatively flat, discontinuous layer which preferentially deposits at substrate fissures while for the Ti-6Al-4V columnar structures are formed around polishing scratches. For HA on titanium coated mica and glass, the film growth more closely mirrors the topography of the substrate. AFM and XPS results for HA coatings deposited on titanium 
and annealed at $400^{\circ} \mathrm{C}$ show an increased degree of ordering of the coating structure. Further heat treatment to $800^{\circ} \mathrm{C}$ causes a $\mathrm{TiO} 2$ layer to grow through the $\mathrm{HA}$ and dominate the surface chemistry. These results suggest that, by careful choice, or deliberate modification of substrate surface structure, it is possible to control the morphology of HA overlayers deposited by RF sputter deposition.

\title{
Session - Biosignals, Biosensors and Bioinformatics Room 2, Friday 4 May, 09.00 - 10.30
}

\section{Solving the direct problem of a nonlinear impedance equivalent circuit model}

\author{
J. Jossinet ${ }^{\mathrm{a}}$ and E.T. McAdams ${ }^{\mathrm{b}}$ \\ ${ }^{a}$ National Institute for Health and Medical Research, INSERM U556, Lyon, France \\ ${ }^{b}$ NIBEC, University of Ulster, Jordanstown, N. Ireland
}

Introduction: The impedances of electrode-electrolyte or electrode-tissue interfaces are, in general, non-linear functions of the applied signal frequency and magnitude. Such impedances can be modelled using Equivalent Circuit Models (ECM). Ideally, an ECM consists of the combination of discrete elements yielding the same frequency response as the original impedance. The nonlinear frequency response can be represented mathematically by a "constant phase angle" (CPA) element, $Z_{\mathrm{CPA}}(1)$. The impedance of such an element is given by equation 1 :

$$
Z_{C P A}=K(j \omega)^{-\beta}
$$

where $\omega$ is the angular frequency, $j$ the base of imaginary numbers, $K$ the magnitude of the $Z_{\mathrm{CPA}}$ element and $\beta$ the fractional power ( $\beta=1$ for 'pure' capacitance). The authors have shown that the behaviour of interface impedances can be described and predicted using an ECM consisting of $i$ / the parallel combination of a resistance, $R_{\mathrm{p}}$, and a constant phase angle element, $Z_{\mathrm{CPA}}$ and, in series with the above, ii/ a resistance, $R_{\mathrm{s}}$, representing the impedance of the media transversed by the injected current.

In the case of skin impedance, $Z_{\mathrm{CPA}}$ represents the dielectric coupling due to non-ideal capacitive properties of the stratum corneum and $R_{\mathrm{p}}$ represents the flow of the ionic current through the skin. In certain cases, the resistance $R_{\mathrm{p}}$ varies against the magnitude of the applied voltage (2). The present study describes the computation of the response of an ECM comprising a constant phase element in parallel with a nonlinear resistance.

Method: The presented method is based on the calculation of the response of the circuit to a current step. The computations were carried out in Borland Pascal DELPHI ${ }^{\circledR}$ using floating numbers of type "extended" supporting 18 significant decimal digits. The Laplace response of a $Z_{\mathrm{CPA}}$ element to a current step is given by equation 2 : 


$$
\mathrm{L}\{V(t)\}=Z_{C P A} * I(s)=Z_{C P A} * I_{0} \frac{1}{s^{1+\beta}}
$$

where $s$ is the Laplace variable. The inverse transform of this equation can be written under the form the time series of equation 3 (3):

$$
V(t)=\sum_{\mathrm{n}=0}^{\infty}(-1)^{\mathrm{n}} \frac{\tau^{n \beta}}{\tilde{A}(1+n \beta)}
$$

where $\tau$ is the normalised time ( $\tau=t\left(K / R_{P}\right)^{1 / \beta}$ ) corresponding to the used ECM topology. In practice, this series converges only for short times. For increasing times, another series expansion must used. Equation 4 was used in the present study:

$$
V(\tau)=\frac{1}{\pi} \sum_{n=1}^{\infty}(-1)^{n+1} \frac{\Gamma(n \beta) \sin \pi n \beta}{\tau^{n \beta}}
$$

The program automatically selected the appropriate equation using a look-up table based on the preliminary inspection of the outputs of equations 3 and 4 . The discontinuity at the transition was about $10^{-5}$. The $\Gamma$ function was computed using 300 terms for $\beta<0.5$ and 200 terms for $\beta>0.5$. This computation involved Euler's constant, $\gamma$. The approximate value of 0.577 , often reported in the literature was not appropriate for the present study. The iteration of Weierstrass equation for $\mathrm{x}=1$ (Equation 5) yielded a more accurate value $(\gamma=0.577215664914)$.

$$
g=\lim _{n \rightarrow \infty} \sum\left(1+\frac{1}{n}\right)-\ln (n)
$$

Results - Application: The accuracy of the computation of $\Gamma$ function was checked by comparison with the results of the analytical expressions available for value of $\mathrm{n} \beta=0.5,1,1.5$ etc. The error was found to be in the range $\left[-4.5 \times 10^{-12}\right.$ to $\left.+3.8 \times 10^{-12}\right]$ (mean $7.10^{-22}$ ). This software tool had several applications including, (Fig. 1), the study of the limit voltage of nonlinear charge transfer resistance (4) and the modelling of the step response of electrode-electrolyte interface (Fig. 2).

Conclusion: The computation of the response of a nonlinear equivalent circuit model of interface impedance provides a link between electrochemical (hence theoretical) and impedance (hence empirical) study of biomedical electrode systems. Further work include the calculation of the response to voltage steps and the calculation of nonlinear ECM elements from the experimental step responses (inverse problem).

\section{References}

[1] McAdams ET and Jossinet J: Bioelectrochem. and Bioenerg., 40:147-152.

[2] McAdams ET and Jossinet J: Med. \& Biol. Eng. \& Comput., 1994, 32, 126-13.

[3] de Boer RW and van Oosterom A: Med. \& Biol. Eng. \& Comput., 1978, 16, 1-10.

[4] Schwan HP: Ann.Biomed. Eng., 1992, 20, 269-288 


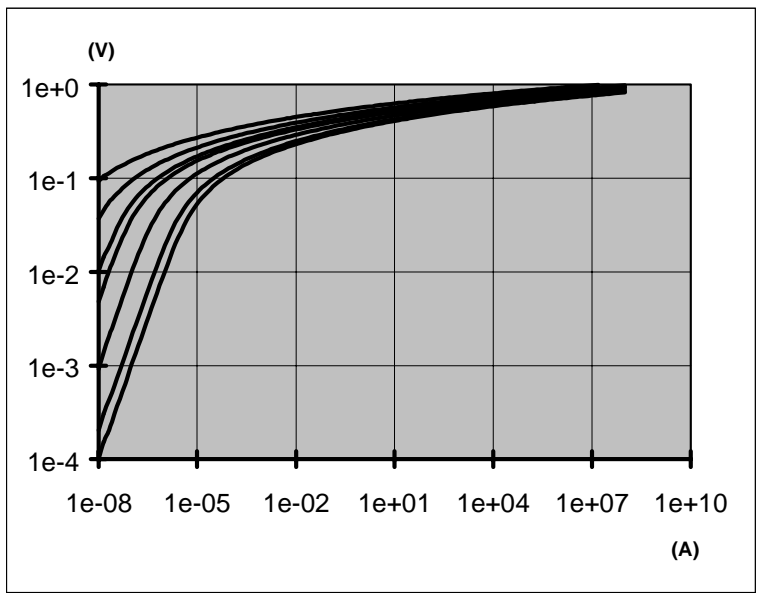

Figure 1 Limit voltage $(V)$ across a nonlinear resistance against applied current $(A)$ for $R p_{0}$ of $10 M \Omega$ (uppermost curve), $5 M \Omega, 1 M \Omega, 500 \mathrm{k} \Omega$, $100 \mathrm{k} \Omega, 20 \mathrm{k} \Omega$ and $10 \mathrm{k} \Omega$ (lower curves).

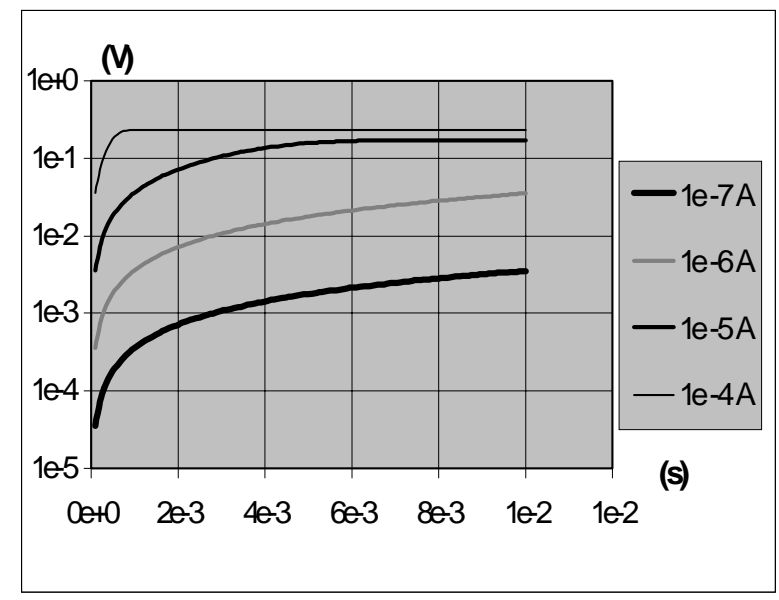

Figure 2 : Time response of a nonlinear ECM to a current step $\left(R p_{0}=1 M \Omega, K=22 \mathrm{k} \Omega . s^{-\beta}, \beta=0.75\right)$. The nonlinear behaviour results in signal "clipping" for a current of $100 \mu \mathrm{A}$.

\section{Nonlinear dynamics. From conformons to human brain}

W. Klonowski, E. Olejarczyk, R. Stepien 
Lab. of Biosignal Analysis Fundamentals, Inst. of Biocybernetics and Biomed. Eng., Polish Academy of Sciences, Warsaw, Poland

Introduction: Nonlinear Dynamics applies to systems that are not in equilibrium - from a single protein macromolecule with intrinsic physical instability (in scientific literature called KlonowskiKlonowska conformons [1-3]) and self-oscillating cross-linked (bio)polymeric materials [3-5], to nonlinear dose-effect relationships in Medicine (called hormesis [6]), and human brain [7].

Biomedical signals are generated by complex non-equilibrium systems. Human brain is a highly complex system that may show chaotic dynamics. That is why EEG-signal may have a fractal or multifractal temporal structure, while being inhomogeneous and non-stationary. That is why methods of Nonlinear Dynamics may be used to analyse changes in EEG-activity due to the applied chemo- , photo- or magneto-therapy. We search for characteristics that might be calculated relatively easily to facilitate assessing impact of therapy on patients and to help doctors in diagnostics.

Methods: Question arises is EEG-signal chaotic or stochastic. Our calculations using linear and nonlinear forecasting and method of surrogate data for nonlinearity tests show that hypothesis $\mathrm{H} 0$ that EEG-signal is generated by a linear stochastic process has to be rejected at least for some channels for any person (Fig. 1).

We think that the difference between "chaotic" and "stochastic" is quantitative rather than qualitative. Classical stochastic system would be one with an infinite number of degrees of freedom. If a system under consideration has many "irreducible" degrees of freedom it is still considered stochastic; if the number of degrees of freedom may be reduced to a few "really important" degrees of freedom, the system is considered to be deterministic-chaotic. In time domain, instead of speaking about degrees of freedom one should rather speak about sub-processes with different characteristic time scales.

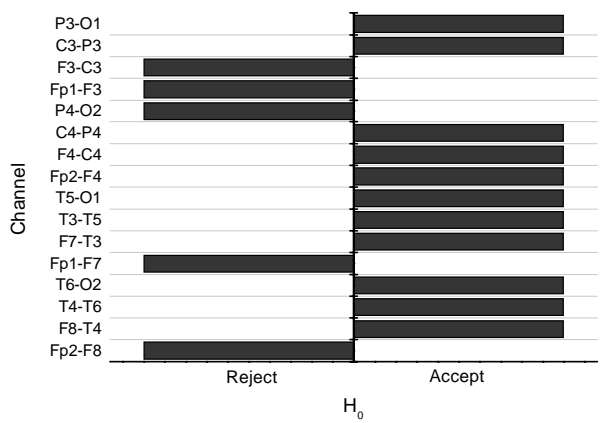

Fig. 1: Verification of hypothesis H0 ;"Rejected" means that the EEG-signal on a given channel is probably of nonlinear deterministic-chaotic nature.

But there exist methods introduced in Nonlinear Dynamics that may be applied to analysis of timeseries like EEG while taking no thoughts for signal "real nature". Between these methods are those that measure complexity of the signal in time domain (without embedding the data in a phase space) using e.g. fractal dimension of the EEG-signal curve on a plane, Df. Even trajectory of a Brownian particle (despite the fact that Brownian motion has always been used as a classical example of a stochastic process) may be characterised by its fractal dimension [8], similarly like a fractal sea-shore line. We choose Higuchi's algorithm to calculate fractal dimension. The calculations are simple and 
quick - raw EEG-data are transformed into Df(t) curve, one Df value for as few as 100 data points leading to 100 -fold compression of the original data. We have demonstrated that $\mathrm{Df}(\mathrm{t})$ helps to predict local FFT spectrum of the EEG-signal for a time interval around the time moment $t$.

Results: For EEG registered during sleep (together with other measured biosignals, like ECG, EOG, etc. called polysomnogram [9]) $\mathrm{Df}(\mathrm{t})$ calculated even on one channel enables categorisation of sleep segments into (at least three out of five) different steps (Fig. 2), i.e. it may be a help for a specialist in constructing so called hypnograms, which are important tools in diagnostics of sleep pathology and in assessment of chemotherapy with sleeping drugs (Fig. 2).

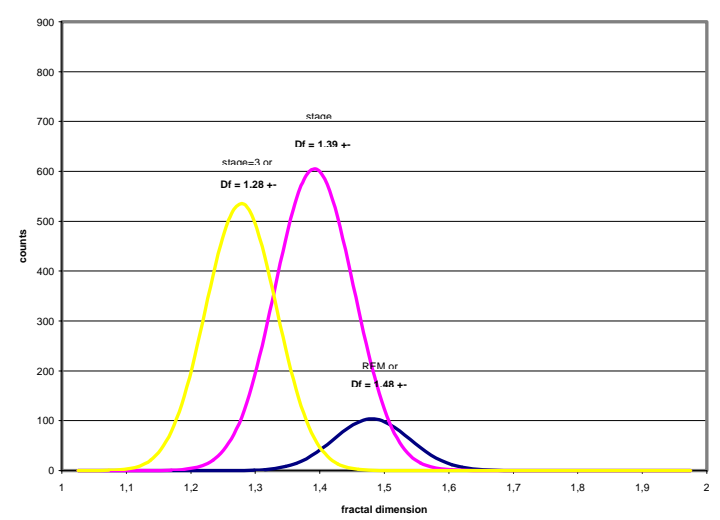

Fig. 2. Fractal dimension $(1<\mathrm{Df}<2$, abscissae) counted for different sleep stages

a. from a raw EEG-signal;

b. from the same signal with artefacts removed

Moreover, the method is prone to signal artefacts - the mean values of Df are practically the same, no matter if calculated from the raw signal (Fig. 2a) or from the signal with artefacts removed by a EEG-specialist (Fig. 2b). One "count" means a $4 \mathrm{sec}$. interval, because $4 \mathrm{sec}$. intervals are removed if contaminated with artefacts.

Its important to stress that even a specialist cannot categorise stage 3 from stage 4 , and stage 1 from REM based on EEG only; for that other biosignals from polysomnogram, such as EOG, are necessary.

Discussion: Because living systems work far from thermodynamic equilibrium (equilibrium means death) it is absolutely necessary to use Nonlinear Dynamics for analysis of processes in these systems and of the biosignals generated by these systems. Computer-assisted analysis of biosignals is more and more important in Medicine, for diagnosis and for therapy assessment.

\section{References}

[1] S.Ji, BioSystems, 54: 107-130 (2000).

[2] W.Klonowski, Computers and Chemistry, in press (2001).

[3] W.Klonowski, in: Self-Organization Of Complex Structures - From Individual toCollective Dynamics, F.Schweitzer, Ed., Gordon and Breach Publ. 235-239 (1997).

[4] W.Klonowski, Probabilistic Theory of Crosslinked Macromolecular Systems with Applications in Chemical Physics and Biophysics. Books on Demand AU00326, University Microfilms International, Ann Arbor, MI 48106 (1984). 
[5] W.Klonowski, in: Book of Abstracts, 4th European Conference on Engineering and Medicine (ESEM), Warsaw, P.Rabischong, J.Melin and M.Nalecz, Eds. 265 (1997)

[6] W.Klonowski, Biocyb.Biomed.Eng. 19: 41-55 (1999).

[7] W.Klonowski, J.Ciszewski, R.Stepien, W.Jernajczyk, K.Niedzielska, A.Karlinski, Book of Abstracts ESEM'99, Fifth Conference of the European Society for Engineering and Medicine, Barcelona, 163-164 (1999).

[8] W.Klonowski, R.Stepien, E.Olejarczyk, W.Jernajczyk, K.Niedzielska, A.Karlinski, Medical \& Biological Eng. \& Computing, 37, Suppl. 2: 436-437 (1999).

[9] M.Kaminski, K.J.Blinowska, W.Szelenberger, Electroenceph.Clin.Neurophys. 10: 216-227 (1997).

\section{Cellular separation by dielectrophoresis}

R. Casanella ${ }^{\mathrm{a}}$, O. Ruiz, M. Carmona ${ }^{\mathrm{a}}$, A. Juárez ${ }^{\mathrm{a}}$, J. Samitier ${ }^{\mathrm{a}}$, J. Bausells ${ }^{\mathrm{b}}$ and A. Errachid ${ }^{\mathrm{b}}$ ${ }^{a}$ Research Centre in Biolelectronics and Nanobioscience,Universitat de Barcelona, Spain

${ }^{b}$ Centre Nacional de Microelectrònica (CNM-CSIC),Bellaterra, Spain

Introduction: Dielectrophoresis (DEP) is a family of phenomena that imply the motion of electrically neutral matter under the influence of non-uniform electric fields. One member of this family is called common DEP (c-DEP) and is originated by electric fields inhomogeneous in amplitude. When an uncharged body is placed in a non-uniform electric field some electrical charges appear in its surface creating a dipole. The electric field aligns the dipole with regard to itself. As the field is non-uniform, one dipole end will be in a weaker region than the other. This originates a net force that causes the dipole to move towards (p-DEP) or away (n-DEP) from the region of highest field density [1].

DEP can be used in a big number of applications in the biotechnology and biomedicine fields such as particle characterisation, recollection and grouping of particles or particles handling. In this work we show the use of a dielectrophoretic system in particle separation.

Experimental setup: We have developed a microsystem that includes electrical, optical and fluidic interfaces using gold and platinum metals, silicon micromachining and photoresin patterning techniques (Fig. 1). The occurrence of DEP has already been successfully tested with polystyrene microbeads, yeast cells and bacterial cells [2]. And now we want to demonstrate the feasibility of cellular separation.

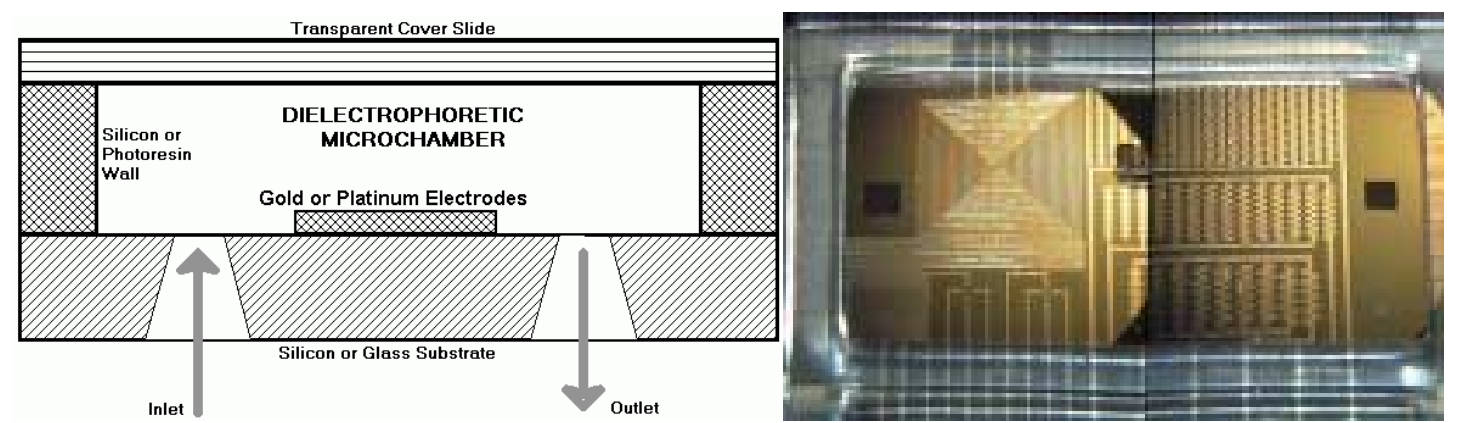

Fig. 1: Cross section sketch (left) and mosaic view (right) of the dielectrophoretic microsystem.

As a sample for the separation we have used a solution with saccharomyces yeast cells and escherichia coli bacteria (in concentrations of $107 \mathrm{ml}-1$ and 5.107 ml-1 respectively) in de-ionised water. 
An AC voltage of 5 volts in amplitude has been applied to the middle size interdigitated castellated electrodes where the squares' edges are $70 \mu \mathrm{m}$ long. The frequency of this voltage can be swept from 2 $\mathrm{MHz}$ to $20 \mathrm{MHz}$.

Results: Figure 2 shows two images where the separation of the different cellular components of the sample solution is evident. The image on the left has been taken when the AC frequency is around 2 MHz. In this condition both e-coli and saccharomyces undergo a p-DEP condition so that the high field densities of the electrodes edges attract all the cells in the solution. But the dielectric behaviour with the frequency of the samples is not the same. So there is a range of frequencies where one of the cellular types is under p-DEP and the other one is under n-DEP. This situation is reflected in the right side image of Figure 2. For an excitation frequency around $20 \mathrm{MHz}$ the e-coli cells exhibit a p-DEP behaviour but the saccharomyces are under n-DEP. That is why the yeast cells are moved away from the edges of the electrodes.

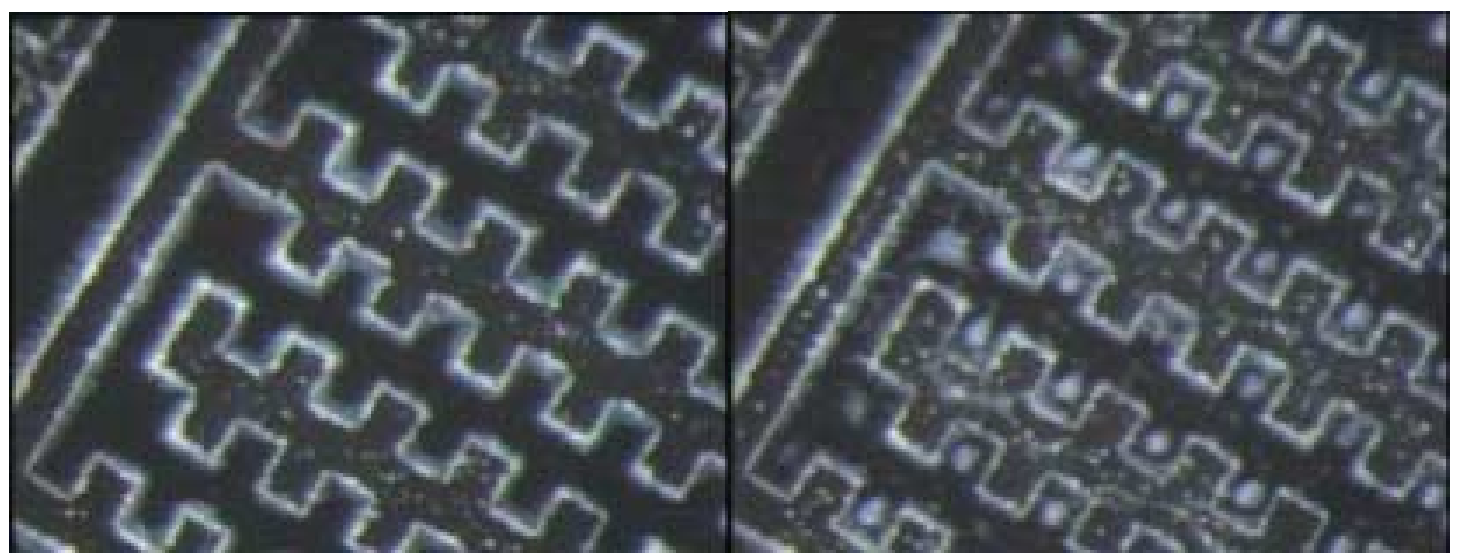

Fig. 2: Separation of e-coli and saccharomyces. The left image $(\mathrm{f}=2 \mathrm{MHz})$ shows both cellular types mixed. The right image $(\mathrm{f}=20 \mathrm{MHz})$ shows e-coli near the electrodes edges and saccharomyces away from the edges.

Discussion: The results obtained are in good agreement with the system modelling that lead us to the final design of the microsystem [1]. The effect of electric field on the fluid and its influence on the particles movement has yet to be considered in depth. The combination of modelling and experiments will give us insight into the nature of DEP microsystems and will guide us in the design of more efficient systems for various biological applications [3].

\section{References}

[1] N.G. Green and H. Morgan. J. Phys.D:Appl. Phys 30, L41-L44 (1997).

[2] F.H. Fernández-Morales, R. Casanella, O. Ruiz, M. Puig, A Juárez, J. Samitier, E. Abdelhamid and J. Bausells. BioMEMS \& Biomedical Nanotechnology World 2000

[3] J. Voldman, R.A. Braff, M. Toner, M.L. Gray amd M.A. Schmidt, Biophysical J. 80, 531-541 (2001)

\section{Potentiometric method for triglycerides determination}

D.G. Pijanowska and W. Torbicz 
Institute of Biocybernetics and Biomedical Engineering, Polish Academy of Sciences, ul. Trojdena 4, 02-109 Warsaw, Poland

Introduction: Triglycerides analysis is an indirect coronary risk factor. There are some methods for triglycerides or glycerol determination such as spectrophotometric as well as electrochemical. These methods are based on determination of glycerol as a product of lipolysis reaction [1-4].

This paper presents an indirect method of triglycerides determination based on $\mathrm{pH}$ changes detection, resulting from fatty acid production in hydrolysis reaction catalysed by lipase (1). The two methods of lipase immobilisation for the purpose of microsystem construction were compared.

Experimental: An enzymatic reaction was performed with use of lipase EC 3.1.1.3:

$$
\text { triglyceride }+3 \mathrm{H}_{2} \mathrm{O} \stackrel{\text { lipase }}{\longrightarrow} \text { glycerol }+3 \text { fatty acids }
$$

The measurements were taken in a flow system consisting of a $\mathrm{pH}$-sensor and microreactor. The lipase was immobilised on different supports and then packed in the microreactor. The enzyme was immobilised chemically by covalent coupling through glutaraldehyde and physically by adsorption onto nitrocellulose sheets and entrapment within alginate gel beads.

Materials: Lipase type II with the activity of $66 \mathrm{U} / \mathrm{mg}$, glautaraldehyde (25\% aqueous solution) and nitrocellulose sheets and aminopropyltriethoxysilane - APTS were obtained from Sigma. Triacetin, tributyrin and triolein purchased from Sigma were used as substrates for lipase. All other reagents applied here were of analytical grade and purchased from POCh, Gliwice, Poland.

Procedures of lipase immobilisation: Lipase was immobilised using two methods: 1) the chemical bonding to the surface of glass beads and 2) adsorption onto nitrocellulose sheets. The first immobilisation procedure consisted in enzyme coupling through glutaraldehyde to the glass beads treated with aminosilane. Prior to enzyme coupling, the glutaraldehyde activated glass beads were washed in vigorously stirred distilled water. Then, the glass beads were placed into the enzyme solution (10 mg of lipase per $1 \mathrm{ml}$ of distilled water) and left at room temperature for $2 \mathrm{~h}$.

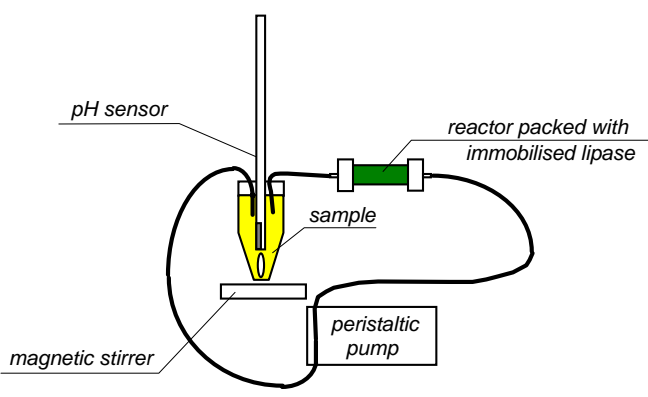

Fig. 1. Scheme of the measurement setup with the microreactor packed with immobilised lipase.

After completion of the Schiff's base formation, the non-attached molecules of the protein were removed by washing in stirred distilled water. In the second method, the enzyme was adsorbed onto the surface of nitrocellulose sheets. The solution containing lipase (50 mg of lipase per $2 \mathrm{ml}$ of $2 \mathrm{mM}$ trizma buffer solution) was dropped onto the NC sheets placed on the tissue paper and left for $1 \mathrm{~h}$. 
Afterwards, the NC sheets with immobilised lipase of surface ca. $4.8 \mathrm{~cm}^{2}$ were rinsed in trizma buffer solution, rolled up and packed into the microreactor.

Measurements: The measuring system consists of the $\mathrm{pH}$-sensor and microreactor operating in a flow system (Fig.1). The flow was driven by a peristaltic pump.

Results and Discussion: Two methods of lipase immobilisation were examined. Both methods of enzyme immobilisation based on lipase coupling onto glass beads and adsorption onto NC sheets are effective. However the first method of lipase immobilisation can be used for silicon based microreactors while the second one can be used for microreactors made by microengineering. Typical calibration curves for different substrates: triacetin, tributyrin and triolein are shown in Fig. 2. The sensitivity and linear ranges of the proposed $\mathrm{pH}$-metric method of triglicerides determination depend on the substrate (triglyceride type). The highest sensitivity was obtained for tributyrin $(0.478 \mathrm{pH} / \mathrm{mM}$ for concentration $<4 \mathrm{mM}$ ) while the widest linear range was obtained for triacetin (up to $30 \mathrm{mM}$ ).

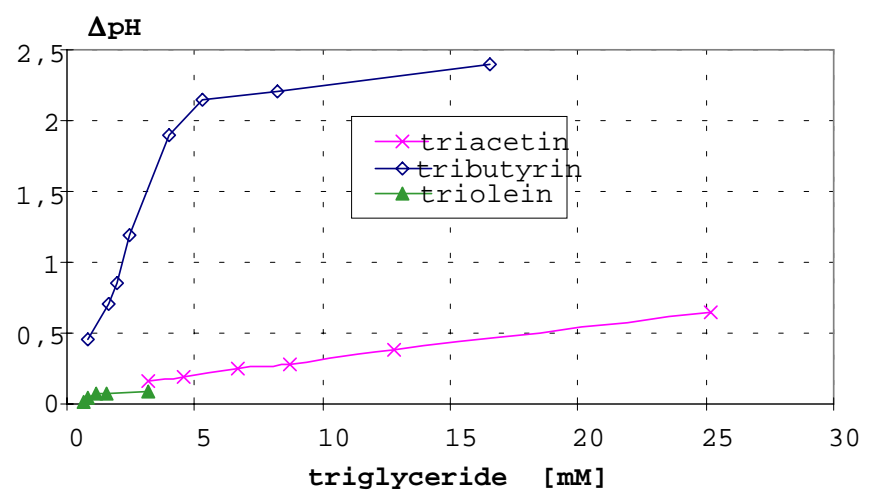

Fig. 2. Calibration curves for different triglycerides such as: triacetin, tributyrin and triolein.

Conclusions: The proposed method of the pH-detection of triglycerides can be used in clinical applications. The first method of lipase immobilisation based on coupling through glutaraldehyde can be used for silicon based microreactors fabrication i.e. for realisation of the final version of the microsystem consisting of microreactor made in silicon wafer with surface immobilised lipase.

[1] M. G. Gore, An automated enzymatic method for the determination of glycerol, Anal.Biochem., 75 (1976) 604-610.

[2] G. Bucolo, H. David, Quantitative determination of serum triglycerides by the use of enzymes, Clin.Chem., 19 (1973) 476

[3] S. H. Grossman, E. Mollo, G. Ertingshausen, Simplified, totally enzymatic method for determination of serum triglycerides with a centrifugal analyzer, Clin.Chem., 22 No.8 (1976) 1310-1313.

[4] G. Lehnus, L. Smith, Automated procedure for kinetic measurement of total triglycerides (as glycerol) in serum with the Gilford System 3500, Clin.Chem., 24 No.1 (1978) 27-31.

\section{Design of a urethral multi-electrode impedance probe}

J. Jossinet and E. Marry

National Institute for Health and Medical Research, INSERM U556, Lyon, France 
Introduction: Impedance measurement has long been used for the characterisation of body tissue using in diagnosis and monitoring applications, alone or in conjunction with other methods. In the treatment of localised prostate cancer, high intensity focused ultrasound causes the irreversible necrosis of the tissue. The evaluation of this lesion by ultrasonography is hindered by the low acoustic contrast between cancer and normal prostate tissue. The ability of impedance spectroscopy to discriminate cancer and normal tissue, well established in various body tissues $(1,2)$, yields a possible alternative evaluation tool. The objective is then to differentiate normal, pathological and treated tissue. The present study describe the implementation of a 16-electrode prototype impedance probe aimed at the in situ characterisation of the lesions produced in prostate by therapeutic high energy focused ultrasound.

Requirements: The final major features are the introduction of the probe into the urethra to make measurement at the prostate level. The electrode should then be applied against the internal wall of the urethra ensuring electrical contact but avoiding any tissue damage. In the final version, the probe must be compatible with sterilisation techniques. The measurement accuracy required for multi-channel impedance measurement is greatly dependent on electrode-tissue interface impedances and is highly dependent on electrode mismatch $(3,4)$. Therefore, precision mechanical machining of the electrode is required with small surface electrodes. The number of electrodes was taken equal to 16 since it did not create excessive implementation problems and enabled various injection strategies, including polar, adjacent and intermediate drives that yield different performance for image reconstruction (5).

Application - Results: The first step of the study was the construction of prototype at scale 5:1 (Fig. 1). The role of this prototype was to determine the fabrication and assembling processes of probe parts. This version consisted of a PVC cylinder $5 \mathrm{~cm}$ in outer diameter and $3 \mathrm{~cm}$ in inner diameter. At one end, 16 pieces of brass and their leads were fixed to the PVC tube and cast into epoxy resin. The resin and electrodes were then machined together to present a regular, smooth surface. The contact surface of each electrode was $2 \times 50 \mathrm{~mm} 2$. The capacity between adjacent electrodes was $0.85 \mathrm{pf}$. The contact impedance of each electrode was measured in a test bench constructed for this purpose (Fig. 2). This bench was also used to record the sensitivity distribution of the probe for future image reconstruction. The conductivity perturbation consisted in an insulating sphere positioned under the control of a microcomputer. The positioning accuracy was $0.1 \mathrm{~mm}$. The instrumentation consisted in a balanced current source and a differential amplifier, constructed based on author's previous developments of hardware systems and integrated active electrodes for electrical impedance tomography $(6,7)$. The output conductance of the current source was 0.85 S. The input impedance of the differential amplifier was $1 \mathrm{M} / / 1.5 \mathrm{pf}$. The contact impedance was about $1 \mathrm{k}$ per electrode.

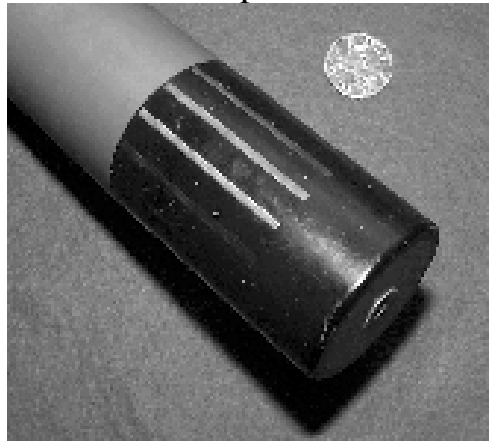

Fig.1: Prototype 16-electrode urethral probe. The diameter is $50 \mathrm{~mm}$. The electrodes are of brass. Contact surface is $2 \times 50 \mathrm{~mm} 2$ 


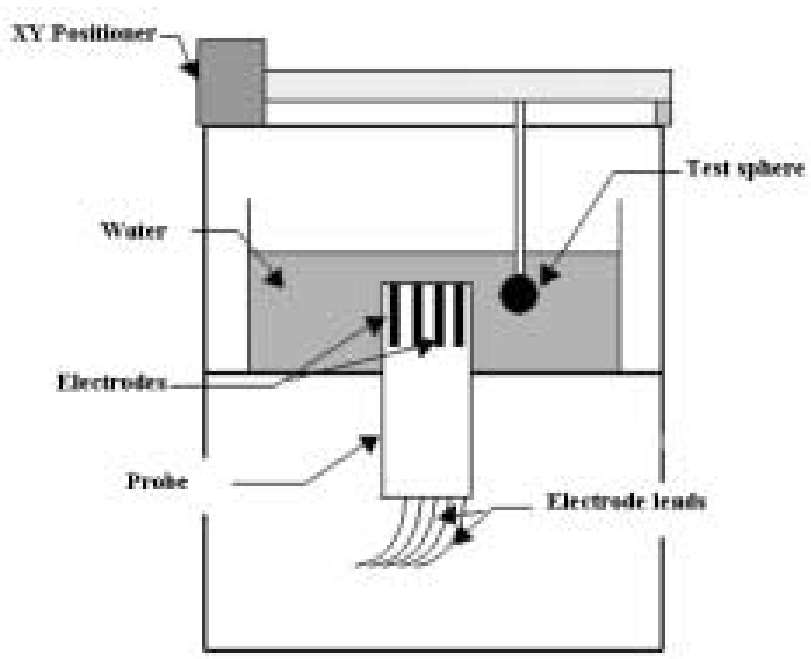

Fig.2: The test bench for performance evaluation of the probe and study of made the actual sensitivity distribution.

Conclusion: The prototype enabled to solve the problems related to the machining of the parts of the probe and to the assembling process. This approach facilitated the mechanical implementation, but resulted in increased electrode lead lengths that created excessive cross talk between adjacent channel, and therefore limited the test of the probe to low frequency. In the final size version, lead capacitances should be lower, but the electrode contact surface will be increased. The next step will be the evaluation of the influence of scale reduction on probe frequency response and instrumentation required performance. Further studies include sensitivity mapping, automated data acquisition and image reconstruction.

\title{
References
}

[1] K.R. Foster, H.P. Schwan HP, CRC Crit. Rev. in Biomed. Eng., 17:25-104 (1989).

[2] J. Jossinet, Proc. 4th Eur. ESEM Conf. Eng. \& Med., Warsaw, 196-197 (1997).

[3] E.T. McAdams, J. Jossinet, Proc. European Concerted Action on Impedance Tomography plenary workshop, Copenhagen, 14-19 (1990).

[4] E.T. McAdams, J. Jossinet, Automedica, 13:187-208 (1990).

[5] V. Molebny, J. Jossinet et al, Proc. ICEBI 10, Barcelona, 415-418 (1998).

[6] J. Jossinet, G. Tourtel, R. Jarry, Physiol. Meas., 15:A83-A90 (1994).

[7] J. Jossinet, G. Tourtel, R. Jarry, Proc. CAIT2 meeting "Hardware for multifrequency EIT", Toulouse, Innov. Techn. Biol. Med., 15(S1):40 46, (1994).

\section{Analysis and interpretation of complex HPLC/MS spectra for drug discovery}

\author{
H Zheng $^{\mathrm{a}}$, S McClean ${ }^{\mathrm{b}}$, P C Ojha ${ }^{\mathrm{a}}$, N D Black ${ }^{\mathrm{a}}, \mathrm{J} \mathrm{G} \mathrm{Hughes}^{\mathrm{a}}$ and C Shaw ${ }^{\mathrm{b}}$ \\ ${ }^{a}$ Faculty of Informatics, University of Ulster, Newtownabbey BT37 OQB, UK \\ ${ }^{b}$ School of Biomedical Sciences, University of Ulster, Coleraine BT52 ISA, UK
}

Introduction: Biomolecules of both plant and animal origin are potentially a rich source of novel drugs ranging from painkillers to cancer cures [1]. In the past 30 years, it has been discovered that the 
venom of a variety of frog and toad species contains a rich cocktail of potentially useful biomolecules. The research reported here contributes to the task of separating and identifying such molecules.

Proteins in the venom are separated and characterised in a high-performance liquid chromatography column (HPLC column) coupled to a mass spectrometer. The raw data comprises millions of 3-tuples, $(\mathrm{t}, \mathrm{m} / \mathrm{z}, \mathrm{a})$, where $\mathrm{t}$ is the retention time in the chromatograph, $\mathrm{m} / \mathrm{z}$ is the mass/charge ratio of the molecular ion and a is the relative abundance. Each biomolecule in the sample is associated with several hundred data tuples. The data analysis task is to identify the retention time and mass of real molecules from the morass of data. The mass-spectrum is exceedingly complex because of the following reasons (in order of decreasing importance): (i) the biological richness of the sample; (ii) poor signal/noise ratio from rapid scans; (iii) production of several charge states of a molecule on ionisation; (iv) fragmentation of molecules during ionisation and transport down the mass spectrometer; (v) the formation of positively charged ions by attachment of $\mathrm{Na}+$ or even $\mathrm{K}+$ ions instead of $\mathrm{H}+$, and (vi) the appearance of isotopic satellites corresponding to less abundant $\mathrm{C} 13$ and $\mathrm{O} 18$ rather than the most common isotopes $\mathrm{C} 12$ and O16 [2]. Some of these features are clearly visible in the spectrum in figure 1.

Analysis and interpretation of a complex mass spectrum with an interactive tool such as that provided by the manufacturer of the HPLC/MS instrument is exceedingly difficult and tedious, if not altogether impossible. Moreover, for drug discovery, one must identify common molecules in the spectra of several specimens from the same species. None of the available software addresses the second problem.

We will report on the development of algorithms for fully-automated analysis and interpretation of complex mass spectra and for comparison and identification of common molecules in several such spectrograms.

Method: In analysing the data we note that the retention time of a component is a random variable with a spread of about a minute and that the signal from a molecular ion of mass/charge ratio $\mathrm{m} / \mathrm{z}$ will be distributed randomly around this mean value with a spread of $0.2-0.3 \mathrm{amu}$. The noise comprises a background signal, which usually varies slowly over the entire run, and random spikes. The background signal is characterised by a mass of low-abundance data. Random spikes can be recognised by the fact that the signal is not sustained for 25-30 consecutive scans (i.e. the entire spread in the retention time of a component).

We have incorporated these elements in a fairly simple clustering algorithm which decides if a datapoint is the centre of a cluster and if not, assigns it to the nearest cluster centre. Further, it classifies each cluster centre as an ion or a noisy spike. Cluster centres which are classified as ions can then be examined further to see if they are different charge states of the same molecule etc. The clustering algorithm used here is simpler than the one reported previously [3] but is better suited to the analysis of mass spectra.

Results and Discussion: The output of the above clustering algorithm for raw HPLC/MS data from a known mixture of four peptides of masses 555.63, 571.67, 573.67 and $3465.0 \mathrm{amu}$ is shown in figure 1. The peaks at 556.11, 572.2 and 574.02 amu correspond to singly-charged states of the first three molecules. The peaks at 1732.58, 1155.77 and 867.04 amu arise from doubly-, triply- and quadruplycharged states of the fourth component. (The singly-charged state lies outside the range of the mass spectrometer.) The features at 578.22, 596.21, 1744.84 and 1163.34 arise from the addition of $\mathrm{Na}+$ rather than $\mathrm{H}+$. The remaining features may be satellite lines from the $\mathrm{C} 13$ isotope but are more likely to be noisy spikes that have not been removed by pre-processing. 
At the conference we will present further results from an extended algorithm incorporating (i) better removal of background noise and random spikes, (ii) improved determination of $\mathrm{m} / \mathrm{z}$, retention time and relative abundance by fitting the data in each cluster to a Gaussian distribution and (iii) postprocessing of the ion signal to identify the mass of the parent molecule.

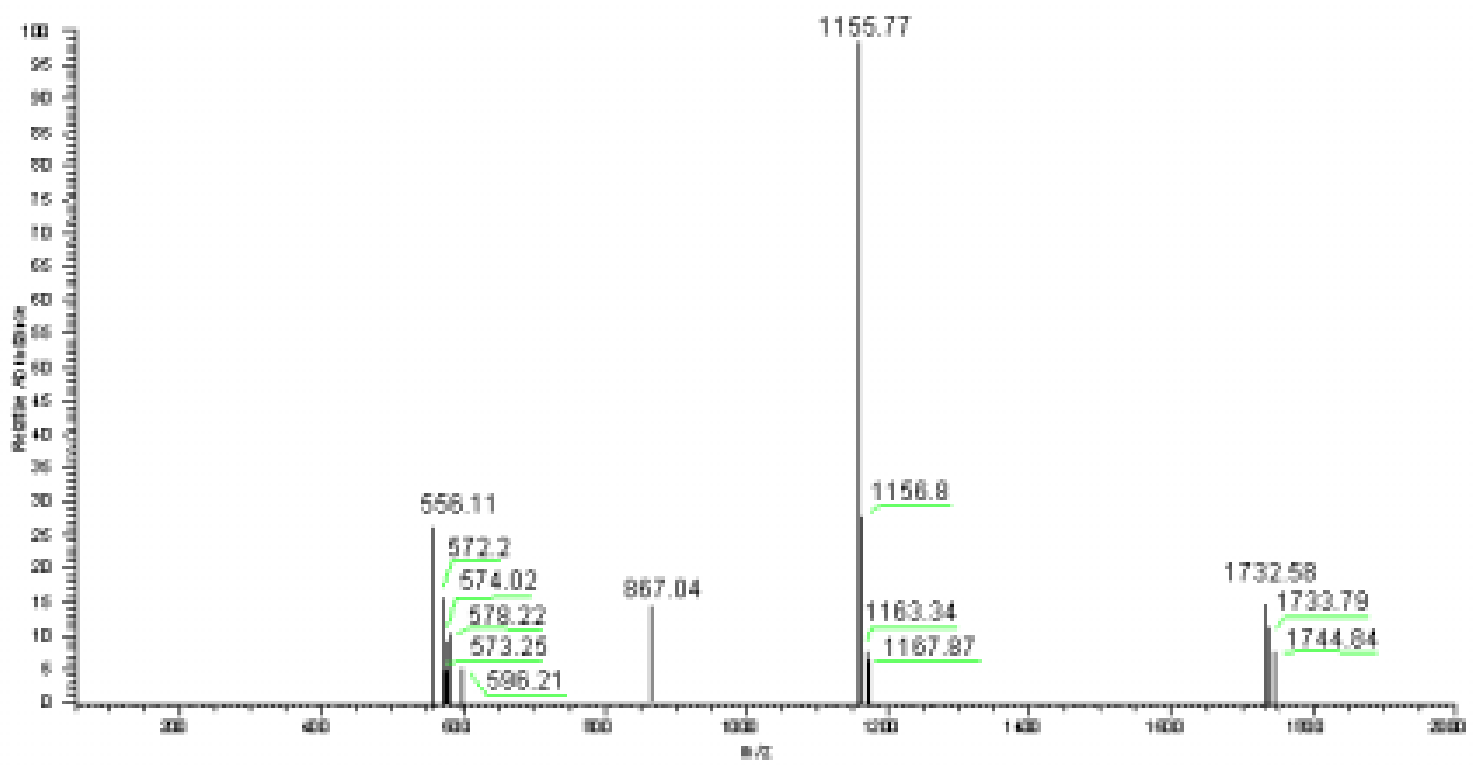

Fig 1: Results of analysis of a sample of known composition with four peptides.

\section{Acknowledgements}

We thank Robert Robinson for useful discussions and Philippe Lévi of ThermoQuest for his help in converting the data to text format.

\section{References}

[1] G L Patrick (1997) An Introduction to Medical Chemistry, Oxford University Press.

[2] W McLafferty and F Turecek (1993) Interpretation of Mass Spectra 4th Edition, University Science Books, California.

[3] H Zheng, S S Anand, J G Hughes and N D Black, Methods for Clustering Mass Spectrometry Data in Drug Development, ECAI 2000.

\section{Session - Rehabilitation \\ Room 2, Friday 4 May, 16.15 - 17.30}

From medical specifications to switching control implementation for a training-andrehabilitation machine for the lower limbs

L. Afilal, S. Moughamir, N. Manamanni and J. Zaytoon

LAM, Université de Reims Champagne-Ardenne, BP 1039, 51687 Reims Cedex 2, France

Tel: (+33).3.26.91.32.26 ; Fax: (+33).3.26.91.31.06 ; Email: janan.zaytoon@univ-reims.fr 
Background: Multi-Iso (Figure 1) is a computer-controlled machine for training and rehabilitation of the lower limbs. This machine, which is destined for medicine and sports, is the result of a joint project involving the Laboratoire d'Automatique et de Microélectronique (Reims-France) and the company Myosoft (Bellegarde-France). Multi-Iso is based on original concepts (Zaytoon, 1996) that provide significant improvements over existing machines, including precise rehabilitation adapted to user needs, and dedicated training possibilities to improve physical performance and autonomy (Moughamir, 1999).

A preliminary study has allowed us to establish the specifications of the sequences of control laws to be implemented for each training movement. The specific training sessions, defined by a physiotherapist with the help of a domain-specific man-machine interface, are translated into a corresponding switching sequence of force, position or speed control laws to be executed on a microcontroller. Training results are stored in a database to be subsequently processed. The control system comprises a PC-based supervisory module that handles the organisation and the co-ordination of the activities involved in the training sessions, and a micro-controller-based module implementing the switching control laws.

The architecture of Multi-Iso (Figure 2) comprises a software and control part, a mechanical part, and an electronic part. The functioning principle consists in applying a torque delivered by a brushless motor to one (or both) lower limb(s). This motoring action allows the user to attain a nominal force of 200 deca Newtons (daN) at the ends of the limbs and a speed of $360 \%$ sec under maximum load (Moughamir, 1999).

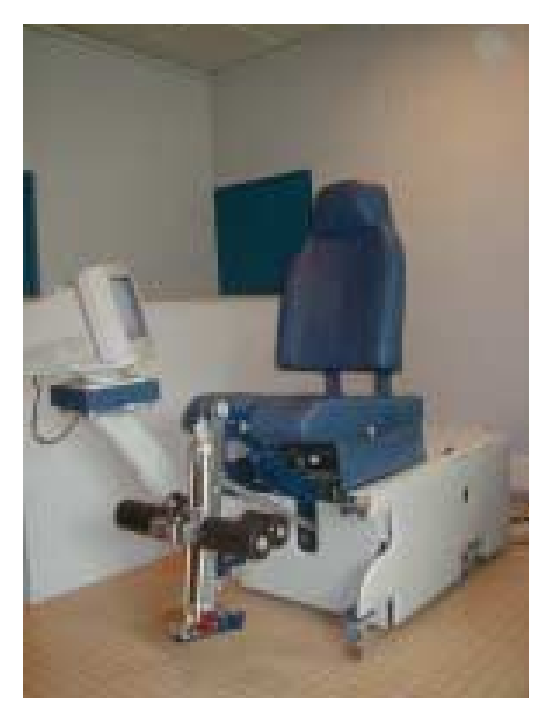

Figure 1. Multi-Iso

Organisation of the paper:The aim of this paper is to present the control system of Multi-Iso and to illustrate its performance through the use of a medical training protocol. The paper will be structured into three parts.

The functions of Multi-Iso will be reviewed in the first part to establish the requirements related to the control system. The original features of the machine in the medical and sports domain will be 
particularly emphasised. Multi-Iso supports concentric, eccentric or isometric movements, as well as seven training modes, four of which are original (steering, physiokinetic, stretching and assisted).

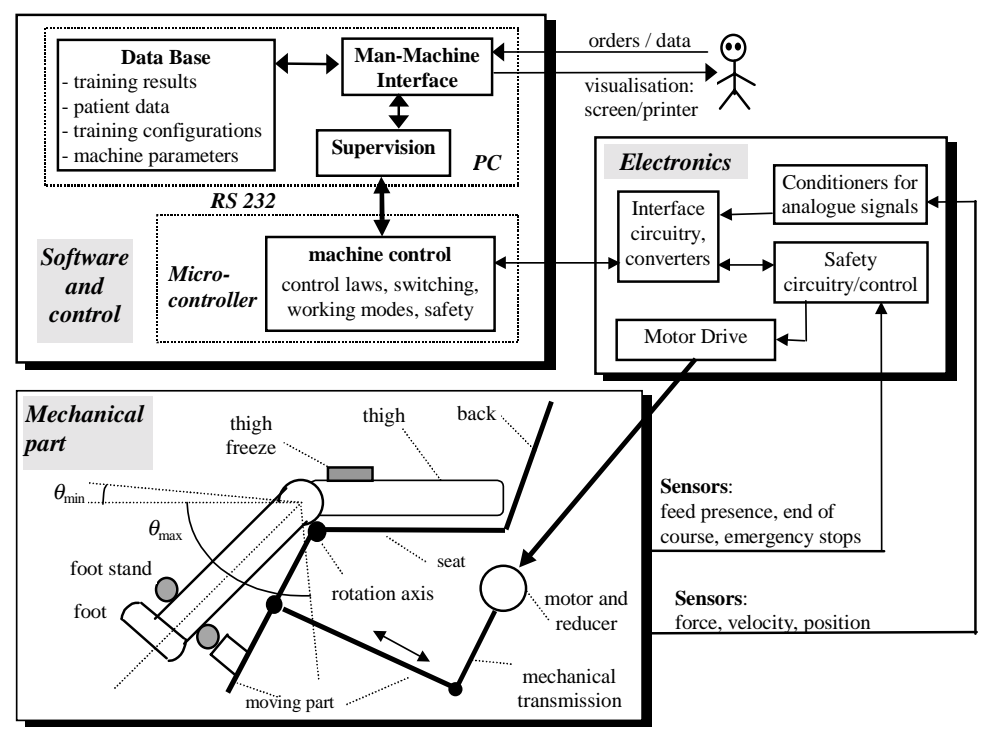

Figure 2. Architecture of Multi-Iso.

The second part of the paper describes the sequence of switching control laws required for each movement and training mode. The required position, velocity and force control laws are established using the dynamic model of a 1-DOF manipulator (Moughamir, 1998). A fuzzy velocity control law (Lee 1990) is used as an inner control loop for the position and force control laws. The force control law is based on original concepts with an objective to simulate the dynamics of a weight machine. In order to guarantee the continuity of the control variable when switching between the control laws, a practical solution based on the use of a numerical filter was developed to guarantee a smooth behaviour of the system, so as to ensure both the user comfort and the global stability of the control system. The dynamic behaviour of the machine subject to the switching control laws was validated in the first instance by simulation, and then throughout operational experimentation.

The third part presents some illustrative results for training sessions carried out by 10 sportsmen. These sessions followed a protocol devised by a physiotherapist to improve the muscular force of the quadriceps, through the execution of Isokinetic movement at a speed of $60 \%$. The physical exercises proposed here were carried out during a period of three weeks with four training sessions per week. They involved different types of movement.

These results illustrate the contribution of Multi-Iso in the sports domain, in terms of improving the force peak and the application range of the force of the sportsmen. They also demonstrate the adaptation capacity of the control system for each user.

\section{References}

[1] Albert, M. (1997). Entrânement musculaire et isocinétisme exentrique. Masson, Paris.

[2] Lee, C.C. (1990). Fuzzy logic in control systems: fuzzy logic controller - Part 1. IEEE Transactions on systems, man and cybernetics, 20(2), 404-418.

[3] Moughamir, S. (1999). Conception et développement d'une machine d'entrainement et de rééducation des membres inférieurs. PhD thesis, LAM-Univ. of Reims. 
[4] Moughamir, S., Zaytoon, J., \& Afilal, L. (1998). Modelling and analysis of an industrial hybrid system. Proc. 1998 IEEE International Conference on Systems, Man and Cybernetics. San Diego-USA, 851-856.

[5] Zaytoon, J., Richard, E. \& Angelloz, L. (1996). Spécification et conception d'un prototype asservi d'entraînement des membres inférieurs. Innovation et Technologie en Biologie et Médecine, 17(1), 57-70.

\title{
Combined magnetic and electric stimulation of peroneal nerve
}

\author{
E. Coyle ${ }^{\mathrm{a}}$, D.Curley ${ }^{\mathrm{a}}$, A de Paor ${ }^{\mathrm{b}}$, C Murray ${ }^{\mathrm{b}}$ and P Murray ${ }^{\mathrm{c}}$ \\ ${ }^{a}$ School of Control Systems and Electrical Engineering, Dublin Institute of Technology, Kevin St., \\ Dublin 2, Ireland \\ ${ }^{b}$ Department of Electronic and Electrical Engineering, University College Dublin, Belfield, Dublin 4, \\ Ireland \\ ${ }^{c}$ National Rehabilitation Hospital, D'Laoire, Dublin, Ireland
}

The technique of 'magnetic-electric stimulation' has been previously tried [Brickford et al 1987)]. Their results showed enhanced response using the two stimulators simultaneously, with conduction nerve signal amplitudes of two to four times greater than the response using the two stimulators separately.

Upon study of a nerve recruitment curve it is agreed that as stimulus intensity is increased more nerve fibres are recruited until all are firing, corresponding to the point of maximal response. If the stimulus intensity is increased above a threshold level (typically 80 volts) the response will be several times greater than at threshold value. Thus if a portion of currents from both stimulators combine together, it is clear that the response will be significantly greater than either in isolation.

In the course of the work presented in this paper an experimental design was set up combining use of a Dantec M2 magnetic stimulator and a Dantec Cantata EMG machine (to record response, together with a Medelec Ns6 electrical stimulator. For the experiment to be valid, the stimulating pulses should arrive either simultaneously or with a small fixed time delay between them. It has been shown that if a sub-threshold pulse has been applied, the threshold for stimulation by a subsequent second pulse will be reduced for a brief period, typically less than one millisecond [de Paor and Murray (1994)].

A method was designed to allow both stimulators be held in place without movement and without impeding upon each other. The peroneal nerve in the leg was chosen with the measurement electrodes placed on the tibialis anterior muscle. The leg was rested on a cushion to help avoid movement artefact interference.

Experiment Procedures and Results: Measurements were recorded as peak to peak readings of the recorded signals from the measurement electrodes. In order to ensure reliability the experiments were repeated on five different subjects, three male and two female with ages ranging between 20 and 30 years. Measurements were taken in suitable voltage increments using electrical stimulation between threshold level and a level that produced a maximal response or discomfort (a threshold response is the lowest noticeable response and a maximal is the maximum possible response). At each level of electrical stimulation measurements were taken using different levels of magnetic stimulation at 150volt increments of capacitor voltage from 300 to 1350 volts. Each measurement was repeated five times and the average result calculated. 
The combined stimulus response was greater (by more than a factor of four) to that achieved by using the stimulators separately. The time location of peaks was seen to be same for each stimulator. This demonstrates that the combined method is focal.

Attempts were made to model the interaction between the two stimuli, based on a linear singleinput, single-output, control system, with parameters gain and offset.. Factors were included in the model to account for variation in the level of magnetic stimulation. Results achieved showed that the model is a close representation of the measured system.

Following upon an analysis of data values obtained from the model, it was observed that

1. The gain of the system (i.e. effect of electrical stimuli) decreases in all cases for an increase in the level of magnetic stimulation

2. The offset in the system increases as the level of magnetic stimulation increases.

3. There was only one case (out of a total of fifty calculations) in which the gain of the system was negative, i.e. where an increase in electrical stimulus intensity caused a decrease in output from the system. This decrease was less than $2 \%$ of the maximum and occurred at maximal levels.

From these observations it is clear that the two types of stimuli do interact, and indeed constructively (producing an increased response).

\section{References}

[1] Barker A.T., 1987, "The magnetic way to brain stimulation", Elektor Electronics, June, pp 26-27.

[2] Hallett M., Cohen L.G., 1989, "Magnetism, a new method for stimulation for nerve and brain", J.A.M.A., Vol 262, pp 538-541.

[3] de Paor A.M., Murray C.D., 1997, "Simulation studies in rehabilitation engineering leading to simplification of the Hodgkin-Huxley neurone", Lekar a Technika, Vol 28, pp 51-60.

\section{Robotic orthosis improves treadmill training of paraplegic patients}

Gery Colombo,", Reinhard Schreier and Volker Dietz

ParaCare, Institute for Rehabilitation and Research, University Hospital Balgrist, Forchstrasse 340, $\mathrm{CH}-8008$ Zurich, Switzerland

Tel: ++41 138637 30; Fax: ++41 138637 31; E-mail: colombo@balgrist.unizh.ch

Corresponding author: Gery Colombo

Introduction: Manually assisted walking on a treadmill combined with body weight support has been used for more than ten years in the rehabilitation. It could be shown that this training improves walking capabilities 1, 2 in incomplete spinal cord injured patients (SCI).

To improve the possibilities of the locomotor training and to reduce workload of the therapists that were assisting the leg movements during training, the Lokomat, a driven gait orthosis, has been developed. It allows an automated moving of the legs of non-ambulatory patients during locomotor training on the treadmill.

First measurements in paraplegic patients seem to show that locomotor centers in the spinal cord can be activated and walking capability can be improved by the Lokomat .

Methods: Locomotor training was performed in incomplete and complete SCI with different levels of lesion with manual assistance or using the Lokomat. During both approaches the patients were 
standing on a moving treadmill, supported by a harness that was connected to a suspension system. The unloading of the patient's body weight was kept constant for the two training approaches.

The automated training was performed by the Lokomat3. The device was fixed to the patient by several straps fastened around the breast, waist and legs. The following parameters were variable, in order to allow an optimal fitting of the orthosis to the individual patient: width of the hip orthosis, length of thigh and shank, position as well as size of the leg braces. Two drives on each leg were moving the hip and knee joints of the orthosis and consequently the legs of the patient. The drives were controlled in such a way, that the legs became moved in a physiological gait pattern. Movement of the ankle joint was achieved by a passive foot lifter (see Colombo at al.3).

Two different approaches were used to prove effectiveness of the Lokomat:

1. EMG activity of leg muscles was measured during training. The rectified and averaged EMG data (Details see Dietz et al.4) was inspected visually and by calculating the variation ratio (VR) between the EMG signals obtained from the patients and a set of signals obtained from healthy subjects during walking.

2. Four functional gait tests were performed with incomplete SCI during the training period with the Lokomat to quantify the functional improvement.

Results:A visual and the statistical comparison between the modulation of the leg muscle EMG pattern in the patients with that of healthy subjects led to the following result: The muscles were more physiologically modulated during the automated training for both patients.

For the gait tests of the incomplete, chronic SCI, it could be seen, that a significant improvement in walking capability could be achieved during two month of training.

All patients that have been trained with both methods of leg assistance reported the automated training being more comfortable. The main reason for this being the more physiological gait pattern and the prolonged training.

Discussion: The success of locomotor training seems to be depending on the activation of neuronal centers within the spinal cord by an appropriate, cyclical afferent input4. The presented EMG data of the complete patient is of interest as in this case no voluntary intervention was possible but the recorded activity should be generated by the isolated spinal neuronal circuits only. It seems to demonstrate that with the Lokomat a similar or even better result can be achieved in comparison to conventional training. The data obtained from the gait test in chronic SCI confirms that a functional improvement could be achieved with a locomotor training by the Lokomat.

The conclusion is that a locomotor training with the Lokomat is effective. There seem to be several advantages of the automated locomotor training 1) Assistance of the leg movements is reproducible. This makes it possible to optimize the biomechanical gait pattern. 2) Training sessions can be prolonged and walking speed can be increased. 3) Workload of the therapists can be reduced.

\section{References}

[1] Barbeau H, Ladouceur M, Norman KE, Pepin A, Leroux A. Walking after spinal cord injury: evaluation, treatment, and functional recovery. Archives of Physical Medicine \& Rehabilitation 1999;80(2):225-35.

[2] Dietz V, Wirz M, Jensen L. Locomotion in patients with spinal cord injuries. Physical Therapy 1997;77(5):508-16.

[3] Colombo G, Joerg M, Schreier R, Dietz V. Treadmill training of paraplegic patients using a robotic orthosis. Journal of Rehabilitation Research and Development 2000; 37: 693-700.

[4] Dietz V, Colombo G, Jensen L, Baumgartner L. Locomotor capacity of spinal cord in paraplegic patients. Annals of Neurology 1995;37(5):574-82. 
Influence of walking speed, stride length and posture on ground reaction vertical force during human gait

Julio C. Politti ${ }^{\mathrm{a}}$, Xavier Savatier ${ }^{\mathrm{b}}$, Jean-Yves Ertaud ${ }^{\mathrm{b}}$

${ }^{a}$ Departamento de Bioingeniería (DBI), Facultad de Ciencias Exactas y Tecnología Universidad Nacional de Tucumán (UNT), Argentina

${ }^{b}$ Laboratoire d'Automatique Industrielle, Ecole Supérieure d'Ingénieurs en Génie Electrique, MontSaint-Aignan, France

Introduction: In human gait studies, inverse dynamic models have become a new way to analyse the relationship between the pathological disease of a subject and his walk [1]. Such models require data on the kinematics of the human body segments and the external forces [2], [3]. In the case of human gait, these only external forces on the subject are gravity and ground reaction forces (GRF). The kinematic data is usually measured by Video motion analysis systems and the GRF and momentum are generally given by force platforms.

By introducing the kinematic data and the external forces measurements in the equations of an inverse dynamic model, the net force and moment can be calculated in each joint. For inter and intraindividual comparison, these results must be normalised.

This is usually done by dividing the resultant force and moment by the total mass of the subject. Nevertheless this normalisation does not take into account the other gait parameters such walking speed, stride length and morphological data which may influence the results. To understand what the importance of these factors could be, we developed a theoretical 2D rigid model of the lower limbs and trunk (figure 1). By solving the equations taken from the dynamic laws, we obtained a relationship (equation 1) between the mean Ground Reaction vertical Force by unity of mass, and the four most significant gait parameters: walking speed $(\mathrm{v} 0 \mathrm{xcm})$, stride length $(\mathrm{p})$, relative position of gravitational center $(\mathrm{lcm})$ which takes into account the geometry of the human body and body posture ((). This equation was obtained by supposing the rigid body rotates around the extremity of the supporting foot $(\mathrm{O})$, strikes the ground and stops its movement.

$$
\frac{\bar{F}_{y}}{m}=\frac{. g . l \mathrm{~cm} \cdot \sin \left(\theta_{\max }+\theta_{0 \mathrm{~cm})}\right.}{p}+\frac{l \mathrm{~cm}}{p \cdot \Delta t} \sqrt{\left({ }^{20 x c m}\right)^{2}+2 . g \cdot l \mathrm{~cm}\left(\cos \theta 0 \mathrm{~cm}-\cos \left(\theta_{\max }+\theta 0 \mathrm{~cm}\right)\right)}
$$

The aim of this study was to investigate experimentally the influence of these parameters on the Ground Reaction vertical Force (GRVF) during the gait. In this presentation we report the results of experiments carried out with two subjects who walked with different speed, and stride lengths.

Methods:The experimental data was obtained in the Laboratory of Gait Analysis of the Centre Hospitalier Universitaire de Rouen (CHUR ) in France. Two male subjects without muscle-skeletal problems, different mass $(\mathrm{m} 1=60 \mathrm{~kg}, \mathrm{~m} 2=72 \mathrm{~kg})$ and height $(1.81 \mathrm{~m}, 1.72 \mathrm{~m})$ walked with their normal gait. Motion analysis was performed using a computer-aided video motion analysis system with five infrared cameras (VICON, Oxford Metrics Ltd., Oxford, England).The full body VICON Clinical Manager (VCM) protocol was utilized for the placement of 24 retro reflective body markers. 


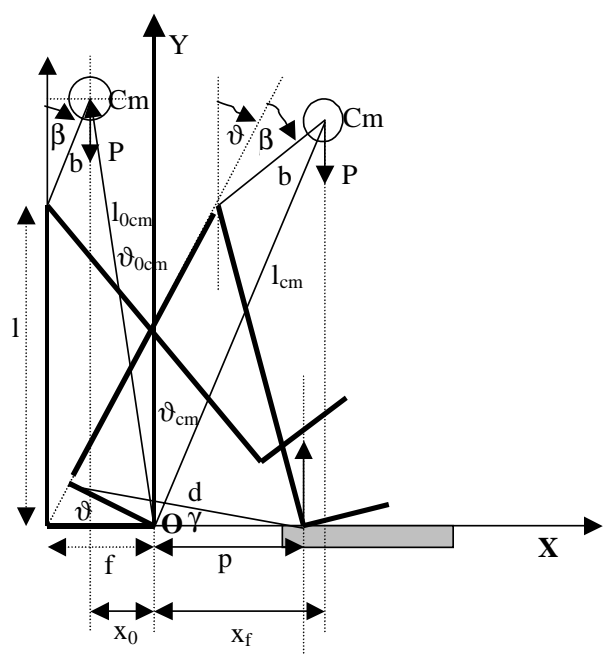

Fig.1: 2D rigid model of he lower limbs and trunk

The GRVF was measured with one force platform synchronized with the three dimensional video motion analysis system VICON. We fixed the stride length by placing markers on the ground and the walking speed by a digital metronome. Each subject walked with four different rates of march $(\mathrm{a}=65$, $b=75, c=85, d=95$ steps/min) and two different stride lengths: normal (SL1) and longer (SL2) corresponding to $(0.80,0.90 \mathrm{~m})$ for subject 1 and $(0.65,0.75)$ for subject 2 .

Results: The vertical forces measured by the force platform for one subject (subject 1) are presented in Figure (2) which shows for the two stride lengths, the amplitudes of the vertical forces for the different velocities. Figure (3) represents the vertical forces by unity of mass for the two subjects with the same set of parameters.

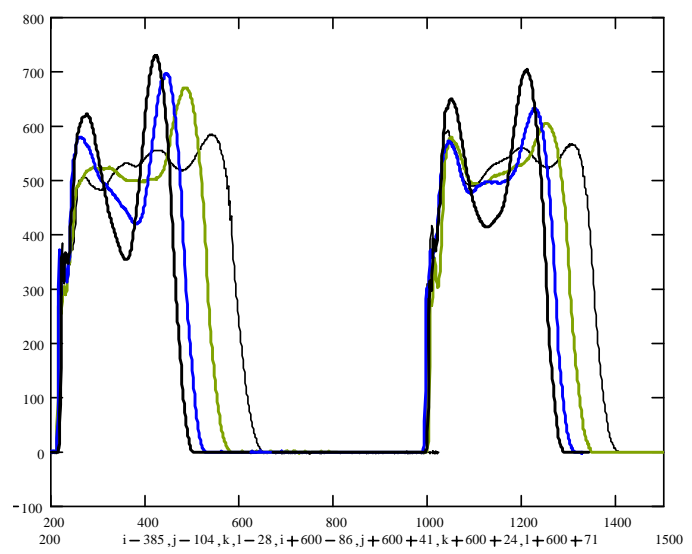

. Fig. 2 Amplitude of the GRVF for subject 1 with different speed and two stride lengths 


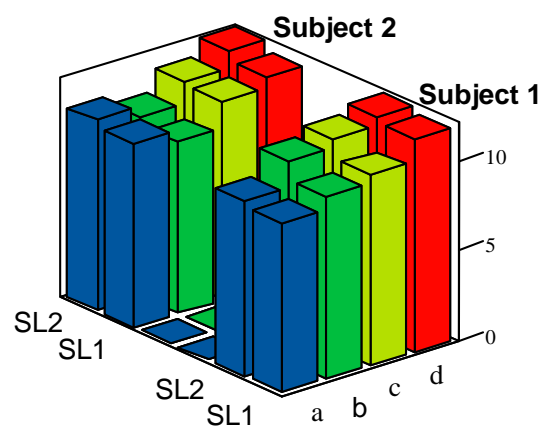

Fig.3: Amplitude of the GRVF by unity of for two subiects with different speed and stride

Discussion: The experimental results confirm our theoretical assumptions about the relationships between the vertical reaction force and the walking speed and stride length parameters.

The percentage of the change in the maximum value of the GRVF for a variation of the walking speed between $(0.97,1.42 \mathrm{~m} / \mathrm{s})$ and $(0.87,1.27 \mathrm{~m} / \mathrm{s})$ respectively for the subject 1 and subject 2 are $24 \%$ and $15 \%$. For the same rate of march the change of stride length produces a variation in the GRVF of $10 \%$ and $4 \%$ for the two subjects.

Our experiments demonstrate that a normalisation of the GRVF by the mass is not sufficient; the consideration of the walking speed, stride length and certainly the body posture is necessary in order to obtain this normalisation.

\section{References}

[1] C. L. Vaughan, Human Movement Science 15: 423-443 (1996).

[2] B. Bresler, J. P. Frankel, Trans.of ASME, 72: 27-36 (1950)

[3] D. A. Winter. ISBN 0-471-50908-6. Wiley \& Sons (1990)

\section{Responsibilities and involvement of players in the development of successful medical products}

C.J Snijders ${ }^{\mathrm{a}, \mathrm{b}}$, I.P.M. Griffioen-van Dijk ${ }^{\mathrm{c}}$, E. Vlaanderen ${ }^{\mathrm{a}}$, C.D Augustijn ${ }^{\mathrm{a}}$

${ }^{a}$ Department Physical Ergonomics, Faculty of Design, Construction and Production, Delft University of Technology, The Netherlands

${ }^{\mathrm{b}}$ Department of Biomedical Physics and Technology, Faculty of Medicine, Erasmus University

Rotterdam, The Netherlands

${ }^{\mathrm{c}}$ Indes Industrial Design \& Engineering, Hengelo, The Netherlands

Introduction: Von Hippel 2 described the interesting situation that medical doctors often are the inventor as well as the user of a medical product. The doctor experiences a need, contacts an engineer in his personal environment, tests a first prototype and transfers the result to industry. Finally he becomes the user of the commercial available product. This cycle can be repeated for the second or third generation of the product. This example of the innovation process illustrates that close interaction 
of developers and users is crucial to the successful development of new medical technologies. It also shows a form of "chain" in product innovation according to the following scheme:

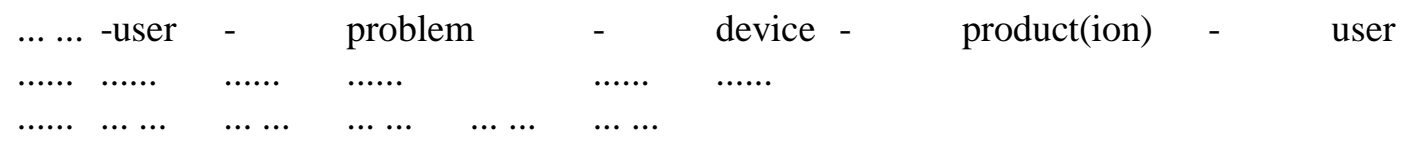

First question in this paper is who should be mentioned at the dots. Who has to be involved to get a successful product, who are the players in the complex field of medical technology development. In practice co-operation between different disciplines is not self-evident because problems exist on knowledge transfer between the links of the chain. Second question in this paper is if responsibilities can be identified for the initiation of such a "chain". The reason to discuss these questions is the introduction of a new concept for development in industry and the identification of gaps in responsibility in practice, with the need to implicate more players.

Table of players in development: In health care chain thinking dealt with bridging the gulf between mural and intramural care. This meant a stimulus for a new area, the home care technology. In this field the company Indes developed an easy and cost and time efficient method, the 'Tabling method' to include players from many disciplines in the process from product idea to marketing. This means users (patients, assistant workers, medical disciplines), producers, distributors, insurance companies, financiers, certification and legislation specialists, pressure groups and many others. All these players are involved in the team at the right time and with the right task, they deal on risks and business, influence the selection process and participate in communication and reports. Indes also developed the 'UCID method' (User Centered Industrial Design).This method involves the end users to enable designers to develop faster and more confidently a product that will be understood and appreciated by the

end users. The method also helps to establish more effective communication between marketing and design.

Responsibilities: In some medical situations we experienced that the innovation chain may be extended at its begin. Aim of this paper is to show that on the left side of the above diagram gaps exist with respect to responsibilities. We have chosen the following two examples from our research.

First example is that several reports in literature since 1968 mention lethal accidents caused by injection of Vincristine intrathecally instead of intravenously. Propositions exist, including procedural and instrumental measures, to solve that problem but at present no waterproof solution was found. We analysed the situation that pharmaceutic industry and pharmacy departments are not supposed to be responsible for such medication errors. But in our opinion it is also questionable if the medical profession can be hold fully responsible for situations related to poor technology with respect to safety. In any case it was not our responsibility to develop a safe and simple solution for administration of intrathecal chemotherapeutic agents by adaptation of the spinal needle. We found, however, a solution consisting of a new safety fitting for spinal needle and intrathecal syringe which should be implemented in all spinal instruments.

A second example is the development of an incubator for magnetic resonance investigation. The immature brain of preterm born infants is at increased risk of hypoxic-ischemic brain damage. For the diagnosis cerebral ultrasonography or magnetic resonance imaging are used. The importance of premature born neurological MR examination is, among others, to provide prognostic information about future quality of life. Through this prognosis the clinician is in a position to offer the parents 
better assistance to decide whether to continue treatment. Accessibility of the superior MR investigation, however, is impossible with the 24-hour baby incubator. We made an inquiry in several university hospitals to learn from earlier attempts to solve the problem and to compose a list of design criteria. Subsequently the design process started in which medical practitioners participated. We conclude that the incubator climate parameters air temperature, air humidity and oxygen concentration can be kept sufficiently stable in the MR environment and that for acceptance of MR examination ergonomics and aesthetics of the incubator are important issues as well. Although we perceived the urgent need for a MR incubator for which the necessary components and techniques are available and estimated costs seem to be reasonable, the question remains who is responsible for further initiatives.

The above examples emphasise the need of players who take responsibility for and who support the initiation of medical product innovation. So, to extend the above diagram at its begin.

\section{References}

[4] A.C. Gelijns. Innovation in clinical practice; the dynamics of medical technology development. Thesis, University Amsterdam (1991).

[5] E. von Hippel. The sources of innovation. New York, Oxford University Press (1988).

[6] C.D. Augustijn, W.S.C.J.M. van der Pol, C.J. Snijders, F. Koornneef. Prevention of accidental intrathecal administration of intravenous medication by adaptation of the Luer system for intrathecal use. Submitted.

[7] E. Vlaanderen, M.H. Lequin, C.J. Snijders. Incubator for MR investigation. Submitted.

\section{Session - Cardiovascular Biomechanics Room 2, Saturday 5 May, 09.00 - 10.30}

\section{The effects of arterial wall remodelling on the wall shear stress distributions in vascular bypass grafts}

O' Brien, T.P., Walsh, M., McGloughlin, T .

Biomedical Engineering Research Centre, Department of Mechanical and Aeronautical Engineering, University of Limerick,Limerick, Ireland

Local hemodynamic factors have been found to play a significant role in the development of atherosclerosis and intimal thickening at the distal end of arterial grafts [1,2]. The formation of disease at the distal end, in particular at the heel, toe and floor of the junction, reduces graft patency and may lead to occlusion of the junction. The investigation of junction hemodynamics serves to promote the understanding of how the blood flow patterns influence disease formation, thereby permitting optimisation of the graft-artery junction design to result in increased graft patency.

Previous Studies have investigated the effects of blood flow through idealised and realistic geometries on the wall shear stress (WSS) gradients and distributions. Results have shown that there is a significant correlation between areas of disease and abnormal WSS [3]. However, these studies do not account for the effects of arterial wall remodelling on the WSS gradients and distributions.

Arterial wall remodelling is part of the natural healing process and is an attempt to reverse the effects on the vessel wall of the altered flow patterns through the bypass junction [4]. This study addresses the issue of arterial wall remodelling by investigating the effects of geometrical alteration at the graft-artery junction on the WSS gradients and distributions around the anastomosis. 
Computational Fluid Dynamics (CFD) was used to simulate pulsatile, non-Newtonian flow through the graft-artery junction. The CFD packages FIDAP8.5 and Fluent 5 (Fluent Europe) were used to solve geometrical models created in ProEngineer (Parametric Technologies). The geometrical models were altered in ProEngineer where the artery diameter at the junction was gradually increased. Changes to incorporate constrictive remodelling were also made.

The results have found that there is a significant effect on the blood flow patterns and WSS due to the effect of arterial wall remodelling. As the artery diameter at the junction is increased, the peak WSS magnitudes decrease and the distributions change. Stagnation point movement is particularly significant when the artery undergoes a large degree of remodelling.

Arterial wall remodelling has a significant effect on the WSS around the anastomosis. The blood flow patterns through the anastomosis change, resulting in a shift in the stagnation point and altered WSS gradients and distributions. This changes the sites of disease formation. Since the degree of arterial wall remodelling depends on the time the bypass has been implanted, it is possible that the poor long term patency rates of vascular bypass grafts are caused in part by significant levels of arterial wall remodelling.

\title{
References:
}

[1] Hofer, M., Rappitsch, G., Perktold, K., Trubel, W., Schima, H., 1996. "Numerical study of wall mechanics and fluid dynamics in end-to-side anastomoses and correlation to intimal hyperplasia". Journal of Biomechanics, 29(10), 12971308.

[2] Fei, D. Y., Thomas, J. D., Rittgers, S. E., 1994. "The effect of angle and flow rate upon hemodynamics in distal vascular graft anastomoses: a numerical model". Journal of Biomechanical Engineering, 116: 331-336.

[3] Deplano, V., Souffi, M., 1999. "Experimental and numerical study of pulsatile flows through stenosis: wall shear stress analysis". Journal of Biomechanics, 32: 1081-1090.

[4] Rachev, A., Stergiopulos, N., Meister, J. J., "Theoretical study of dynamics of arterial wall remodelling in response to changes in blood pressure". Journal of Biomechanics, 29(5), 635-642.

\section{Mathematical model of the cardiovascular mechanics}

\author{
A. Martynenko, B. Kantor, M. Yabluchansky \\ National University, Kharkov, Ukraine
}

Introduction: Computer cardiobiomechanics is composed of mathematical modelling of cardiovascular system's functioning on the basis of biomechanics, its application in the form of programs for personal computers and the methodology of using derived results in medicine. Investigation in these fields gives a deeper understanding of the related laws in the functioning of healthy and pathological heart. Hence, it helps in diagnosis, optimizes treatment and prognosis of cardiovascular diseases. Therefore it is important during noninvasive patient's examination getting information about cardiovascular system, which for a today's are accessible only invasive or are inaccessible in vivo in general.

Model and Algorithm: The modelling structure in schematic looks as follows: hollow muscle bodies - chambers of the heart; the chambers of left and right heart are connected through valves; left atrium is connected through aortal valve with aorta; right atrium - with veins; aorta and veins after branching on the large vessels reaches peripheral capillaries. 
The system of equations, which needs to be decided in common at modelling of the cardiovascular system are [1,2]:model of soft biological tissues of cardiovascular; deformation of the heart chambers; deformation of the large vessels; pressure and blood flow in the vascular system.

Initial data: The aggregate of non-linear differential equations completely defines the cardiovascular mathematical model. Constants, that individualized a particular system, and the structure of equations, are required to certain of quantity of initial, boundary and coboundary conditions. The quantity and character of these conditions, determining algorithm of numerical realization of model, depends on a chosen technique of preparation of initial data, in this case noninvasive. All measurements are given by echocardiography, electrocardiography and sphygmomanometry.

Results: Combination of the computerized mathematical model and nonivasive patient examination permit quickly to define many biomechanical and haemodynamical functions and parameters of the cardiovascular system. There are pressure in left ventricle, atrium and aorta, volumes of left ventricle and atrium, blood speed through mitral and aortal valve, circumferance tension and active deformation on thickness of left ventricle's wall shown on the slide as characteristic functions.

General number of parameters received as a simulation result are 67. 26 parameters can be received invasively, 43 by various noninvasive methods, 11 - simulation only. Moreover unique opportunities of mathematical simulation give a number of new parameters inaccessible to other methods. For example, it is widely known hydraulic Efficiency of heart. We received expression for actomyisin Efficiency, giving real power assessment of heart works. Such data or distribution of active deformations in vivo can not be received by any other way.

Verification: The verification was executed on several directions. First of all, was established her physiological properties that is ability adequately to answer on preload, postload, chronoinotropy and contractility. Influences of the listed factors to parameters and functions of heart were considered.

Then were executed quantitative comparison of simulation results and direct invasive measurements. For example, in the [4] are shown the research of postload and contractility influence on the heart biomechanics. The results of numerical ( continuous line ) and experimental researches ( dash ) are given on the upper figure. The "pressure-volume" loops are shown in initial state and after achievement of a given level of postload. On the lower figure are indicated similar results, but with previously changed of contractility. The loops 2 are received by iteration from initial state 1 at step-by-step change of postload on $0.4 \mathrm{ml}$ increment.
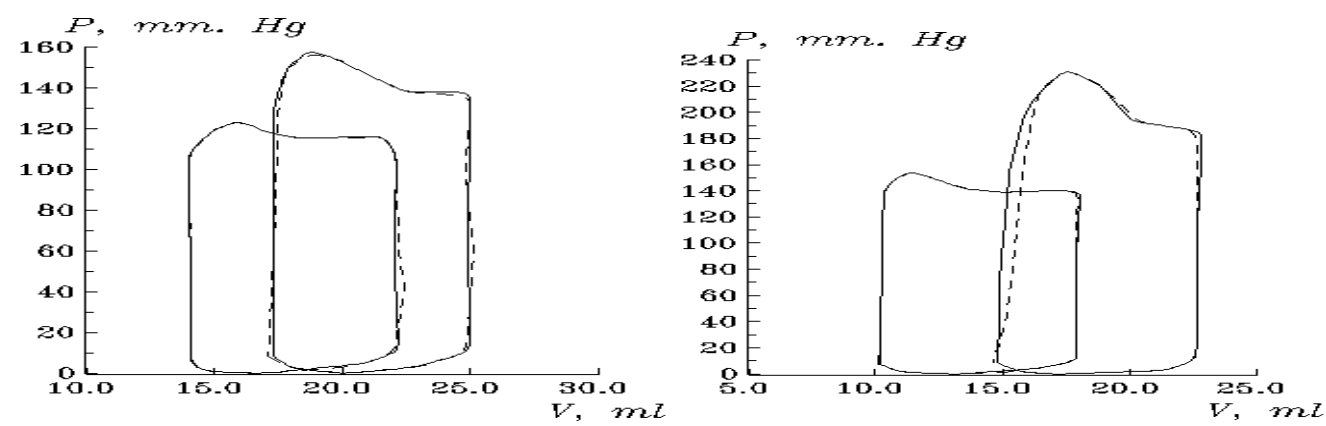
Comparisons between direct measurements of pressure in patients' left ventricle and results of modeling were executed in Kiev Institute of clinical surgery. Has appeared, that the difference between measured and calculated pressure in left ventricle did not surpass $10 \%$.

Conclusions: Is shown, that the combination of noninvasively examination and application of mathematical model permits noninvasively to determine a spectrum of biomechanical and hemodynamical functions and parameters of the cardiovascular, to decide problems of choice, forecasting of results and monitoring of treatment.

\title{
References
}

[1] Kantor B.Y.,Yabluchansky N.I.\&Martynenko A.V. Noninvasive diagnosis of biomechanical disturbances in the left heart, Naukova Dumka, 1992.

[2] Kantor B.Y., Yabluchansky N.I. \& Martynenko A.V. Interpretation of results from functional investigations of cardiovascular system, "Osnova" State Univetsity Publ., 1993.

[3]Nozawa T. et al. Eficiency of energy transfer from pressure-volume area to external mechanical work increases with contractile state and decreases with afterload in the left ventricle of the anesthetized closed-chest dog, Circulation, $1988,77,5,1116-1124$.

\section{Finite element analysis to quantify tissue prolapse within three designs of cardiovascular stents}

\author{
C. Lally ${ }^{\mathrm{a}}$, P.J. Prendergast ${ }^{\mathrm{a}}$, T.C. Lee Le, $^{\mathrm{a}, \mathrm{b}}$ D. Quinn ${ }^{\mathrm{c}}$ and F. Dolan ${ }^{\mathrm{c}}$ \\ ${ }^{a}$ Bioengineering Group, Dept. of Mechanical Eng., Trinity College, Dublin 2, Ireland \\ ${ }^{\mathrm{b}}$ Department of Anatomy, Royal College of Surgeons in Ireland, Dublin 2, Ireland \\ ${ }^{\mathrm{c}}$ Medtronic AVE, Parkmore Industrial Estate, Galway, Ireland
}

Introduction: Intravascular stents have been developed to maintain vessel patency after balloon angioplasty. Although, stents increase the arterial lumen size above that achieved by balloon angioplasty alone, in-stent restenosis still remains a major limitation to the late outcome of the procedure. It occurs in 20-50\% of stented vessels1. Tissue 'prolapse', i.e. draping of the tissue within the units of the stent, has been related to the progression of in-stent restenosis 2 . This study presents a quantification of tissue prolapse within three different stent designs (BeStent 2, Medtronic; NIROYAL, Boston Scientific; Velocity, Cordis ) to determine the optimum design to minimise restenosis.

Methods: To compare tissue prolapse within the BeStent 2, NIROYAL and Velocity stent, the geometry of a fully expanded repeatable stent unit of each stent design was determined using a coordinate measurement technique (Figure 1). Using a two dimensional approximation of the slightly curved stent unit geometry, finite element models of the arterial tissue within the stents were generated numerically using ANSYS (Release 5.5).

The material within the stent unit was modelled using a Mooney-Rivlin hyperelastic constitutive equation, which has been found to adequately describe the non-linear stress-strain relationship of elastic arterial tissue3.

Uniaxial and equibiaxial tensile tests were performed to determine the stress-strain relations of human femoral arteries and porcine aorta4, necessary to establish the specific hyperelastic constants for these biological materials. 
The thickness of the arterial tissue in the numerical models was taken as $0.5 \mathrm{~mm}$, based on the thickness of the human coronary artery wall. The models were restrained around the edges of the stent unit, simulating the stent wire.
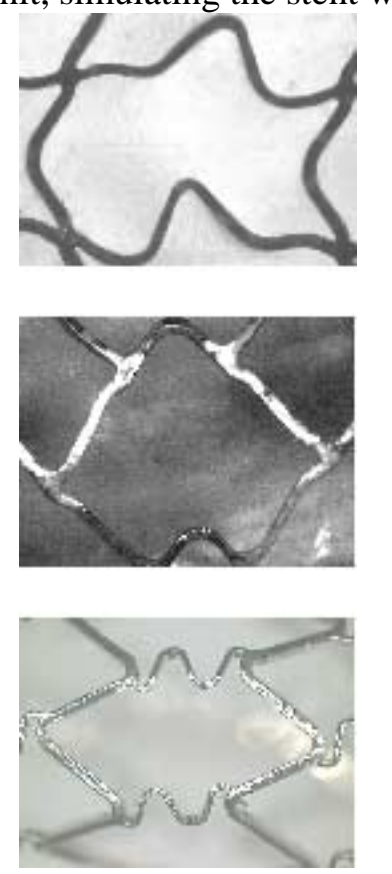

Fig. 1: Geometry of Stent Units; (a) BeStent2, (b) NIROYAL and (c) Velocity stent design

This was considered to adequately represent the arterial tissue within the stent unit, since the stent wire thickness $(0.1 \mathrm{~mm}$ approx.) is less than the thickness of the tissue.

A surface pressure of $450 \mathrm{mmHg}$ was applied. This surface pressure simulates the radial pressure on the stent from the retraction of the artery wall.

Results:The degree of tissue prolapse, i.e. the maximum 'draping' displacement of the tissue, was calculated. The arterial tissue properties have been defined as either those for porcine aorta or human femoral arterial tissue. The extent of tissue draping within the Velocity stent was found to be considerably greater than in the NIROYAL stent and the BeStent 2 design, with the latter showing the least draping of all the stent designs, see Figure 2.

Tissue prolapse was found to be considerably greater in the stent units where the tissue properties were described using the porcine aorta tissue properties compared to the human femoral artery. This is because porcine aorta is more elastic. It is postulated that the coronary artery, into which stents are implanted, would have similar properties to that of the human femoral artery.

Discussion: From this study, it is clear that a significantly greater degree of luminal tissue prolapse would occur within the Velocity and the NIROYAL stents compared with the BeStent 2 . However, the tissue properties used in this study are those for healthy arteries. It is therefore proposed to use the stress-strain relationship for restenotic tissue, obtained from uniaxial and equibiaxial tensile tests on the tissue, to describe the material properties of diseased tissue more accurately. Further investigations of tissue prolapse within a number of other stent designs will also be carried out to determine the 
optimum stent to limit late lumen loss in stents, resulting from tissue prolapse and the development of in-stent restenosis.

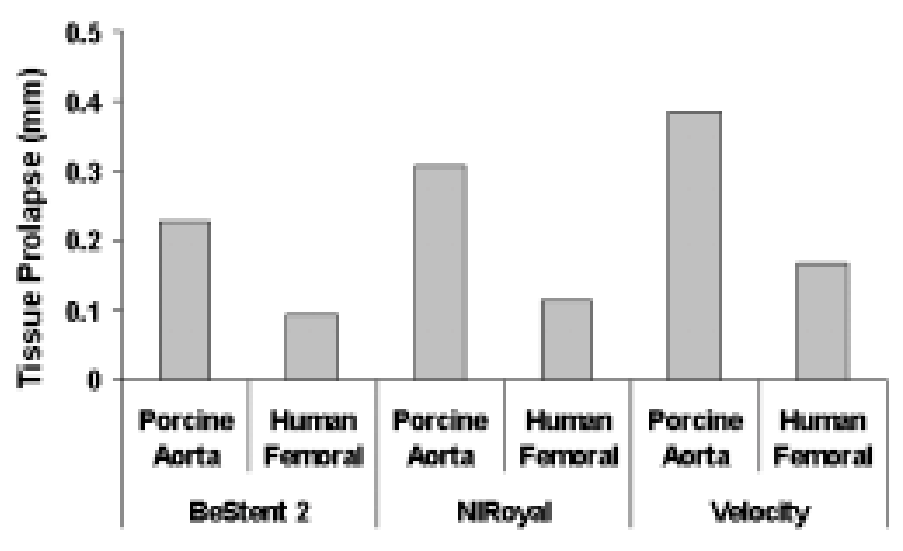

Fig. 2: Maximum Tissue Prolapse within the BeStent2, NIROYAL and Velocity Stents

\author{
References \\ [1] P.H. Grewe, T. Denke, A. Machraoui, J. Barmeyer, K.-M. Muller. J Am Coll Cardiol. 35: 157-163 (2000). \\ [2] C.K. Ponde, C. N. Aroney, P.T. McEniery, J.H.N. Bett. Cathet Cardiovasc Diagn. 40: 353-357 (1997). \\ [3] K. Hayashi, Y. Imai. J Biomech. 30: 573-579 (1997). \\ [4] S. Daly, P.J. Prendergast, F. Dolan, T.C. Lee. Proc. 12th European Society of Biomechanics, p. 242 (2000).
}

\title{
Application of neural networks to flexible solid state ion selective sensor arrays for the detection of myocardial ischemia
}

Mark Hyland, Norbert Pilz, James McLaughlin, Eric McAdams

The Northern Ireland Bioengineering Centre (NIBEC), University of Ulster at Jordanstown, Shore

Road, Newtownabbey, County Antrim, BT37 OQB, Northern Ireland

Tel: +44 (0) 2890366898

Extended Abstract: In Europe, tens of thousands of heart by-pass and heart transplants are performed each year. During the surgical procedure the heart is arrested and normal methods of determining if the heart is under stress (e.g. E.C.G.) cannot be used. Clinical experimentation has found that as the cell walls in the myocardium begin to collapse during Ischemia then the chemical processes occurring can be detected with chemical sensors (1). If such sensors have a fast response time (e.g. seconds) then the heart can be re-perfused and the Ischemia can be reversed. However, a second stage of Ischemia does occur when the chemical changes are irreversible and the heart is permanently damaged. The NIBEC group has developed a novel sensor array system, enabling a surgeon to be aware of the onset of Ischemia and when irreversible Ischemia is developing. Additionally, during organ transplant, a surgeon has little idea if an organ is viable when he gets it. Often, the decision to use the organ is made simply by a visual assessment and knowledge of the time lapsed since the organ was harvested. Obviously, sensor arrays like the ones developed at NIBEC will lead to increased and more effective use of transplanted organs. Knowledge of the chemistry occurring 
in the myocardium at all stages of the transplant is also expected to lead to more effective transplant procedures.

Arrays of ion selective sensors have been manufactured for the detection of $\mathrm{K}+, \mathrm{Na}+, \mathrm{Ca} 2+$, concentration, $\mathrm{pH}$, and tissue impedance and temperature. Solid-state ion selective sensors are developed using thin film technology supported by flexible polymer substrates. Photolithographic processing of the metallic and insulating layers facilitates freedom of sensor layout and allows for high density, small dimension sensors of around 10 microns to be manufactured. The sensor arrays exhibit minimal tissue damage, good conformity, and biocompatibility. Figure 1 shows the cross section of an ion selective electrode device.

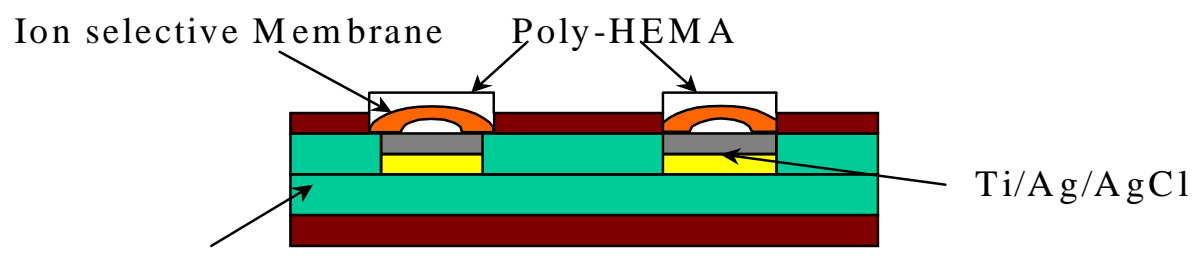

Polyimide film

Figure 1: Schematic cross section of an Ion selective electrode device (two sensors)

The sensor arrays mentioned above, when exposed to mixtures of ionic solutions exhibit good Nernstein responses $(54 \mathrm{mV} /$ decade $+/-5 \mathrm{mV} /$ decade) but show low selectivity and strong temperature dependence. To overcome these problems a Neural Network algorithm has been developed which uses the output of the whole array of sensors to classify and quantify the ionic concentrations with increased accuracy (2). In particular a multiple layer feed forward network structure in combination with a back propagation-learning algorithm is utilised (3). The Neural Network has been incorporated into a LabView( program from National Instruments for the immediate display (in real time) of ionic concentrations along with a history of the ion profile in the myocardial wall. Results are presented using the ion selective array, both in conjunction with the Neural Network algorithm and without it. It is found that the algorithm interprets the signals of sensor arrays into ion concentrations with increased accuracy of the measurement and negates the need for sensors with more refined selectivity characteristics. Table 1 shows the maximum error obtained for solutions with maximum concentrations of $80 \mathrm{mMol}$.

Table 1: Maximum error of the measured ion concentration

\begin{tabular}{llcccr} 
& $\mathrm{K}+$ & $\mathrm{Na}+$ & $\mathrm{H}+$ & \multicolumn{2}{c}{$\mathrm{Ca}^{2}+$} \\
RMS_error [Mol] & 0.00063 & & 0.00070 & 0.00102 & \\
0.00040 & & & & & \\
$\begin{array}{l}\text { max_error [Mol] } \\
0<\mathrm{c}<80 \mathrm{mMol}\end{array}$ & 0.00124 & & 0.001462 & 0.003799 & 0.000586
\end{tabular}

Although the implemented Neural Network shows good results, for medical applications the sensitivity must be optimised. Experiments are ongoing with the aim to investigate appropriate algorithms to limit the maximum error of each ion to $+/-0.5 \mathrm{mMol}$. 


\section{References}

[1] Errachid A., Ivorra A., Aguiló J., Villa R., Millán J., Zine N., Bausells J. urosensor, "New technology for multi-sensor silicon needle for biomedial applications" Copenhagen, DK, August 25-27, 2000

[2] HASHEM, S., KELlER, P. E., KOUZES, R. T., KANGAS, L. J.: 'Neural Network Based Data Analysis For Chemical Sensor Arrays', Proceedings of the SPIE, 1995, 2492 (5), pp. 33 - 40

[3] RALUCA-IONA, S., VAN STADEN, J. F., ABOUL-ENEIN, H. Y.: 'Electrochemical Sensor Arrays', Critical Reviews in Analytical Chemistry, 1999, 29 (2), pp. 133 - 153

\section{A completly magnetically levitated axial-flow blood pump: results from pre-clinical applications}

Johannes Müller ${ }^{\mathrm{a}}$, Yo-gou Weng ${ }^{\mathrm{a}}$, Peter Nüsser ${ }^{\mathrm{b}}$, Hans-Erhard Peters ${ }^{\mathrm{c}}$, Kurt Graichen ${ }^{\mathrm{b}}$, Andreas Arndt $^{\mathrm{b}}$, Werner Neumann ${ }^{\mathrm{b}}$, Georg Konstas ${ }^{\mathrm{d}}$ Jörg Müller $^{\mathrm{b}}$, Hendrik Heinze ${ }^{\mathrm{b}}$, *Jan Hoffmann ${ }^{\mathrm{a}}$, Tobias Merkel $^{\mathrm{b}}$, Conrad Kauffeldt ${ }^{\mathrm{b}}$, Ali Kilic ${ }^{\mathrm{b}}$, Peter Göttel ${ }^{\mathrm{b}}$, Frank Deus ${ }^{\mathrm{b}}$, Ines Goebel ${ }^{\mathrm{b}}$, Roland Hetzer ${ }^{\mathrm{a}}$ ${ }^{a}$ Deutsches Herzzentrum Berlin, Berlin, Germany

${ }^{\mathrm{b}}$ Berlin Heart AG, Berlin, Germany

${ }^{\mathrm{c}}$ Universität Rostock, Rostock, Germany

${ }^{\mathrm{d}}$ Wittenstein cyber motor GmbH, Igersheim, Germany

Introduction: Mechanical bearings in axial-flow pumps have inherent friction, which may sometimes cause thrombus formation. Therefore, we have developed an axial-flow pump as a left ventricular assist device (LVAD) with magnetic bearings for the impeller. The absence of friction and heat generating poles is a favourable condition for a low thrombogenecity and virtually unlimited durability.

Methods: Placement of both, the magnets for the motor and the bearings in the hub of the impeller avoided any restriction on the blade design. The overall characteristics of the pump are as follows: Power consumption at the operating point of $8.600 \mathrm{rpm}$ and $5 \mathrm{~L} / \mathrm{min}$ blood flow against $100 \mathrm{mmHg}$ pressure head across the pump is $8.5 \mathrm{~W}$ (including motor, bearings and electronics), pump output may be increased up to $7 \mathrm{~L} / \mathrm{min}$ against $150 \mathrm{mmHg}$, the weight of the pump is $200 \mathrm{~g}$, its outer diameter is $30 \mathrm{~mm}$, flow and pressure measurement is integrated in the pump housing. For the levitation of the impeller the pump is equipped with a radially passive and axially active magnet system. Computational fluid dynamic analysis (CFD) and experimental analysis of the pump's interior flow field were used to accomplish a design with an optimal flow pattern.

Results: Testing of hydraulic performance and in vitro and in vivo testing for hemolysis confirmed excellent properties in this respect. In in vitro experiments a NIH of $6 \mathrm{mg} / 100 \mathrm{~L}$ and $\mathrm{MIH}$ of $1 \mathrm{had}$ been achieved. In the first 2 animal experiments in sheep no increase of plasma free hemoglobin (PFH) was seen, however, LDH increased from 550 to $900 \mathrm{U} / \mathrm{L}$, but remained stable at this level. Liver enzymes and urinary excreted substances raised slightly.

Discussion: Despite of the levitating principle of the pump and its optimised fluid dynamic characteristic with the beneficial effect on the flow pattern and hemolysis, the size of the pump could be kept small. The overall test results of this axial-flow blood pump are very encouraging. They have to be confirmed in long-term animal tests before starting clinical trials.

\section{Experimental evaluation of the migration of aortic stent grafts}


Morris, $\mathrm{W}^{\mathrm{a}}$, Delassus, $\mathrm{P}^{\mathrm{b}}$, and McGloughlin, $\mathrm{T}$

${ }^{a}$ Biomedical Engineering Research Centre, Department of Mechanical \& Aeronautical Engineering, University of Limerick, Limerick, Ireland

${ }^{\mathrm{b}}$ Galway Mayo Institute of Technology, Dublin Road, Galway, Ireland

Introduction: Endovascular grafting is an alternative to the surgical repair method of treatment of Abdominal Aortic Aneurysm (AAA). This treatment is a less invasive method of treating these lifethreatening aneurysms. Secure proximal fixation of stent grafts for AAAs is pivotal to the long-term success of this procedure.

The purpose of this study was to assess the suitability of the various commercially available stent types for the treatment of AAAs. Stent graft migration is a recognized problem has been reported by several researchers [1]. The conditions needed to cause migration was achieved experimentally by a bench test system, that is capable of simulating pulsatile pressure and flow. Applying these conditions the resultant force was calculated for the bifurcated stent graft. The possibility of stent graft migration poses a serious threat to the long-term durability of the stent graft. The issues of wave reflections are addressed and are a recognized factor in increasing the proximal pressure. The values of the resultant forces are compared to tests carried out by other researchers who found the longitudinal force required to cause stent graft migration [2,3]. This comparison will help provide design guidelines for proximal stent anchorage systems and their capability of withstanding the pulsatile forces created in the vascular system.

Materials and Methods: A bench test facility was built to replicate the conditions of the cardiovascular system and the disease in question aortic aneurysms at the iliac bifurcation. A piston pump was built to simulate pulsating flow. Silicon aortic aneurysms were manufactured by the lost wax process. A valve with a varying spring stiffness and hence varying pressure was used to keep the aorta pressured similar to that in the body. Shape memory alloy smooth Z-shaped stents using the super elastic effect were manufactured and sewn onto bifurcated polyester grafts. Three different situations were tested in the bench test to find the conditions needed to cause stent graft migration.

Condition 1 - A bifurcated graft with only one stent at the proximal end.

Condition 2 - A bifurcated graft with one stent at each end i.e one at the proximal end and one each at both iliac ends.

Condition 3 - a fully stented structure.

Results: The results showed that for condition 1 and a pump setting of $72 \mathrm{rpm}$ (normal pulse rate), a pressure of $6848 \mathrm{~N} / \mathrm{m} 2(51 \mathrm{mmHg})$ was required to cause stent graft migration. This corresponds to a calculated maximum resultant force of $2.5 \mathrm{~N}$. The same settings that caused migration for Condition 1 caused stent graft migration for Condition 2. However, for condition 3 the stent graft remained in place for conditions far in excess of that experienced in the vascular system.

Discussion: As has been demonstrated by the physical test, one smooth stent at either end of a stent graft is not suitable for fixation. The resultant forces for conditions 1 and 2 are comparable to the results of $[2,3]$. For condition 2 buckling of the fabric was the problem. This proves that the fixation characteristics of smooth stent grafts are not capable of withstanding the pulsating forces generated in the vascular system. For condition (3) a fully stented structure was capable of withstanding a condition far in excess of that experienced in the vascular system. The results will help provide design guidelines for proximal stent anchorage systems. 


\title{
References
}

[1] EUROSTAR Data Registry Centre., July 2000. Laheij R., Van Marrewijk C., Buth J., Progress Report on the procedural and follow up results of 2955 patients who received stent graft treatment for infra-renal abdominal aortic aneurysms for a period of 60 months.

[2] Resch T., Malina M., Lindblad B., Malina J., Brunkwall J., Ivancev K., 2000. The impact of stent design on proximal stent-graft fixation in the Abdominal Aorta: an Experimental Study. Eur J Vasc Endovasc Surg 20. 190-195.

[3] Malina M., Lindblad B., Ivancev K., 1998. Will stents with hooks and barbs prevent stent-graft migration? J Endovasc Surg 5. 310-317.

\section{Session - Bone and Joint Biomechanics Room 2, Saturday 5 May, 16.15 - 17.30}

\section{Changing of the micro - mechanical properties of bone after $x$-ray irradiation (Proved by scanning acoustic microscopy and environmental scanning electron microscopy)}

\author{
I. Eckardt ${ }^{\mathrm{a}}$, S. Henning ${ }^{\mathrm{b}}$, F. Syrowatka ${ }^{\mathrm{c}}$, H.-J. Hein ${ }^{\mathrm{a}}$ \\ ${ }^{a} R G$ Biomechanics and Structure Research, Dept. of Orthopaedics, Str. d. OdF 4, D-06097 Halle, \\ Germany \\ ${ }^{\mathrm{b}}$ Dept. of Materials Science \\ ${ }^{\mathrm{c}}$ Center of Materials Science; Martin-Luther-University, Halle-Wittenberg, Germany
}

Introduction: Aim of the work is to investigate the influence of ionizing irradiation on the structure and mechanical properties of human cortical bone. Samples were irradiated with a series of x-ray doses. Scanning acoustic microscopy (SAM) and environmental scanning electron microscopy (ESEM) is applied on the characterization of changes in morphology and micro-mechanical behaviour. First results will be presented.

Materials and Methods: A specimen from a human cortical femur (71 old, male) was embedded native in PMMA, cut in a $60^{\circ}$ angle (respecting to the bone longitudinal axis) and polished.

The surface was first investigated by scanning acoustic microscopy $[1,2]$ using a KSI SAM2000 and a $400 \mathrm{MHz}$ lens scanning a range of $1 \mathrm{~mm} 2$. Since it is impossible to produce a completely flat bone surface by polishing for each area a series of pictures with decreasing surface to lens distances was taken leading to the information of the surface distribution of the acoustic impedance $Z=$ ? $\bullet v 1$ (?material density, vl-longitudinal ultrasound velocity) and a topography information too [3,4].

After SAM measurements the sample was irradiated with $125 \mathrm{keV}$ photons three times using doses of 1,3 and 3 Gray again.

The morphology and the topography of irradiated and non-irradiated samples were imaged using a PHILIPS XL30 ESEM-FEG environmental scanning electron microscope. One of the main advantages of the new „environmental" technique is that no carbon or metal coating is required as for conventional SEM. The samples do not undergo excessive drying as in preparation steps necessary for high vacuum conditions. These features allow to carry out sequences of radiation exposure/SAM/ESEM/radiation exposure, and so on. The instrument was operated at an accelerating voltage $15 \mathrm{kV}$ for secondary electron (SE, topography) and $30 \mathrm{kV}$ for backscattered electron (BSE) material contrast images, respectively [5]. Best results for BSE micrographs were achieved in nitrogen 
(N2) at a pressure of 2.4 Torr. EDX spectra were recorded at regions showing significant differences in their height profile or in their BSE intensity.

Results and Discussion: Eight SAM 1mm2 measurements through the whole corticalis were done before radiation. We selected a region near the spongiosa to represent the irradiation effect on the micromechanical properties.

Before irradiation there is a strong contrast between older osteonal regions and the younger one, the lower hardness of the last results in a lower intensity. Already after 1 Gray irradiation the reflection of the ultrasound wave from the surface is suppressed. The highest intensity of the signal (producing the resulting picture) comes from a region some microns below the surface; the image is similarly to a defocused case and shows a reversion of the contrast in some regions. From this unusual image process we conclude that the surface structure must have changed (probably roughened) during irradiation. There is no qualitative change in SAM pictures by increasing of the irradiation dose up to 7 Gray.

The radiation damages of the surface structure can be removed by polishing, but SAM images show a decrease of the contrast indicating an embrittlement of the softer regions.

Images of the irradiated samples recorded in the SE mode exhibit a sharp step in the height profile between two adjacent osteons. The region belonging to the osteon in the upper part of the image is above the structures of the osteon in the lower part. From the analysis of a series of stereographical images a step height of $(2 \mu \mathrm{m}$ was calculated. In the osteonal centre, a haversian channel filled with embedding medium (PMMA) reaches out of the bone surface. An interlamellar crack, partially bridged by fibrils, is detected. The same region was detected in the BSE material contrast mode. The differences in the grey levels in BSE images can be interpreted as local variations in bone mineral density [7]. The upper "sheet" of bone appears in lighter shades of grey representing a higher mineral (hydroxy apatite - HAp) content. These results lead to the conclusion that layers of bone material suffer embrittlement, and the process depends on the chemical composition (mineral content).

The differences in height produced by polishing seems to be lower then in the fresh irradiated case. The cracks have grown. Since it is known that specimen preparation techniques associated with hydration and dehydration of the samples introduce microcracks [6], this increase is an additional indication for embrittlement.

Energy dispersive $\mathrm{x}$-ray microanalysis was used to analyse the mineral composition at two spots corresponding to the upper region and the lower region of the surface topography of irradiated bone. A higher content of Calcium (Ca) and Phosphor (P) is detected (compared to the height of the oxygen peak) on the upper surface level compared to the lower surface level.

These results prove that the differences in the BSE grey levels are due to differences in Ca and $\mathrm{P}$ (HAp) concentration.

\section{References}

[1] [1] Briggs, A.: Acoustic Microscopy, Oxford 1992

[2] [2] Yu, Z. and Boseck, S.: Scanning acoustic microscopy and its applications to material characterization; Reviews Of Modern Physics, 67 (1995) 863

[3] [3] Eckardt, I. and Hein, H.-J.: Quantitative measurements of the mechanical properties of human bone tissues by SAM; Abstracts ACAS'99 (1999) 18

[4] [4] Raum, K. et al.: Quantitative Ultraschallmikroskopie zur Bestimmung der akustischen Impedanz von kortikalem Knochengewebe; Z. Med Phys., 9 (1999) 246

[5] [5] Schaffler, M.B. et al.: Examination of Compact Bone Microdamage Using Back-Scattered Electron Microscopy; Bone 15(5)(1994), 483

[6] [6] Roschger, P. et al.: Formation of Ultracracks in Methacrylate-Embedded Undecalcified Bone Samples by Exposure to Aqueous Solutions; Cells and Materials 3(4) (1993), 361 
[7] [7] Roschger, P. et al.: A New Scanning Electron Microscopy Approach to the Quantification of Bone Mineral Distribution: Backscattered Electron Image Grey-Levels Correlated to Calcium K alpha-Line Intensities; Scanning Microscopy 9(1) (1995), 75

\title{
Microcrack accumulation during fatigue testing of compact bone
}

\author{
F.J. O'Brien, D. Taylor, T.C.Lee \\ Dept. of Anatomy, Royal College of Surgeons in Ireland and \\ Dept. of Mechanical Engineering, Trinity College, Dublin
}

Introduction: Fatigue damage in bone occurs in the form of microdamage. This microdamage contributes to the formation of stress fractures and acts as a stimulus for bone remodelling [1]. Microdamage is also known to accumulate with increasing age leading to the formation of fragility fractures. A technique has been developed [2,3], which allows microcrack growth to be monitored during the course of a mechanical fatigue test by the application of a series of fluorescent chelating agents. These are as effective as the standard method, basic fuchsin, in identifying microcracks but are also site specific as they chelate calcium ions lining the crack walls. Each agent fluoresces a different colour under UV light and so individual agents can be distinguished when viewed using UV epifluorescence microscopy. By applying agents before and at different intervals during a mechanical test, it was proposed to label microcrack development in order to look at the process by which microcracks initiate, propagate, interact with the bone's microstructure and ultimately bring about failure.

Methods: Specimens were taken from fresh bovine tibiae and fatigue tested in cyclic compression at a stress range of $80 \mathrm{MPa}$. The specimens were stained before testing with alizarin (which has the greatest affinity for calcium) in order to label pre-existing damage. They were tested in another agent, xylenol orange for the first 10,000 cycles. This agent was then replaced with calcein to 50,000 cycles and finally calcein blue between 50,000 cycles and failure. The gauge length was removed from each specimen and divided into 2 equal parts. One part was sectioned in the transverse direction using a diamond saw (Streurs Minitom) while the other half was sectioned longitudinally. Sections were analysed for labelled microcracks using UV epifluorescence microscopy. The results were tabulated in the form of: no. of cracks labelled with each agent occurring per section, crack numerical density (Cr.Dn. no./mm2), crack surface density (total $\mu \mathrm{m}$ of crack/ mm2 ) and mean crack length $($ Cr.Le. $\mu \mathrm{m})$. Cracks in transverse sections were also described in terms of location i.e: osteonal, where they entered a cement line or concentric lamellae of a secondary osteon, or interstitial, where they were located in interstitial bone between osteons. Unpaired t-tests with a probability value of $95 \%$ $(\mathrm{p}<0.05)$ were used to analyse the data.

Results: The chelating agents allowed a clear distinction to be made between pre-existing microcracks, microcracks formed in the first part of the test (up to 50000 cycles) and microcracks formed in the remainder of the test (up to failure). Only $6 \%$ of microcracks were preexisting and these did not propagate during testing. A greater proportion of microcracks were found in transverse than in longitudinal sections $(\mathrm{p}<0.05)$.

Fig. 1 illustrates the pattern of microcrack accumulation during the course of a test. Similar results were found for both transverse and longitudinal sections. Microcracks were shown to develop rapidly during the first 10,000 cycles, but no significant increase took place between 10,000 cycles and 50,000 
cycles. A further increase in microcrack- density then took place between 50,000 cycles and failure with the average Nf being 90,190 (S.D. 21,100) cycles to failure.

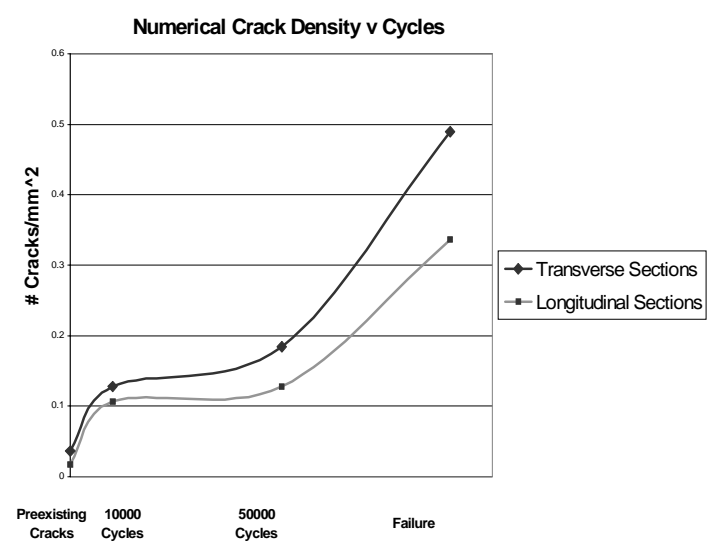

Fig. 1: The process of microcrack accumulation during testing.

Discussion: Microcracks were found to accumulate early on in a specimen's life but the rate of accumulation then slows down until an increased rate of accumulation in the period prior to failure. Bone microstructure greatly influenced microcrack growth with the vast majority of microcracks being found in interstitial bone between Haversian systems. Microcracks have been shown to grow in length primarily in the longitudinal direction, parallel to the longitudinal axis of the bone. Numerous authors have mentioned the possibility of a microstructural barrier existing in bone. These results support this concept whereby the morphology of osteonal bone appears to provide an abundance of crack initiation sites but its structure prevents or slows any significant crack growth. The work provides new data on the behaviour of microcracks in compact bone.

\section{Acknowledgements}

This work was funded by Health Research Board of Ireland, Cappagh Hospital Trust, Dublin and the Research Committee of the Royal College of Surgeons in Ireland.

\section{References}

[1] M.B.Schaffler, K. Choi, C. Milgrom, Bone. 17: 521-525 (1995).

[2] T.C. Lee, T.L. Arthur, L.J. Gibson, W.C. Hayes, J. Ortho. Res., 18: 322-325 (2000).

[3] T.C. Lee, F.J. O'Brien, D. Taylor, Int. J. Fat. 22: 847-853 (2000).

Hardness and elasticity modulus of bone compartments of the femur head measured by microindentation

R. Wolff ${ }^{\mathrm{a}}$, W. Fränzel ${ }^{\mathrm{b}}$, P. Grau ${ }^{\mathrm{b}}$, H.-J. Hein ${ }^{\mathrm{c}}$

${ }^{a}$ Technical College, Köthen/Anhalt, Dept. of Medical Techniques

${ }^{\mathrm{b}}$ Martin-Luther-University Halle-Wittenberg, Dept. of Physics 
${ }^{\mathrm{c}}$ Martin-Luther-University Halle-Wittenberg, Med. Faculty,Dept.of Orthopaedics, R.G. Biomechanics \& Structure Research

Introduction: Depending on loading and ageing of the femur, the trabecels in the head have different hard-ness and elasticity values, respectively. This is due to the modelling and remodelling of bone. Especially in the case of diseases, it may be assumed that the elastomechanical values are different from the norm. In such cases, the changing of these parameters is very sensitive to detection. Other authors have shown that the values measured by nanoindentation (Rho et al.,[1]), as well as by acoustic microscopy (Katz et al., [2]), (Hein et al.,[3] ) yield a slight difference in hardness and elasticity, respectively. These differences depend on the kind of method used, the site of measurement in the micro-region and the kind of bone, of course.

The trabecels are between at least $50 \mu \mathrm{m}$ and more than $200 \mu \mathrm{m}$. So, it is justifiable to apply recording microindentation to characterize the elastomechanical parameters of the trabecels. The results achieved are comparable with other well-established methods in this field. The main advantage is that microindentation is easier to use and much cheaper.

Material and Methods: The material under investigation is taken from the human femur head. A $100 \mu \mathrm{m}$-thick slice is cut from the latter. The slice is prepared and embedded in PMMA using conventional meth-ods. Finally, the specimen is ground and polished. In order to achieve a unique distribution of indentations, the specimen is divided up into regions of interest. It is used the recording microindentation with a Vickers indenter. Using this

device it is possible to continuously measure the load and penetration depth, and thus obtain a load penetration depth - curve. This curve shows all the information on the elas-tic and plastic behavior of the region investigated. Oliver and Pharr [4] developed a method to determine the elasticity modulus from the unloading curve. For the determination of the elasticity modulus, the contact depth is important. This term de-scribes the depth of indentation at which the indenter is completely into contact with the material.

Results and Discussion: To appreciate the influence of the embedding material on the results, the hardness of the PMMA and the bone is measured independently.

A comparison of the hardness values yields a clear difference between PMMA and the bone material. The hardness of the bone is nearly three times higher than that of PMMA, independ-ent of several maximum loads Fmax. Thus, the influence of the embedding material on the re-sults of the bone is negligible. All further measurements were carried out with Fmax =0,2 N.

We found that where there are fewer trabecel, the individual trabecel are harder than in other regions where there are more trabecel. The hardness values of the femur head are for trabecels between $460 \mathrm{MPa}$ and $525 \mathrm{MPa}$ depending on the place and for corticalis between $445 \mathrm{MPa}$ and 570 $\mathrm{MPa}$.

The maximum degree of hardness was to be found in the corticalis where there was a maximum load. In both trabecel regions, the hardness is significantly lower, because the loading is distributed over a larger volume in the region. These results are in keeping with our practical experience. For many reasons, it is interesting to determine the elasticity modulus from the indentation. It is only possible to determine this by continuously registering the data for the test force and indentation depth. From the character of the unloading curve, elasticity moduli of the bone materials may be determined. We deter-mined the elasticity moduli for the femur head. The elasticity moduli of the femur head are for trabecels between 13.5 and 17.5 GPa depending on the place and for corticalis between $12 \mathrm{GPa}$ and 15.7 GPa. 
A comparison of the hardness and the elasticity modulus of the region measured in femur head clearly shows the differences.

As an example, we determined the plastic energy consumed by the deformation of the material. Comparing the results with those of the hardness, we noticed that where the hardness has a maximum, the plastic energy has, in general, a minimum. There is no significant relation to the elasticity modulus.

As expected, the plastic energy for steel is much lower than that for bone or polystyrene. On the other hand, the values for corticalis and trabecels are nearly the same, and are comparable to those of polystyrene. The energy calculated can be used as a material constant from the point of view of the mechanical elasticity. The energy values depend on direction of structure which we measure.

\title{
References
}

[1] Rho, Y., Tsui, Y., Pharr, G.M., 1997. Elastic properties of human cortical and trabecular la-mellar bone measured by nanoindentation. Biomaterials $18,1325-1330$.

[2] Katz, J.L. and Meunier, A., 1993. Scanning acoustic microscope studies of the elastic proper-ties of osteons and osteon lamellae. Journal of Biomechanical Engineering 115, 543-548

[3] Hein, H.-J., Czurratis, P., Schroth, D., Bernstein, A.,1995. A comparative study of the appli-cation of scanning acoustic microscopy and confocal laser scanning microscopy to the structural assessment of human bones. Annals of Anatomy $177,427-430$.

[4] Oliver, W. C., Pharr, G. M., 1992. An improved technique for determining hardness and elas-tic modulus using load and displacement sensing indentation experiments. Journal of Materials Research 7, 1564 - 1583.

\section{Multiaxial fatigue of acrylic bone cement}

\author{
B.P. Murphy, P.J. Prendergast \\ Department of Mechanical Engineering, Trinity College, Dublin 2, Ireland
}

Introduction: Acrylic bone cement (PMMA) is subject to complex cyclic multiaxial stress states in vivo (Prendergast et al., 1989). However, there is no data in the literature to confirm how the multiaxial nature of the stress affects the fatigue life of acrylic bone cement; all fatigue data available at the moment is for a uniaxial stress. Stresses predicted in the cement mantle have been shown to be below the fatigue limit of bone cement by a factor of three (Prendergast et al., 1989), yet many retrieval studies point to fatigue failure as the predominant mode of damage accumulation in vivo (Topoleski et al., 1990). The question arises: "Are multiaxial stresses the cause of rapid fatigue failure in acrylic bone cement?"

Methods: Hollow cylindrical vacuum-mixed bone cement specimens were prepared using Cemex(c) Rx bone cement (Tecres, Verona, Italy). Vacuum mixing was carried out using the Optivac system (Scandimed A.B., Sjöbo, Sweden). All specimens were prepared at $22^{\circ} \mathrm{C}\left( \pm 1^{\circ} \mathrm{C}\right)$. The specimens were stored in a water bath at $37^{\circ} \mathrm{C}$ for a minimum of 2 weeks prior to testing. During the tests the specimens were submerged in a water bath and maintained at $37^{\circ} \mathrm{C}$, see Fig. 1.

The multiaxial stress state was created as follows: a constant pressure was applied internally to the specimen producing a tensile hoop stress and a compressive radial stress; simultaneously an axial stress was applied to the specimen by the actuator of the materials testing machine. Five different loading situations were applied to the specimen: two control groups with a uniaxial stress of either 11 $\mathrm{MPa}$ or $15 \mathrm{MPa}$, and three multiaxial stress states: $11 \mathrm{MPa}$ axial and hoop stress; $11 \mathrm{MPa}$ axial stress 
and 16.5 MPa hoop stress; 15 MPa axial and hoop stress. There were six specimens in each group

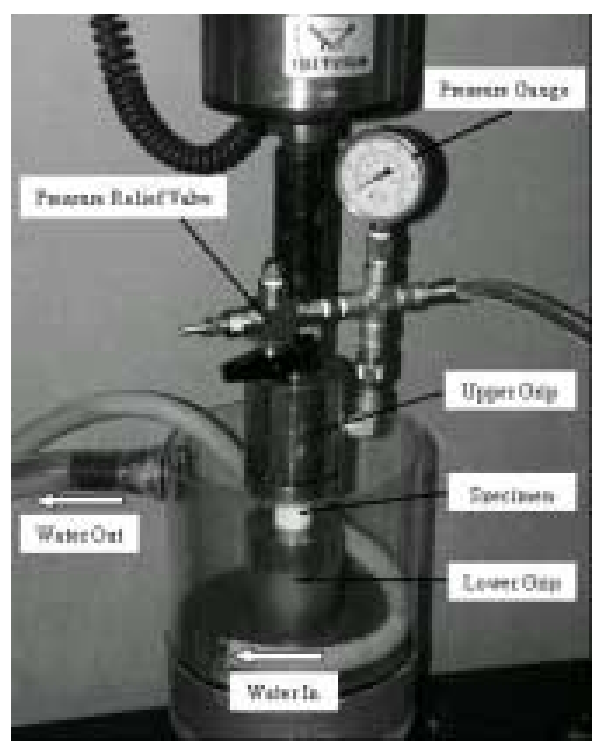

Figure 1 Schematic of the testing set-up.

Results: Results show that the application of a multiaxial stress state to acrylic bone cement can reduce the average fatigue life. At $15 \mathrm{MPa}$ the average fatigue life reduces to $40 \%$ of the uniaxial fatigue life when an equal hoop stress is applied. At a uniaxial stress of $11 \mathrm{MPa}$, a hoop stress of 16.5 MPa reduces the fatigue life to $30 \%$ of the uniaxial value. However an anomalous result is found at 11 $\mathrm{MPa}$ axial and hoop whereby an increase in fatigue life of $16 \%$ occurs, see (Table 1 for values). The significance value shows that no difference between the two data sets exists, this can be seen clearly when the distribution of fatigue strength is plotted using the two-parameter Weibull distribution, see Fig. 2. The distribution of fatigue strength for the higher hoop stress levels is consistently more variable that the control specimens and the group tested at the lower hoop stress level (see Fig 2).

Table 1 Summary fatigue results and significance values (Mann-Whitney directional test) comparing the control set to the pressurised groups.

\begin{tabular}{lrc}
\hline Axial 15 MPa & $\begin{array}{c}\text { Mean } \mathrm{N}_{\mathrm{f}} \\
(\mathrm{SD})\end{array}$ & $p$ value \\
\hline Hoop $=0$ & 68,957 & \\
& $( \pm 89,764)$ & \\
Hoop $=15 \mathrm{MPa}$ & 28,757 & 0.09 \\
& $( \pm 53,797)$ & \\
& & \\
\hline
\end{tabular}

(a)

\begin{tabular}{lrc}
\hline Axial 11 MPa & \multicolumn{1}{c}{$\begin{array}{c}\text { Mean } \mathrm{N}_{\mathrm{f}} \\
(\mathrm{SD})\end{array}$} & $p$ value \\
\hline Hoop $=0$ & 545,135 & \\
& $( \pm 635,276)$ & \\
Hoop $=11 \mathrm{MPa}$ & 646,062 & 0.41 \\
& $( \pm 437,963)$ & \\
Hoop $=16.5 \mathrm{MPa}$ & 159,302 & 0.06 \\
& $( \pm 177,326)$ & \\
\hline
\end{tabular}

(b) 


\section{$-\quad$ Control}

Figure 2 (a) Two-parameter Weibull distributions for $11 \mathrm{MPa}$ axial stress and (b) $15 \mathrm{MPa}$ axial stress.

Discussion and Conclusions: This study shows the profound effect of off-axis stress on the fatigue behaviour of acrylic bone cement. Average fatigue life is reduced by a factor of two and is statistically significant $(\mathrm{p}<0.1)$ for two of the multiaxial stress states. Typically, failure occurred by a fatigue crack growing perpendicular to the axial stress, although at the higher hoop stresses failure sometimes occurred by cracks growing perpendicular to the hoop stress. The increase in variability at the higher hoop stress levels may be caused by the effect of a second failure stress, i.e. the hoop stress, this stress now contributes more to the failure of the specimen thereby adding a further variable to fatigue failure.
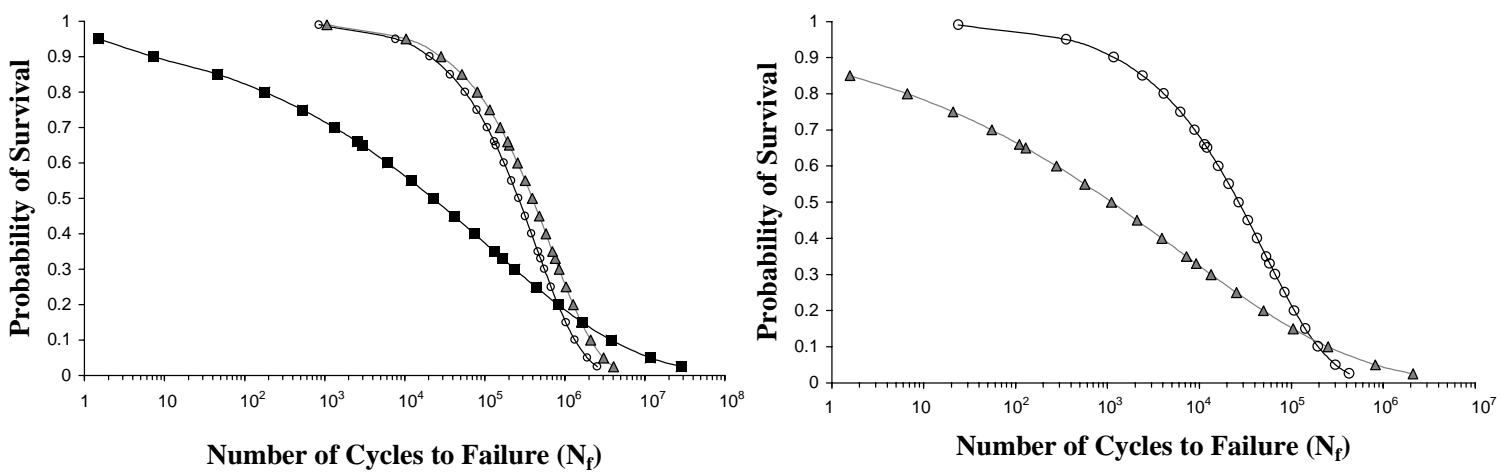

This study shows that an off-axis stress can affect the fatigue strength of acrylic bone cement; moreover, an increase in variation of fatigue strength occurs when multiaxial stresses are introduced; this variation is greater than that quantified previously by the authors for uniaxial stress (Murphy and Prendergast, 2000).

\section{References}

[1] Murphy B.P., Prendergast P.J., (2000), Int J Fat, 22, 885-864

[2] Prendergast, P.J., Monaghan, J., Taylor, D., (1989), Clin Mater, 4: 361-376

[3] Topoleski, L.D.T., Ducheyne, P., Cuckler, J.M., (1990), J Biomed Mater Res, 24, 135-154

\section{Reconstruction of zygomas with medical image based, custom made PMMA implants}

G. Jans ${ }^{\mathrm{a}}$, J. Vander Sloten ${ }^{\mathrm{a}}$, R.Gobin ${ }^{\mathrm{a}}$, G. Van der Perre ${ }^{\mathrm{a}}$, M. Mommaerts ${ }^{\mathrm{b}}$, K. Wouters ${ }^{\mathrm{c}}$

${ }^{a}$ Katholieke Universiteit Leuven, Division of Biomechanics and Engineering Design, Belgium

${ }^{\mathrm{b}}$ General Hospital St Jan Bruges, Division of Maxillo-Facial Surgery, Belgium

${ }^{\mathrm{c}}$ Materialise N.V., Belgium

Introduction: This paper reports on a clinical case of a 33 year old woman who had both zygomas removed because a disorder of the thyroid gland induced a massive growth of fat in the eye sockets. Figure 1 shows a CT-reconstruction of the skull. The removal of these bones reduced drastically the quality of life of the patient. The distal region around the eyes had collapsed and gave the face an unnatural look. Because of this, the patient remained in house most of the time, afraid of showing her face in public. 


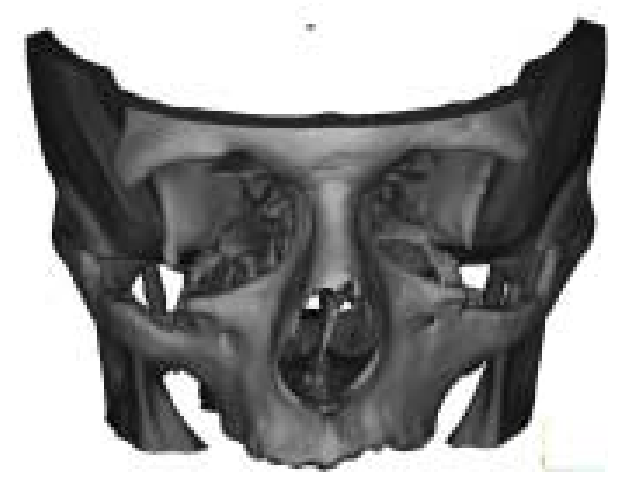

Figure 1: 3D-reconstruction of préop-CT's

The surgeon chose to insert personalised implants that would approximate the original zygomas as close as possible and at the same time reconstruct the spenoidale bone in a way that would enlarge the eye sockets sufficiently. Since no information on the shape of the original zygomas was available, the design of these implants had to be based on an extrapolation of image data regarding the remainder of the skull bones.

The implants were made out of polymethyl methacrylate. This is a strong material that can easily be casted and is cheap to use.

Materials and methods: After segmentation of the CT-data, the curves outlining the bony parts of the skull were constructed and transferred into CAD software. Simultaneously, a 3D-reconstruction of the skull was calculated and exported in STL-format. This makes the visualisation of the skull in CAD software possible.

Since both zygomas were missing, techniques like mirroring could not be used. There were also no CT-data available from other healthy persons that had a zygoma shape similar to one of the patient.

To facilitate the design, a new curve editor was developed and added to the CAD software. This editor makes it possible to view 3 or 5 consecutive CT-slices and adapt the intersection curves of the implant and the slice in the middle using the other slices as reference. The 3D-model was constructed by fitting a NURBS-surface through newly designed curves and converting it into a solid model. The model was then visualised together with the STL-reconstruction of the skull. By doing so, the design and fit of the new implants could be checked by the surgeon.

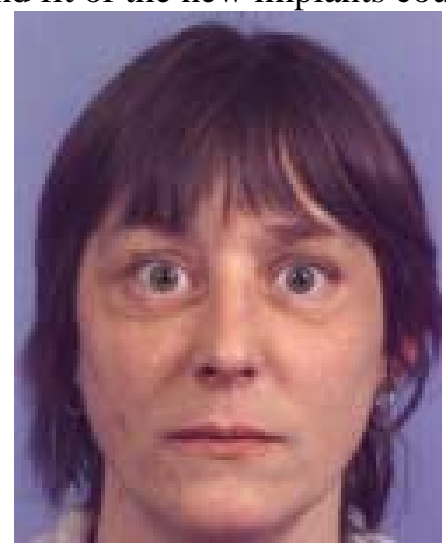

Figure 2: Picture after operation 
After final approval of the surgeon, the solid models were converted into STL-format and stereolithographic models were constructed. These were used to create silicone moulds in which the PMMA implants were casted.

The implants were inserted through a small incision under the eyes.

Results: The implants were generated in the CAD-software in collaboration with the surgeon. Communication took place over the internet using the commercial software "Communicator". This enables the users to view and manipulate a 3D-reconstruction of an STL-file simultaneously in different locations. It is also possible to share graphical remarks online.

The implants were designed and produced with a small overlap with the existing bone. This was done to give the surgeon the opportunity to take part of the remaining bone away if this would be necessary. Silicone moulds were made based upon stereolithographic reproductions of the computer models. Casting of the implants in PMMA was done in the hospital by people of the dental department.

The implants were inserted into a small incision that was made under the eyes, and not, as previously planned, by making an ear to ear incision and moving the skin forward over the eyes. The limited view made it difficult to estimate the amount of overlap that had to be removed and some chipping and fitting was necessary during the operation.

The patient was extremely happy with the result. The implants gave the face its natural look back. One implant was inserted a little bit oblique, but this showed only on CT and not on the outside of the face. Figure 2 shows a picture of the patient with the implants in place.

Discussion: The ideal implant would be made from fresh autogenous tissue. The additional procedure necessary to harvest the bone and the difficulty to shape it make it difficult to use this technique in such an aesthetically important position. Other materials that could be used include titanium. The cost and again the difficulty to establish the correct shape made the authors renounce this material in favour of PMMA. This material is strong, well tolerated by the human body and very easy to cast and therefore the best option to use for these implants. 